\title{
Spontaneous decay of resonant energy levels for molecules with moving nuclei
}

\author{
Stefan Teufel and Jakob Wachsmuth \\ Mathematisches Institut der Universität Tübingen \\ Auf der Morgenstelle 10, 72076 Tübingen, Germany
}

September 2, 2011

\begin{abstract}
We consider the Pauli-Fierz Hamiltonian with dynamical nuclei and investigate the transitions between the resonant electronic energy levels under the assumption that there are no free photons in the beginning. Coupling the limits of small fine structure constant and of heavy nuclei allows us to prove the validity of the BornOppenheimer approximation at leading order and to provide a simple formula for the rate of spontaneous decay.
\end{abstract}

\section{Contents}

1 Introduction 2

1.1 Electronic resonances for fixed nuclei . . . . . . . . . . . . . 6 6

1.2 Dynamical nuclei without coupling to the field . . . . . . . . . . . . 7

1.3 Dynamical nuclei with coupling to the field . . . . . . . . . . . . . . . 10

2 The main results 12

2.1 Born-Oppenheimer approximation with field . . . . . . . . . . . 13

2.2 Superadiabatic subspaces . . . . . . . . . . . . . . . . . 15

2.3 Spontaneous emission: transition operator . . . . . . . . . . . 16

2.4 Spontaneous emission: rate of decay . . . . . . . . . . . . . . . . . 22

3 Proofs of the main propositions

3.1 Proof of Proposition 1 . . . . . . . . . . . . . . . . . . . . . . 29

3.2 Proof of Proposition $2 \ldots \ldots \ldots \ldots \ldots$

3.3 Proof of Propositions $3 \& 4$. . . . . . . . . . . . . . . 35

4 Proofs of Lemmas 40 


\section{Introduction}

In the quantum mechanical description of atoms and molecules one usually neglects the coupling to the radiation field and thus the possibility of emission or absorption of photons. The charged nuclei and electrons only interact via the static Coulomb interaction. Still the predictions for the spectra of atoms and molecules are in very good agreement with experimental data usually gathered through interaction with light. Also the predictions for the dynamical behavior of molecules agree with the motion observed e.g. in chemical reactions. The reason for the good agreement lies in the smallness of the fine structure constant $\alpha \approx \frac{1}{137}$ that determines the strength of the coupling to the radiation field.

It is by now well understood even on a mathematical level how the coupling to the quantized radiation field changes the spectrum of the Hamiltonian operator describing an atom or a static molecule, e.g. [BFS, $\mathrm{HHH}$, AFFS, Fa, HaSe]. The quantum mechanical eigenstates become resonances, with energies close to the original eigenvalues, that decay nearly exponentially with a rate that can be computed perturbatively.

The quantum mechanical understanding of the dynamics of molecules is based on the Born-Oppenheimer approximation. Roughly speaking one assumes that if the electrons are initially in a certain eigenstate relative to the nuclear positions (e.g. in the ground state), they will remain in the "same" eigenstate relative to the nuclear positions even when the latter change. The electronic state is "slaved" in this sense, but by energy conservation the electronic energy level serves as an effective potential for the motion of the nuclei. The validity of the approximation was proved in various versions $\mathrm{HaJ}_{1}$, $\mathrm{MaSo}_{1}, \mathrm{SpTe}_{2} \mathrm{PST}_{2}, \mathrm{MaSo}_{2}$. It is an adiabatic approximation relying on the fact that due to their large mass the nuclei move slowly compared to the lighter electrons. While transitions between different electronic levels (so-called non-adiabatic transitions) are possible even without coupling to the radiation field, the probabilities for such transitions are usually exponentially small in the adiabatic parameter and thus negligible.

The content of this work is a mathematical analysis of molecular dynamics with the coupling to the quantized radiation field taken into account. Our first result is the validity of the Born-Oppenheimer approximation at leading order. This is of course expected, since the validity of the Born-Oppenheimer approximation has been confirmed experimentally in countless situations. Again the reason is the smallness of $\alpha$ which leads to small decay rates on the time scale set by the nuclear motion. This result is a rather straightforward consequence of combining the known quantum mechanical results on the Born-Oppenheimer approximation with standard time-dependent perturbation theory.

The main mathematical and physical problem solved in this paper is the determination of the rates of spontaneous emission for dynamical molecules, i.e. for situations where the nuclei undergo a nontrivial dynamics. Let us briefly discuss an example where these rates are relevant. In Figure 1 some electronic energy levels for a di-atomic molecule are schematically plotted as a function of the nuclear separation $R$. The ground state energy actually behaves like $R^{-6}$ for large $R$ and thus leads to a rather small attractive force for separated atoms in the ground state, the so-called van-der-Waals force. One strategy to accelerate the production of dimers is to excite one of the atoms, so that the molecular system is in the first excited state that behaves like $R^{-3}$ and thus leads 
to a stronger attractive force. Once the nuclei come close, the system goes either into the ground state by spontaneous emission of a photon or the nuclei will only scatter and separate again. One is thus interested in the probability for spontaneous emission within a finite time interval while the nuclei are sufficiently close. However, this probability is not governed by a fixed decay rate since the electronic state and thus the lifetime of the resonance changes with the location of the nuclei. In particular no exponential decay law can be expected.
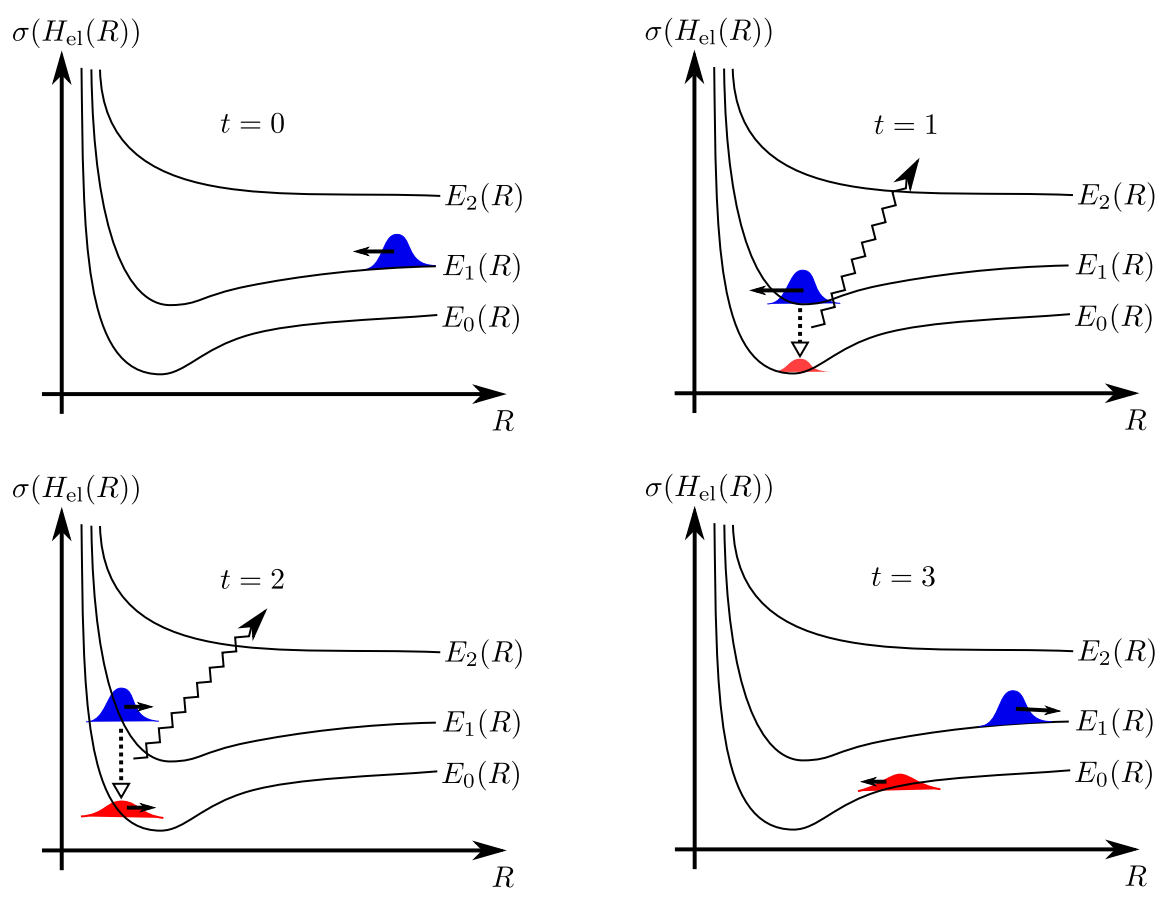

Figure 1: While moving in the electronic surface $E_{1}$, there is a configuration dependent probability for a transition to the ground state surface $E_{0}$ through spontaneous emission of a photon.

Our main result is an explicit time-dependent formula for the probability of spontaneous decay of a dynamical molecule through emission of a photon, for finite times on the natural time scale of molecular dynamics. Since on this time scale the probability for spontaneous emission is quite small, it is far from straightforward to determine its leading order expression and to show that the remainder terms are even smaller. In particular we need to carefully separate the three time-scales given by the slow nuclear motion, the intermediate electronic motion and the fast photons. The main idea of our proof is the construction of subspaces that correspond to specific electronic states relative to the nucleonic configuration and momentum that are dressed by a cloud of virtual photons. The restriction of the full dynamics to these subspaces is the Born-Oppenheimer approximation. A transition between two subspaces corresponds to a change in the electronic state with simultaneous emission or absorption of a free photon.

Before we can make these ideas more precise, we have to explain the mathematical 
model. For a molecule with $l$ nuclei and $r$ electrons the Hamiltonian is (in atomic units where $\hbar=1$ and $c=1$ )

$$
H_{\mathrm{mol}}=-\frac{1}{2 m_{\mathrm{nuc}}} \sum_{j=1}^{l} \Delta_{x_{j}}-\frac{1}{2 m_{\mathrm{el}}} \sum_{j=1}^{r} \Delta_{y_{j}}+\alpha\left[V_{\mathrm{ee}}(y)+V_{\mathrm{en}}(x, y)+V_{\mathrm{nn}}(x)\right]
$$

acting on the state space $\mathcal{H}_{\text {mol }}:=\mathcal{H}_{\text {nuc }} \otimes \mathcal{H}_{\mathrm{el}}:=L^{2}\left(\mathbb{R}_{x}^{3 l}\right) \otimes L_{\text {as }}^{2}\left(\mathbb{R}_{y}^{3 r}\right)$. Here $x=\left(x_{1}, \ldots, x_{l}\right)$ denotes the configuration of the $l$ nuclei, $y=\left(y_{1}, \ldots, y_{r}\right)$ the configuration of the $r$ electrons and $\alpha$ is the fine structure constant

$$
\alpha:=\frac{e^{2}}{4 \pi} \approx \frac{1}{137}
$$

For notational simplicity we assume that all the nuclei have the same mass $m_{\text {nuc }}$ and denote the electron mass by $m_{\mathrm{el}}$. We also disregard spin as it would only complicate notation and not change the results. For the moment let $V_{\text {ee }}, V_{\text {en }}$ and $V_{\text {nn }}$ be the Coulomb potentials between electrons, electrons and nuclei, and nuclei respectively.

Taking into account also the coupling to the quantized radiation field, the Hamiltonian for the system becomes

$$
\begin{aligned}
\tilde{H}:= & \frac{1}{2 m_{\mathrm{nuc}}} \sum_{j=1}^{l}\left(p_{j, x}-2 \pi^{\frac{1}{2}} \alpha^{\frac{1}{2}} Z_{j} A_{\Lambda}\left(x_{j}\right)\right)^{2}+\frac{1}{2 m_{\mathrm{el}}} \sum_{j=1}^{r}\left(p_{j, y}-2 \pi^{\frac{1}{2}} \alpha^{\frac{1}{2}} A_{\Lambda}\left(y_{j}\right)\right)^{2} \\
& +H_{\mathrm{f}}+\alpha\left[V_{\mathrm{ee}}(y)+V_{\mathrm{en}}(x, y)+V_{\mathrm{nn}}(x)\right]
\end{aligned}
$$

where $p_{j, x}:=-\mathrm{i} \nabla_{x_{j}}$ and $p_{j, y}:=-\mathrm{i} \nabla_{y_{j}}$. It acts on the Hilbert space

$$
\mathcal{H}:=\mathcal{H}_{\text {mol }} \otimes \mathscr{F}
$$

where $\mathscr{F}$ is the photonic Fock space.

The nuclear charge in multiples of the electron charge is denoted by $Z_{j}, H_{\mathrm{f}}$ is the Hamiltonian of the free field and $A_{\Lambda}$ is the quantized transverse vector potential in the Coulomb gauge, with a sharp ultraviolet cutoff $\Lambda$, needed to make the Hamiltonian $\tilde{H}$ a well-defined self-adjoint operator. More explicitly

$$
A_{\Lambda}(q)=\frac{1}{(2 \pi)^{\frac{3}{2}}} \sum_{\lambda=1}^{2} \int_{|k| \leq \Lambda} \frac{\mathrm{d}^{3} k}{\sqrt{2|k|}} e_{\lambda}(k)\left(\mathrm{e}^{\mathrm{i} k \cdot q} a(k, \lambda)+\mathrm{e}^{-\mathrm{i} k \cdot q} a^{*}(k, \lambda)\right), q \in \mathbb{R}^{3}
$$

where $a^{*}(k, \lambda)$ and $a(k, \lambda)$ are the standard creation and annihilation operators and $\left\{e_{\lambda}\right\}_{\lambda=1,2}$ are the photon polarization vectors. Note that we use a sharp ultraviolet cutoff just to simplify notation. All our proofs work without any changes for smooth cutoffs. Physically the cutoff is irrelevant for the problem at hand, as long as it is large compared to the energy of the emitted photons.

As explained before, the validity of the Born-Oppenheimer approximation rests on the fact that the coupling to the field is small and we will be interested in the asymptotics for small $\alpha$. However, since also the Coulomb interaction depends on $\alpha$ (it is a 
consequence of coupling to the field after all) the size of an atom or molecule as well as the electronic energy levels depend on $\alpha$. To understand atoms and molecules by perturbation theory in $\alpha$ one thus switches to $\alpha$-dependent units where the typical sizes and the typical energies are independent of $\alpha$ : introducing the Bohr-radius $\eta$ and the Rydberg-energy $\mu$ as

$$
\eta=\frac{1}{2 m_{\mathrm{el}} \alpha}, \quad \mu=2 m_{\mathrm{el}} \alpha^{2},
$$

one implements the change of units on the one-particle configuration space as

$$
U_{\eta}: L^{2}\left(\mathbb{R}_{z}^{3}, \mathrm{~d} z\right) \rightarrow L^{2}\left(\mathbb{R}_{z}^{3}, \mathrm{~d} z\right), \quad\left(U_{\eta} \psi\right)(z)=\eta^{3 / 2} \psi(\eta z)
$$

and on the one-photon momentum space as

$$
U_{\mu}: L^{2}\left(\mathbb{R}_{k}^{3}, \mathrm{~d} k\right) \rightarrow L^{2}\left(\mathbb{R}_{k}^{3}, \mathrm{~d} k\right), \quad\left(U_{\mu} \phi\right)(k)=\mu^{3 / 2} \phi(\mu k) .
$$

These transformations are canonically lifted to a unitary $U_{\alpha}$ on the full Hilbert space $\mathcal{H}$. Finally we fix the ultraviolet cutoff in units of Rydberg to some finite value $\Lambda_{0}<\infty$,

$$
\Lambda=\mu \Lambda_{0}
$$

A straightforward computation shows that in the new units the Hamiltonian becomes

$$
\begin{aligned}
H^{\varepsilon, \alpha}:=\frac{1}{\mu} U_{\alpha} \tilde{H} U_{\alpha}^{*}= & \varepsilon^{2} \sum_{j=1}^{l}\left(p_{j, x}-2 \pi^{1 / 2} \alpha^{3 / 2} Z_{j} A_{\Lambda_{0}}\left(\alpha x_{j}\right)\right)^{2} \\
& +\sum_{j=1}^{r}\left(p_{j, y}-2 \pi^{1 / 2} \alpha^{3 / 2} A_{\Lambda_{0}}\left(\alpha y_{j}\right)\right)^{2} \\
& +H_{\mathrm{f}}+V_{\mathrm{ee}}(y)+V_{\mathrm{en}}(x, y)+V_{\mathrm{nn}}(x),
\end{aligned}
$$

where we abbreviate

$$
\varepsilon:=\left(\frac{m_{\mathrm{el}}}{m_{\mathrm{nuc}}}\right)^{1 / 2} .
$$

Note that even for the lightest nuclei $\varepsilon$ is already rather small,

$$
\varepsilon \approx\left\{\begin{array}{ll}
\frac{1}{43} & m_{\mathrm{nuc}}=m_{\mathrm{p}}, \\
\frac{1}{136} & m_{\mathrm{nuc}}=10 m_{\mathrm{p}},
\end{array} \quad m_{\mathrm{p}}: \text { proton mass } .\right.
$$

The physical Hamiltonian (1) depends on the two small dimensionless parameters $\varepsilon$ and $\alpha$. The smallness of $\varepsilon$ is the basis for the Born-Oppenheimer approximation in molecular dynamics and the smallness of $\alpha$ allows for a perturbative understanding of electronic resonances.

Our aim is to construct a Born-Oppenheimer expansion for the resonances of the molecular system. To explain exactly what we mean by this statement we first recall some known results about the two limit cases which are contained in the Hamiltonian $H^{\varepsilon, \alpha}$, the case $\varepsilon=0, \alpha \neq 0$ (a molecule coupled to electromagnetic field with clamped nuclei) and the case $\varepsilon \neq 0, \alpha=0$ (a molecule with dynamical nuclei but no coupling to the field). 


\subsection{Electronic resonances for fixed nuclei $(\varepsilon=0, \alpha>0)$}

When $\varepsilon=0$, the Hamiltonian depends parametrically on the nuclear configuration $x$ and becomes

$$
H^{\varepsilon=0, \alpha}(x)=: H_{\mathrm{el}}(x)+H_{\mathrm{f}}+W\left(\alpha, \Lambda_{0}\right),
$$

where the electronic Hamiltonian

$$
H_{\mathrm{el}}(x):=\sum_{j=1}^{r} p_{j, y}^{2}+V_{\mathrm{ee}}(y)+V_{\mathrm{en}}(x, y)+V_{\mathrm{nn}}(x)
$$

is for every fixed $x$ a self-adjoint operator on $\mathcal{H}_{\mathrm{el}}=L^{2}\left(\mathbb{R}_{y}^{3 r}\right)$. We assume that the spectrum of $H_{\mathrm{el}}(x)$ is of the form

$$
\sigma\left(H_{\mathrm{el}}(x)\right)=\left\{E_{0}(x), E_{1}(x), \ldots\right\} \cup[\Sigma(x), \infty),
$$

where $E_{0}(x)<E_{1}(x)<E_{2}(x)<\ldots \leq \Sigma(x)$ are eigenvalues of finite multiplicity below $\Sigma(x)$, possibly with an accumulation point at $\Sigma(x)$ and absolutely continuous spectrum in $[\Sigma(x),+\infty)$. As shown in [Zi], if

$$
r \leq \sum_{j=1}^{l} Z_{j}
$$

then $H_{\mathrm{el}}(x)$ has an infinite number of eigenvalues below the threshold $\Sigma(x)$.

Under the same hypothesis (3) it was shown in [LiLo, using a binding condition introduced in [GLL], that $H^{\varepsilon=0, \alpha}$ has a ground state $E(x)$ for every $\alpha>0$ (using a smooth ultraviolet cutoff). The existence of the ground state for small values of the fine structure constant $\alpha$ has been shown before in [BFS].

It is expected that the electronic eigenvalues $E_{1}(x), E_{2}(x), \ldots$ turn into resonances and that apart from the ground state the spectrum of $H^{\varepsilon=0, \alpha}$ is absolutely continuous. It was shown in [BFS] (see also [AFFS, $[\mathrm{HHH}$ ) for the case $l=1$ (an atom) that the eigenvalues $\left\{E_{j}\right\}_{j>0}$ become resonances in the sense of the Aguilar-Balslev-CombesSimon theory ([HiSi] chapters 16-18, $\mathrm{ReSi}_{4}$ sections XII.6, XIII.10, [Si]). We quote a typical result (cf. e.g. Corollary 2 from $[\mathrm{HHH}]$ ) on the exponential decay of resonant states without giving technical details.

Almost exponential decay of atomic resonances. Let $P_{j}$ be the spectral projection of $H_{\mathrm{el}}=H_{\mathrm{el}}(0)$ corresponding to the eigenvalue $E_{j}, j \neq 0$, and let $Q_{0}$ be the projection on the Fock-vacuum. For $\Psi \in \operatorname{Ran} P_{j} \otimes Q_{0}$ normalized to one it holds that

$$
\left\|\left(P_{j} \otimes Q_{0}\right) \mathrm{e}^{-\mathrm{i} t H^{\varepsilon=0, \alpha}} \Psi\right\|^{2}=\mathrm{e}^{-t \alpha^{3} \gamma}+b(\alpha, t),
$$

where $b(\alpha, t)=\mathcal{O}\left(\alpha^{\frac{1}{2}}\right)$ uniformly in time and $\gamma>0$. It follows that the lifetime of the resonance is of order $\alpha^{-3}$.

The difficult part in proving such results is to control the error term uniformly in time. For short times $t \ll \alpha^{-3}$ the decay rate into any other state $E_{i}<E_{j}$ can be easily computed by a perturbative argument known as Fermi's golden rule. 
Fermi's golden rule. Let $P_{j}$ and $P_{i}$ be the spectral projections of $H_{\mathrm{el}}=H_{\mathrm{el}}(0)$ corresponding to the eigenvalues $E_{i}<E_{j}$ and let $Q_{0}$ be the projection on the Fock-vacuum. Then for $\Psi \in \operatorname{Ran} P_{j} \otimes Q_{0}$ normalized to one it holds that

$$
\left\|\left(P_{i} \otimes 1\right) \mathrm{e}^{-\mathrm{i} t H^{\varepsilon=0, \alpha}} \Psi\right\|^{2}=\frac{4}{3} \alpha^{3}\left(E_{j}-E_{i}\right)^{3}\left|D_{i j}\right|^{2} t+o\left(\alpha^{3}\right)
$$

uniformly on bounded time intervals, where $\left|D_{i j}\right|$ is the dipole-matrix element, c.f. Section 1.3 .

Since the natural time scale for nuclear dynamics is short in this sense, we will not be interested in results on exponential decay on long time scales for dynamical molecules (it is not even clear what this would exactly mean), but in explicit decay rates in the form of Fermi's golden rule. However, as will be explained in Section 1.3, for moving nuclei the decay rate depends on the configuration of the nuclei, which in turn changes quickly on the time scale of the resonance. As a consequence one can not just adapt the usual perturbative argument in order to compute decay rates for dynamical molecules. But before coming to the full problem, let us first recall some basic facts about the case $\alpha=0$ and $\varepsilon>0$.

\subsection{Dynamical nuclei without coupling to the field}

$$
(\varepsilon>0, \alpha=0)
$$

This case is the setting of the standard time-dependent Born-Oppenheimer approximation (e.g. $\left.\mathrm{HaJ}_{1}, \mathrm{MaSo}_{1}, \mathrm{SpTe}, \mathrm{Teu}_{2}, \mathrm{PST}_{1}, \mathrm{MaSo}_{2}\right]$ ). The Hamiltonian has the form

$$
H^{\varepsilon, \alpha=0}=-\varepsilon^{2} \Delta_{x}+H_{\mathrm{el}}(x),
$$

where we omit the field Hamiltonian $H_{\mathrm{f}}$ because it commutes with the rest and is therefore irrelevant.

For kinetic energies of order one (in units of Rydberg!) the nuclei have velocities of order $\varepsilon$. The time scale on which the nuclei move distances of order one (in units of Bohr radii) are thus times of order $\varepsilon^{-1}$. Hence it is natural to change the unit of time as well and to solve the time-dependent Schrödinger equation

$$
\mathrm{i} \varepsilon \frac{\mathrm{d}}{\mathrm{d} t} \psi(t)=H^{\varepsilon, \alpha=0} \psi(t) .
$$

The long time-scale will be reflected in the following by the fact, that we evaluate unitary groups at times $\frac{t}{\varepsilon}$, i.e. we consider $\mathrm{e}^{-\mathrm{i} H \frac{t}{\varepsilon}}$ for $t$ of order one.

To avoid additional technicalities, one assumes that $H_{\mathrm{el}}$ is in a suitable sense a smooth function of $x$. This requires to introduce a smearing of the nuclear charge distribution $\varphi \in C_{0}^{\infty}\left(\mathbb{R}^{3}\right)$ with $\varphi \geq 0$ and $\int \varphi=1$. The electronic repulsion remains unchanged

$$
V_{\mathrm{ee}}(y)=\sum_{n=1}^{r-1} \sum_{m=n+1}^{r} \frac{1}{\left|y_{n}-y_{m}\right|}
$$

while the electron-nucleon attraction and the nuclear repulsion become

$$
V_{\text {en }}(x, y)=-\sum_{n=1}^{r} \sum_{m=1}^{l}\left(\frac{2}{\pi}\right)^{1 / 2} Z_{m} \int_{\mathbb{R}^{3}} \mathrm{~d} k \frac{\hat{\varphi}(k)}{|k|^{2}} \mathrm{e}^{\mathrm{i} k \cdot\left(y_{n}-x_{m}\right)}
$$


and

$$
V_{\mathrm{nn}}(x)=\sum_{n=1}^{l-1} \sum_{m=n+1}^{l} 4 \pi Z_{n} Z_{m} \int_{\mathbb{R}^{3}} \mathrm{~d} k \frac{|\hat{\varphi}(k)|^{2}}{|k|^{2}} \mathrm{e}^{\mathrm{i} k \cdot\left(x_{n}-x_{m}\right)} .
$$

In $\mathrm{MaSo}_{2}$ a "twisted" pseudo-differential calculus is introduced, which, generalizing Hunziker's distortion analyticity method [Hu], allows to treat also the case of the unsmeared Coulomb potential.

Let $E_{j}(x)$ be an eigenvalue of the electronic Hamiltonian $H_{\mathrm{el}}(x)$ which is globally isolated by a gap from the rest of the spectrum.

Definition of isolated electronic eigenvalues. Let for all $x \in \mathbb{R}^{3 l}$ be $E_{j}(x)$ an eigenvalue of the electronic Hamiltonian $H_{\mathrm{el}}(x)$. The family $E_{j}(x)$ is called isolated, if there exist two functions $f_{ \pm} \in C_{\mathrm{b}}\left(\mathbb{R}_{x}^{3 l}, \mathbb{R}\right)$ defining an interval $I(x)=\left[f_{-}(x), f_{+}(x)\right]$ such that

$$
\sigma\left(H_{\mathrm{el}}(x)\right) \cap I(x)=E_{j}(x) \quad \text { and } \quad \inf _{x \in \mathbb{R}^{3 l}} \operatorname{dist}\left(E_{j}(x), \sigma\left(H_{\mathrm{el}}(x)\right) \backslash E_{j}(x)\right)>0 .
$$

This condition implies that $E_{j}(x)$ and the spectral projection $P_{j}(x)$ onto the eigenspace of $E_{j}(x)$ are smooth functions of $x$, c.f. Lemma 4. We denote by $P_{j}$ the direct integral

$$
P_{j}:=\int_{\mathbb{R}^{3 l}}^{\oplus} \mathrm{d} x P_{j}(x)
$$

which acts on $\mathcal{H}_{\text {nuc }} \otimes \mathcal{H}_{\mathrm{el}} \cong L^{2}\left(\mathbb{R}^{3 l}, \mathcal{H}_{\mathrm{el}}\right)$.

The Born-Oppenheimer approximation rests on the observation that the electronic state adjusts adiabatically to the slow motion of the nuclei, i.e., that the subspace $P_{j} \mathcal{H}_{\text {mol }}$ is approxiamtely invariant under the time evolution.

A rigorous version of this statement is the following theorem from [SpTe, which is also a special case of Proposition 1 proven below.

Leading order Born-Oppenheimer approximation. The operator

$$
H_{j}^{\varepsilon}:=P_{j} H^{\varepsilon, \alpha=0} P_{j}+\left(1-P_{j}\right) H^{\varepsilon, \alpha=0}\left(1-P_{j}\right)
$$

is self-adjoint on the domain $D$ of $H^{\varepsilon, \alpha=0}$ and satisfies

$$
\left\|\mathrm{e}^{-\mathrm{i} \frac{t}{\varepsilon} H^{\varepsilon, \alpha=0}}-\mathrm{e}^{-\mathrm{i} \frac{t}{\varepsilon} H_{j}^{\varepsilon}}\right\|_{\mathcal{L}\left(D, \mathcal{H}_{\mathrm{mol}}\right)}=\mathcal{O}(\varepsilon|t|+\varepsilon) .
$$

This result is optimal in the sense that the difference is not smaller than order $\varepsilon$. However, the overlap

$$
\left\|P_{i} \mathrm{e}^{-\mathrm{i} \frac{t}{\varepsilon} H^{\varepsilon, \alpha=0}} P_{j}\right\|=\mathcal{O}(\varepsilon) \quad \text { for } i \neq j
$$

that the true time evolution introduces between the different electronic subspaces does not correspond to actual transitions between electronic states. Indeed, the subspaces $P_{j} \mathcal{H}_{\text {mol }}$ can be replaced by slightly deformed superadiabatic subspaces $P_{j}^{\varepsilon} \mathcal{H}_{\text {mol }}$ that are invariant to higher order in $\varepsilon$. Physically in $P_{j}^{\varepsilon} \mathcal{H}_{\mathrm{mol}}$ the electronic state now depends also on the velocity of the nuclei. 
We construct such superadiabatic projections in Proposition 2, A straightforward consequence is the following statement.

Second order Born-Oppenheimer approximation. The operator

$$
\tilde{H}_{j}^{\varepsilon}:=P_{j}^{\varepsilon} H^{\varepsilon, \alpha=0} P_{j}^{\varepsilon}+\left(1-P_{j}^{\varepsilon}\right) H^{\varepsilon, \alpha=0}\left(1-P_{j}^{\varepsilon}\right)
$$

is self-adjoint on the domain $D$ of $H^{\varepsilon, \alpha=0}$ and satisfies

$$
\left\|\left(\mathrm{e}^{-\mathrm{i} \frac{t}{\varepsilon} H^{\varepsilon, \alpha=0}}-\mathrm{e}^{-\mathrm{i} \frac{\mathrm{t}}{\varepsilon} \tilde{H}_{j}^{\varepsilon}}\right) \mathbf{1}_{(-\infty, E)}\left(H^{\varepsilon, \alpha=0}\right)\right\|_{\mathcal{L}\left(\mathcal{H}_{\mathrm{mol}}\right)}=\mathcal{O}\left(\varepsilon^{2}|t|\right) .
$$

Here an energy cutoff at an arbitrary but fixed energy $E$ is needed. This improved approximation of the dynamics is necessary for obtaining error terms smaller than the effect we are interested in, namely transitions between different electronic levels due to spontaneous emission of photons. But it turns out that a rigorous control of these error terms requires to prove (5) and (6) with respect to more general energy norms, which is the main new content of Propositions 1 and 2.

On the other hand, (6) can be shown with an error of order $\varepsilon^{N}$ for any $N \in \mathbb{N}$. Martinez and Sordoni even prove exponential error bounds without assuming a regularization on the nuclear charges, $\mathrm{MaSO}_{2}$. However, the task of computing the exponentially small transition probabilities between superadiabatic subspaces (transitions that happen without emission of photons) is extremely difficult even on a heuristic level, see $\mathrm{HaJ}_{2}, \mathrm{BGT}, \mathrm{BeGo}$.

Another question is, whether one can dispose with the gap condition. At crossings of electronic eigenvalues the Born-Oppenheimer approximation breaks down and transitions between the levels occur at order $\varepsilon^{0}$, cf. [LaTe] and references therein. For eigenvalues embedded into or at the threshold to continuous spectrum the rate of transition depends on the details of the model (see e.g. [Ten] and [TeTe]).

Finally we remark that the importance of the Born-Oppenheimer approximation lies in the observation that the diagonal Hamiltonian $H_{j}^{\varepsilon}$, when acting on states in the range of $P_{j}$, has an asymptotic expansion starting with very simple terms,

$$
H_{j}^{\varepsilon} P_{j}=\left(-\left(\varepsilon \nabla_{x}^{\text {Berry }}\right)^{2}+E_{j}(x)\right) P_{j}+\mathcal{O}\left(\varepsilon^{2}\right) .
$$

Here $\nabla_{x}^{\text {Berry }}:=P_{j} \nabla_{x} P_{j}$ is the so-called Berry connection. Note that the electronic eigenvalue $E_{j}(x)$ appears as an effective potential for the motion of the nuclei. To get the correct higher order terms, one needs to expand $\tilde{H}_{j}^{\varepsilon}$ on the range of $P_{j}^{\varepsilon}$ instead. While at zeroth and first order one obtains the same expansion as for $H_{j}^{\varepsilon} P_{j}$, starting at second order additional terms appear, see for example in $\left[\mathrm{PST}_{2}\right.$. Thus the unitary groups $\mathrm{e}^{-\mathrm{i} \frac{t}{\varepsilon} H_{j}^{\varepsilon}} P_{j}$ resp. $\mathrm{e}^{-\mathrm{i} \frac{t}{\varepsilon} \tilde{H}_{j}^{\varepsilon}} P_{j}^{\varepsilon}$ can be computed by solving a Schrödinger equation for the nuclei only. Note that (5) and (6) are indeed the nontrivial mathematical statements to prove for justifying the time-dependent Born-Oppenheimer approximation. 


\subsection{Dynamical nuclei with coupling to the field $(\varepsilon, \alpha>0)$}

The coupling to the quantized radiation field presumably turns all electronic eigenvalues except for the ground state into resonances. Our first aim is to prove that the BornOppenheimer approximation for a molecule described by $H^{\varepsilon, \alpha}$ remains valid. This makes sense only if the lifetime of the resonance, given according to the above discussion by $\alpha^{-3}$, is bigger than the time scale of molecular dynamics, given by $\varepsilon^{-1}$. To control the relation between the two scales we thus choose $\alpha$ to be a function of $\varepsilon$ such that $\alpha(\varepsilon)^{-3}>\varepsilon^{-1}$. Assuming that

$$
\alpha(\varepsilon)=\varepsilon^{\beta}, \quad \beta>0,
$$

this condition implies that $\beta>\frac{1}{3}$. This is always true for realistic nuclei because

$$
m_{\mathrm{p}} \leq m_{\mathrm{nuc}} \leq 250 m_{\mathrm{p}} \quad \text { corresponds to } \quad \varepsilon_{\min }=\frac{1}{680} \leq \varepsilon \leq \frac{1}{43}=\varepsilon_{\max },
$$

where $m_{\mathrm{p}}$ is the proton mass. Thus

$$
\beta_{\text {min }}=\frac{\ln \alpha}{\ln \varepsilon_{\text {min }}} \approx 0.75 \text { and } \beta_{\max }=\frac{\ln \alpha}{\ln \varepsilon_{\max }} \approx 1.31,
$$

which suggests to consider $\frac{3}{4}<\beta<\frac{4}{3}$. For some results we are able to cover even the range $\frac{2}{3}<\beta<\frac{4}{3}$, while for others we have to restrict to $\frac{5}{6}<\beta<\frac{4}{3}$ which corresponds to $m_{\mathrm{p}} \leq m_{\mathrm{nuc}} \leq 72 m_{\mathrm{p}}$.

Inserting (7) into $H^{\varepsilon, \alpha}$ as given in (1) and expanding in powers of $\varepsilon$, we get a Hamiltonian which depends just on $\varepsilon$. Setting

$$
\begin{aligned}
H_{0}^{\varepsilon}:= & \varepsilon^{2} \sum_{j=1}^{l} p_{j, x}^{2}+H_{\mathrm{el}}(x)+H_{\mathrm{f}} \\
H_{1}^{\varepsilon}:= & -4 \pi^{1 / 2} \sum_{j=1}^{r} A\left(\varepsilon^{\beta} y_{j}\right) \cdot p_{j, y} \\
H_{2}^{\varepsilon}:= & -4 \pi^{1 / 2} \sum_{j=1}^{l} Z_{j} A\left(\varepsilon^{\beta} x_{j}\right) \cdot \varepsilon p_{j, x} \\
& +\varepsilon^{\frac{3}{2} \beta-1} 4 \pi \sum_{j=1}^{r}: A\left(\varepsilon^{\beta} y_{j}\right)^{2}:+\varepsilon^{\frac{3}{2} \beta+1} 4 \pi \sum_{j=1}^{l} Z_{j}^{2}: A\left(\varepsilon^{\beta} x_{j}\right)^{2}:,
\end{aligned}
$$

where we normal ordered the quadratic terms, we can write $H^{\varepsilon}:=H^{\varepsilon, \alpha(\varepsilon)}$ as

$$
H^{\varepsilon}=H_{0}^{\varepsilon}+\varepsilon^{\frac{3}{2} \beta} H_{1}^{\varepsilon}+\varepsilon^{\frac{3}{2} \beta+1} H_{2}^{\varepsilon} .
$$

Note that we think of $\varepsilon p_{j, x}$ being of order $\varepsilon^{0}$, since we want to look at states with nuclear kinetic energy of order $\varepsilon^{0}$. The leading order term $H_{0}^{\varepsilon}$ contains no coupling to the field 
at all. The first order term $H_{1}^{\varepsilon}$ describes the linear coupling of the electrons to the field and will be the relevant term for understanding spontaneous emission of photons. Contributions from $H_{2}^{\varepsilon}$ will always be of lower order and contribute only to our error terms.

Lemma 1 below asserts that $H^{\varepsilon}$ is a well-defined self-adjoint operator for $\varepsilon$ sufficiently small and that the expansion (8) makes actually sense, since the coefficients $H_{1}^{\varepsilon}$ and $H_{2}^{\varepsilon}$ are relatively $H_{0}^{\varepsilon}$-bounded with relative bounds independent of $\varepsilon$.

We come now to an informal statement of our main results. Let

$$
H_{j, \text { field }}^{\varepsilon}:=H_{j}^{\varepsilon} \otimes 1+1 \otimes H_{\mathrm{f}} .
$$

In Corollary 1 we show that, up to a worse error estimate, the statement of (5) remains valid.

\section{Leading order BO-approximation with coupling to the field.}

For $\frac{2}{3}<\beta \leq \frac{4}{3}$ it holds that

$$
\left\|\mathrm{e}^{-\mathrm{i} \frac{t}{\varepsilon} H^{\varepsilon}}-\mathrm{e}^{-\mathrm{i} \frac{t}{\varepsilon} H_{j, \text { field }}^{\varepsilon}}\right\|_{\mathcal{L}\left(D_{0}, \mathcal{H}\right)}=\mathcal{O}\left(\varepsilon^{\frac{3}{2} \beta-1}|t|+\varepsilon\right) .
$$

Technically this is a straightforward perturbative consequence of (5), as the contribution of $\varepsilon^{\frac{3}{2} \beta} H_{1}^{\varepsilon}$ is of order $\varepsilon^{\frac{3}{2} \beta-1}$ for times of order $\varepsilon^{-1}$. However, we still believe that this result is conceptually important. It shows that, in the context where the Born-Oppenheimer approximation is usually applied, the coupling to the radiation field is negligible at leading order. To our knowledge this is the first mathematical result of this type.

Our main result concerns the failure of the Born-Oppenheimer approximation because of spontaneous emission of photons. However, as we will show, the probability for making a transition through spontaneous emission is of order $\varepsilon^{3 \beta-1}|t|$, which for $\beta \geq 1$ is smaller than $\varepsilon^{2}$, the square of the error in the standard Born-Oppenheimer approximation (5). Hence for $\beta \geq 1$ we need to consider transitions between the superadiabatic subspaces $P_{j}^{\varepsilon}$ in order to correctly separate transitions through spontaneous emission from errors in the adiabatic approximation. Our main result is then the following, cf. Theorem 2 .

Probability for spontaneous emission. Let $E_{j}(x)>E_{i}(x)$ for all $x$ and let $\Psi=$ $\psi \otimes \Omega$ with $\psi \in \operatorname{Ran} P_{j}^{\varepsilon}$ and $\Omega \in \mathscr{F}$ the vacuum state. The probability for ending up in the $i$-th electronic state after time $t$ when starting in $\Psi$ is

$$
\left\|\left(P_{i}^{\varepsilon} \otimes 1\right) \mathrm{e}^{-\mathrm{i} \frac{t}{\varepsilon} H^{\varepsilon}} \Psi\right\|^{2}=\varepsilon^{3 \beta-1} \int_{0}^{t} \frac{4}{3}\left\|\left|D_{i j}\right| \Delta_{E}^{3 / 2} \mathrm{e}^{-\mathrm{i} \frac{s}{\varepsilon} H_{j}^{\varepsilon}} P_{j} \psi\right\|^{2} \mathrm{~d} s+o\left(\varepsilon^{3 \beta-1}\right) .
$$

Here $\Delta_{E}$ and $D_{i j}$ are real-valued multiplication operators, namely $\Delta_{E}(x)=E_{j}(x)-E_{i}(x)$ the difference in energy and $D_{i j}(x):=\sum_{k=1}^{r}\left\langle\varphi_{i}(x) \mid y_{k} \varphi_{j}(x)\right\rangle_{\mathcal{H}_{\mathrm{el}}}$ the dipole coupling element. $\varphi_{i}(x)$ and $\varphi_{j}(x)$ are normalized electronic states in $\operatorname{Ran} P_{i}(x)$ and $\operatorname{Ran} P_{j}(x)$ respectively.

Thus the decay probability can be computed by propagating the initial molecular 
wave function according to the standard Born-Oppenheimer approximation in the level $E_{j}$ and integrating the decay rate along this trajectory. The decay rate is given by $\frac{4}{3} \alpha^{3} \varepsilon^{-1}\left|D_{i j}(x)\right|^{2} \Delta_{E}(x)^{3}$ as a function of the nuclear configuration $x$. Recall that $\alpha=\varepsilon^{\beta}$ and that we rescaled time by $\varepsilon^{-1}$. This is the natural generalization of Fermi's golden rule for atoms, c.f. (4), to moving nuclei.

We briefly sketch the strategy of our proof and comment on some difficulties. The basic idea is to use time-dependent perturbation theory according to the splitting

$$
H^{\varepsilon}=H_{j \text {,ield }}^{\varepsilon}+\varepsilon^{\frac{3}{2} \beta} H_{1}^{\varepsilon}+\mathcal{O}\left(\varepsilon^{\frac{3}{2} \beta+1}\right) .
$$

Note that the $\mathcal{O}\left(\varepsilon^{2}\right)$ term contains the $H_{2}^{\varepsilon}$ term, i.e. higher order terms in the coupling to the field, and error terms from the Born-Oppenheimer approximation, i.e. $P_{j}^{\varepsilon} H^{\varepsilon}\left(1-P_{j}^{\varepsilon}\right)$ and its adjoint. Abbreviating $P_{j, 0}^{\varepsilon}:=P_{j}^{\varepsilon} \otimes Q_{0}$, first order time-dependent perturbation theory gives at least formally

$$
\begin{aligned}
& P_{i}^{\varepsilon} \mathrm{e}^{-\mathrm{i} \frac{t}{\varepsilon} H^{\varepsilon}} P_{j, 0}^{\varepsilon}=\underbrace{P_{i}^{\varepsilon} \mathrm{e}^{-\mathrm{i} \frac{t}{\varepsilon} H_{j, \text { field }}^{\varepsilon}} P_{j, 0}^{\varepsilon}}_{=0}-\frac{\mathrm{i} \varepsilon^{\frac{3}{2} \beta}}{\varepsilon} P_{i}^{\varepsilon} \int_{0}^{t} \mathrm{e}^{-\mathrm{i} \frac{t-s}{\varepsilon} H_{j, \text { field }}^{\varepsilon}} H_{1}^{\varepsilon} \mathrm{e}^{-\mathrm{i} \frac{s}{\varepsilon} H_{j, \text { field }}^{\varepsilon}} P_{j, 0}^{\varepsilon} \mathrm{d} s+\mathcal{O}\left(\varepsilon^{\frac{3}{2} \beta}\right) \\
& =-\mathrm{i} \varepsilon^{\frac{3}{2} \beta-1} \underbrace{\int_{0}^{t} \mathrm{e}^{-\mathrm{i} \frac{t-s}{\varepsilon} H_{i, \text { field }}} P_{i}^{\varepsilon} H_{1}^{\varepsilon} P_{j, 0}^{\varepsilon} \mathrm{e}^{-\mathrm{i} \frac{s}{\varepsilon} H_{j, \text { field }}^{\varepsilon}} \mathrm{d} s}_{(*)}+\mathcal{O}\left(\varepsilon^{\frac{3}{2} \beta}\right) .
\end{aligned}
$$

This integral expression is certainly a correct formula for the leading order piece of the wave function that made a transition after time $t$. However, since $P_{i}^{\varepsilon} H_{1}^{\varepsilon} P_{j, 0}^{\varepsilon}$ is of order one, it seems at first sight to be of order $\varepsilon^{\frac{3}{2} \beta-1}$, giving a transition probability of order $\varepsilon^{3 \beta-2}$. This is by a factor of $\varepsilon^{-1}$ larger than the expected value of order $\alpha^{3} \varepsilon^{-1}=\varepsilon^{3 \beta-1}$. Thus the integral $(*)$ must be of order $\varepsilon^{\frac{1}{2}}$ due to oscillations. We don't see any way, however, to evaluate $(*)$ directly in order to get the simple formula $(9)$. This is because the "unperturbed dynamics" given by the Born-Oppenheimer approximation is still a highly nontrivial Schrödinger evolution for many interacting particles. In order to obtain a perturbative integral expression for the leading order transitions that has less oscillations and is thus tractable, we replace $P_{j, 0}^{\varepsilon}$ by dressed superadiabatic vacuum projections $P_{j \text {,vac }}^{\varepsilon}$. Physically speaking, this is because the leading order effect of the coupling to the field is a dressing of the electrons of order $\varepsilon^{\frac{3}{2} \beta}=\alpha^{\frac{3}{2}}$. The rate of spontaneous emission is only of order $\varepsilon^{\frac{3}{2} \beta+\frac{1}{2}}$. However, since the dressing does not grow as a function of time, after times of order $\varepsilon^{-1}$ the spontaneous emission of order $\varepsilon^{\frac{3}{2} \beta-\frac{1}{2}}$ dominates the effect of the dressing. Therefore we can neglect the dressing in the final statement and it appears only in the proof.

\section{The main results}

In this section we only give the main theorems and explain their proofs. The more technical proofs of the propositions and the lemmas are provided in Sections $3 \& 4$ 
respectively. In the first subsection we state a result about the time-dependent BornOppenheimer approximation and show that it remains valid, when we switch on the coupling to the field. In the next subsection we verify that also the superadiabatic subspaces survive in the coupled case. Our central results will be presented in the last two subsections. There we consider the transitions between different energy levels, when there are no free photons in the beginning. In Section 2.3 we derive an expression for the leading order of the transition operator and in Section 2.4 we provide a more explicit formula for the transition rate.

\subsection{Born-Oppenheimer approximation with field}

First we consider only the molecular Hamiltonian

$$
H_{\mathrm{mol}}^{\varepsilon}:=-\varepsilon^{2} \sum_{j=1}^{l} \Delta_{x_{j}}+H_{\mathrm{el}}(x) \quad \text { on } \quad \mathcal{H}_{\mathrm{mol}}:=L^{2}\left(\mathbb{R}_{x}^{3 l}\right) \otimes L_{\mathrm{as}}^{2}\left(\mathbb{R}_{y}^{3 r}\right)
$$

with the usual domain $D_{\text {mol }}:=W^{2,2}\left(\mathbb{R}^{3 l+3 r}\right) \cap \mathcal{H}_{\text {mol }}$. We denote the infimum of the spectrum of $H_{\text {mol }}^{\varepsilon}$ by $e$ and set $\Delta_{x}:=\sum_{j=1}^{l} \Delta_{x_{j}}$. Furthermore, we define $D_{\text {mol }}^{0}:=\mathcal{H}_{\text {mol }}$ and denote the maximal domain of $\left(H_{\mathrm{mol}}^{\varepsilon}\right)^{n}$ equipped with the graph norm by $D_{\mathrm{mol}}^{n}$ for $n \in \mathbb{N}$.

Let $E_{j}(x)$ be an isolated energy band with $H_{\mathrm{el}}(x) P_{j}(x)=E_{j}(x) P_{j}(x)$. We will make use of the following version of the leading order time-dependent Born-Oppenheimer approximation.

Proposition 1. Let $E_{j}$ be an isolated energy band and $P_{j}$ the corresponding band projection. The operator

$$
H_{j}^{\varepsilon}:=P_{j} H_{\mathrm{mol}}^{\varepsilon} P_{j}+\left(1-P_{j}\right) H_{\mathrm{mol}}^{\varepsilon}\left(1-P_{j}\right)
$$

with domain $D_{\mathrm{mol}}$ is self-adjoint and it holds for $n=0,1$ that

$$
\left\|\mathrm{e}^{-\mathrm{i} \frac{t}{\varepsilon} H_{\mathrm{mol}}^{\varepsilon}}-\mathrm{e}^{-\mathrm{i} \frac{t}{\varepsilon} H_{j}^{\varepsilon}}\right\|_{\mathcal{L}\left(D_{\mathrm{mol}}^{n+1}, D_{\mathrm{mol}}^{n}\right)}=\mathcal{O}(\varepsilon|t|+\varepsilon) .
$$

Moreover, $P_{j} \in \mathcal{L}\left(D_{\mathrm{mol}}^{n}\right)$ with norm bounded independently of $\varepsilon$ for all $n \in \mathbb{N}$.

This is a variant of a result in $[\mathrm{SpTe}$. However, in (10) we have a slower growth of the bound as a function of time and better control on the domains, which is essential for the following. The proof given in Section 3.1 is a streamlined and improved version of the approach in SpTe.

Now we will take the radiation field into account. Recall that

$$
H_{0}^{\varepsilon}:=H_{\mathrm{mol}}^{\varepsilon} \otimes 1+1 \otimes H_{\mathrm{f}}
$$

on $D_{0}=\left(D_{\text {mol }} \otimes \mathscr{F}\right) \cap\left(\mathcal{H}_{\text {mol }} \otimes D\left(H_{\mathrm{f}}\right)\right)$ and that

$$
H^{\varepsilon}=H_{0}^{\varepsilon}+\varepsilon^{\frac{3}{2} \beta} H_{1}^{\varepsilon}+\varepsilon^{\frac{3}{2} \beta+1} H_{2}^{\varepsilon}
$$

was defined in (8). The following lemma shows, in particular, that $H_{0}^{\varepsilon}$ and $H^{\varepsilon}$ are self-adjoint on $D_{0}$. 
Lemma 1. The free Hamiltonian

$$
H_{\text {free }}^{\varepsilon}:=\varepsilon^{2} \sum_{j=1}^{l} p_{j, x}^{2}+\sum_{j=1}^{r} p_{j, y}^{2}+H_{\mathrm{f}}
$$

with domain

$$
D_{0}:=D\left(H_{\text {free }}^{\varepsilon}\right)=\left(W^{2,2}\left(\mathbb{R}^{3 l+3 r}\right) \otimes \mathscr{F}\right) \cap\left(\mathcal{H}_{\text {mol }} \otimes D\left(H_{\mathrm{f}}\right)\right)
$$

is self-adjoint. The potentials $V_{\mathrm{ee}}, V_{\mathrm{en}}$ and $V_{\mathrm{nn}}$ are infinitesimally $H_{\text {free }}^{\varepsilon}$ bounded and $H_{1}^{\varepsilon}$

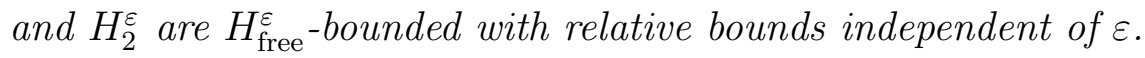

Hence, by Kato-Rellich, $H_{0}^{\varepsilon}$ and $H^{\varepsilon}$ are self-adjoint on the domain $D_{0}$ for $\varepsilon$ small enough.

As before we define the "diagonal" part of $H_{0}^{\varepsilon}$ as

$$
\begin{aligned}
H_{j, \text { field }}^{\varepsilon} & :=\left(P_{j} \otimes 1\right) H_{0}^{\varepsilon}\left(P_{j} \otimes 1\right)+\left(\left(1-P_{j}\right) \otimes 1\right) H_{0}^{\varepsilon}\left(\left(1-P_{j}\right) \otimes 1\right) \\
& =H_{j}^{\varepsilon} \otimes 1+1 \otimes H_{\mathrm{f}} .
\end{aligned}
$$

It is now straightforward to prove the correctness of the leading order Born-Oppenheimer approximation also with field, by treating the coupling to the field as a small perturbation.

Corollary 1. For $n=0,1$ and $\frac{2}{3}<\beta \leq \frac{4}{3}$ it holds that

$$
\left\|\mathrm{e}^{-\mathrm{i} \frac{t}{\varepsilon} H^{\varepsilon}}-\mathrm{e}^{-\mathrm{i} \frac{t}{\varepsilon} H_{j, \text { field }}^{\varepsilon}}\right\|_{\mathcal{L}\left(D_{0}^{n+1}, D_{0}^{n}\right)}=\mathcal{O}\left(\varepsilon^{\frac{3}{2} \beta-1}|t|+\varepsilon\right)
$$

and

$$
\left\|\left(\mathrm{e}^{-\mathrm{i} \frac{t}{\varepsilon} H^{\varepsilon}}-\mathrm{e}^{-\mathrm{i} \frac{t}{\varepsilon} H_{j, \text { field }}}\right)\left(P_{j} \otimes 1\right)\right\|_{\mathcal{L}\left(D_{0}^{n+1}, D_{0}^{n}\right)}=\mathcal{O}\left(\varepsilon^{\frac{3}{2} \beta-1}|t|+\varepsilon\right) .
$$

Proof. Standard time-dependent perturbation theory yields

$$
\left\|\mathrm{e}^{-\mathrm{i} \frac{t}{\varepsilon} H^{\varepsilon}}-\mathrm{e}^{-\mathrm{i} \frac{t}{\varepsilon} H_{0}^{\varepsilon}}\right\|_{\mathcal{L}\left(D_{0}^{n+1}, D_{0}^{n}\right)}=\mathcal{O}\left(\varepsilon^{\frac{3}{2} \beta-1}|t|\right),
$$

because $H^{\varepsilon}-H_{0}^{\varepsilon}=\varepsilon^{\frac{3}{2} \beta}$ in $\mathcal{L}\left(D_{0}^{n+1}, D_{0}^{n}\right)$. The last statement is not completely obvious for $n=1$, but it follows from Lemma 1 and the fact that the commutator $\varepsilon^{-\frac{3}{2} \beta}\left[H^{\varepsilon}-\right.$ $\left.H_{0}^{\varepsilon}, H_{\text {free }}^{\varepsilon}\right]$ is relatively bounded by $\left(H_{\text {free }}^{\varepsilon}\right)^{2}$ uniformly in $\varepsilon$. Now (11) follows from

$$
\left(\mathrm{e}^{-\mathrm{i} \frac{t}{\varepsilon} H_{0}^{\varepsilon}}-\mathrm{e}^{-\mathrm{i} \frac{t}{\varepsilon} H_{j, \text { field }}}\right)=\underbrace{\left(\mathrm{e}^{-\mathrm{i} \frac{t}{\varepsilon} H_{\text {mol }}^{\varepsilon}}-\mathrm{e}^{-\mathrm{i} \frac{t}{\varepsilon} H_{j}^{\varepsilon}}\right)}_{=\mathcal{O}(\varepsilon|t|+\varepsilon) \text { in } \mathcal{L}\left(D_{\text {mol }}^{n+1}, D_{\text {mol }}^{n}\right)} \otimes \mathrm{e}^{-\mathrm{i} \frac{t}{\varepsilon} H_{\mathrm{f}}},
$$

and the fact that $1 \otimes \mathrm{e}^{-\mathrm{i} \frac{t}{\varepsilon} H_{\mathrm{f}}}$ is uniformly bounded in $\mathcal{L}\left(D_{0}^{n}\right)$ and from using the following technical lemma.

Lemma 2. Let $m, n \in \mathbb{N}_{0}$ with $m \leq n$. There is a constant $C<\infty$ such that if $\|B\|_{\mathcal{L}\left(D_{\text {mol }}^{j+n-m}, D_{\text {mol }}^{j}\right)} \leq \delta$ for some $\delta>0$ and all $j=0, \ldots, m$, then $\|B \otimes 1\|_{\mathcal{L}\left(D_{0}^{n}, D_{0}^{m}\right)} \leq C \delta$.

By Proposition 1 we have $P_{j} \in \mathcal{L}\left(D_{\text {mol }}^{n}\right)$ and thus, again by the previous lemma, $P_{j} \otimes 1 \in \mathcal{L}\left(D_{0}^{n}\right)$. This shows also 12 . 


\section{$2.2 \quad$ Superadiabatic subspaces}

At first sight one might hope that the "non-adiabatic matrix elements"

$$
P_{i} \mathrm{e}^{-\mathrm{i} \frac{t}{\varepsilon} H^{\varepsilon}} P_{j}
$$

from the $j$ th to the $i$ th electronic state are, at least for $E_{i}<E_{j}$, dominated by spontaneous emission of photons. However, this is not the case, as the main contribution to (13) comes from a velocity dependent deformation of the electronic eigenstates. More precisely, the range of $P_{j}(x)$ is spanned by the eigenstate for a static nucleonic configuration. But the slow movement of the nuclei will deform the electronic states at order $\varepsilon$. This deformation is visible in the naive "non-adiabatic matrix element" (13), but does not go along with emission of a photon. The projection $P_{j}^{\varepsilon}=P_{j}+\mathcal{O}(\varepsilon)$ onto the correctly modified electronic states is the so-called superadiabatic projection associated to $P_{j}$. As the following proposition shows, the superadiabatic projection $P_{j}^{\varepsilon}$ commutes with $H_{\text {mol }}^{\varepsilon}$ up to errors of order $\varepsilon^{3}$ uniformly on subspaces of bounded total energy. It is possible to go to even higher orders or exponentially small errors of the form $\mathrm{e}^{-\frac{c}{\varepsilon}}$, but this requires some highly technical pseudo-differential calculus, cf. [MaSo 2$]$, and is not needed for our analysis.

Proposition 2. Fix an arbitrary cutoff energy $E<\infty$. For any isolated energy band $E_{j}(x)$ there is $\varepsilon_{0}>0$ such that for $\varepsilon<\varepsilon_{0}$ there are operators $P_{j}^{\varepsilon}$ with the following properties:

$P_{j}^{\varepsilon}$ is an orthogonal projection and for any $n \in \mathbb{N}_{0}$ there is $\varepsilon_{n}>0$ and $C_{n}<\infty$ such that for $\varepsilon<\varepsilon_{n}$ the operators $P_{j}^{\varepsilon}$ are bounded uniformly in $\mathcal{L}\left(D_{\mathrm{mol}}^{n}\right)$ and

$$
\left\|P_{j}^{\varepsilon}-P_{j}\right\|_{\mathcal{L}\left(D_{\text {mol }}^{n}\right)} \leq C_{n} \varepsilon
$$

With $\mathbf{1}_{E}$ denoting the characteristic function on the interval $(-\infty, E]$ we have furthermore that

$$
\left\|\left[H_{\mathrm{mol}}^{\varepsilon}, P_{j}^{\varepsilon}\right] \mathbf{1}_{E}\left(H_{\mathrm{mol}}^{\varepsilon}\right)\right\|_{\mathcal{L}\left(\mathcal{H}_{\mathrm{mol}}, D_{\mathrm{mol}}^{n}\right)} \leq C_{n} \varepsilon^{3}
$$

and

$$
\left\|\left[H_{\mathrm{mol}}^{\varepsilon}, P_{j}^{\varepsilon}\right]\right\|_{\mathcal{L}\left(D_{\mathrm{mol}}^{n+1}, D_{\mathrm{mol}}^{n}\right)} \leq C_{n} \varepsilon .
$$

Given two isolated bands $E_{i}$ and $E_{j}$ the projections $P_{i}^{\varepsilon}$ and $P_{j}^{\varepsilon}$ satisfy

$$
P_{j}^{\varepsilon} P_{i}^{\varepsilon} \mathbf{1}_{E+\frac{1}{2}}\left(H_{\mathrm{mol}}^{\varepsilon}\right)=\mathcal{O}\left(\varepsilon^{2}\right)
$$

in $\mathcal{L}\left(\mathcal{H}_{\mathrm{mol}}, D_{\text {mol }}^{n}\right)$.

Note that it is possible to go to even higher orders or exponentially small errors of the form $\mathrm{e}^{-\frac{c}{\varepsilon}}$, but this requires some highly technical pseudo-differential calculus, cf. $\mathrm{MaSO}_{2}$, and is not needed for our analysis. On the other hand, the statements (14) and (16) without energy cutoffs and also the fact that the errors are small even in the norm of $D_{\mathrm{mol}}^{n}$ for $n>0$ are essential to our analysis. Since they do not follow in any straightforward way from the known results, we give the proof of Proposition 2 in Section 3.2 . 
The next natural steps would be to improve on the error in Proposition 1 for states with high energies cut off and to determine the asymptotic expansion of $P_{j}^{\varepsilon} H_{\mathrm{mol}}^{\varepsilon} P_{j}^{\varepsilon}$. However, for our purpose it suffices to consider the leading order effective Hamiltonian and we will need Proposition 1 without a cutoff. Higher order results can be found e.g. in $\mathrm{MaSo}_{1}, \mathrm{PST}_{2}$.

Now we investigate the lifted projectors $P_{j}^{\varepsilon} \otimes 1 \in \mathcal{L}(\mathcal{H})$. Modulo some technicalities concerning the different graph norms, the following corollary is a simple consequence of Proposition 2

Corollary 2. Let $P_{j}^{\varepsilon}$ be the operator defined in Proposition 2. Then for any $n \in \mathbb{N}_{0}$

$$
\begin{gathered}
\left\|P_{j}^{\varepsilon} \otimes 1\right\|_{\mathcal{L}\left(D_{0}^{n}\right)}=\mathcal{O}(1), \\
\left\|P_{j}^{\varepsilon} \otimes 1-P_{j} \otimes 1\right\|_{\mathcal{L}\left(D_{0}^{n}\right)}=\mathcal{O}(\varepsilon), \\
\left\|\left[H_{0}^{\varepsilon}, P_{j}^{\varepsilon} \otimes 1\right] \mathbf{1}_{E}\left(H_{0}^{\varepsilon}\right)\right\|_{\mathcal{L}\left(\mathcal{H}, D_{0}^{n}\right)}=\mathcal{O}\left(\varepsilon^{3}\right),
\end{gathered}
$$

and for $n \geq 1$

$$
\left\|\left[H_{0}^{\varepsilon}, P_{j}^{\varepsilon} \otimes 1\right]\right\|_{\mathcal{L}\left(D_{0}^{n}, D_{0}^{n-1}\right)}=\mathcal{O}(\varepsilon) .
$$

Proof. In view of Lemma 2, (18) and (19) follow from the corresponding statements for $P_{j}^{\varepsilon}$, and (21) follows from (16) and

$$
\left[H_{0}^{\varepsilon}, P_{j}^{\varepsilon} \otimes 1\right]=\left[H_{\mathrm{mol}}^{\varepsilon}, P_{j}^{\varepsilon}\right] \otimes 1 .
$$

Since $H_{f}$ is nonnegative and $H_{\mathrm{mol}}^{\varepsilon} \otimes 1$ and $1 \otimes H_{\mathrm{f}}$ commute, we have that

$$
\left(\mathbf{1}_{E}\left(H_{\mathrm{mol}}^{\varepsilon}\right) \otimes 1\right) \mathbf{1}_{E}\left(H_{0}^{\varepsilon}\right)=\mathbf{1}_{E}\left(H_{0}^{\varepsilon}\right)
$$

Now (20) is a direct consequence of (15) and the simple computation

$$
\begin{aligned}
\left\|\left[H_{0}^{\varepsilon}, P_{j}^{\varepsilon} \otimes 1\right] \mathbf{1}_{E}\left(H_{0}^{\varepsilon}\right)\right\|_{\mathcal{L}\left(\mathcal{H}, D_{0}^{n}\right)} & =\left\|\left[H_{\mathrm{mol}}^{\varepsilon} \otimes 1, P_{j}^{\varepsilon} \otimes 1\right] \mathbf{1}_{E}\left(H_{0}^{\varepsilon}\right)\right\|_{\mathcal{L}\left(\mathcal{H}, D_{0}^{n}\right)} \\
& \stackrel{(22)}{=}\left\|\left(\left[H_{\mathrm{mol}}^{\varepsilon}, P_{j}^{\varepsilon}\right] \otimes 1\right)\left(\mathbf{1}_{E}\left(H_{\mathrm{mol}}^{\varepsilon}\right) \otimes 1\right) \mathbf{1}_{E}\left(H_{0}^{\varepsilon}\right)\right\|_{\mathcal{L}\left(\mathcal{H}, D_{0}^{n}\right)} \\
& =\left\|\left(\left[H_{\mathrm{mol}}^{\varepsilon}, P_{j}^{\varepsilon}\right] \mathbf{1}_{E}\left(H_{\mathrm{mol}}^{\varepsilon}\right) \otimes 1\right) \mathbf{1}_{E}\left(H_{0}^{\varepsilon}\right)\right\|_{\mathcal{L}\left(\mathcal{H}, D_{0}^{n}\right)} \\
& \leq\left\|\left[H_{\mathrm{mol}}^{\varepsilon}, P_{j}^{\varepsilon}\right] \mathbf{1}_{E}\left(H_{\mathrm{mol}}^{\varepsilon}\right) \otimes 1\right\|_{\mathcal{L}\left(D_{0}^{n}\right)}\left\|\mathbf{1}_{E}\left(H_{0}^{\varepsilon}\right)\right\|_{\mathcal{L}\left(\mathcal{H}, D_{0}^{n}\right)} .
\end{aligned}
$$

In the following, we just write $P_{j}^{\varepsilon}$ for $P_{j}^{\varepsilon} \otimes 1$ to shorten notation.

\subsection{Spontaneous emission: transition operator}

According to Corollary 1 there are no transitions between different electronic states at leading order even on microscopic times of order $\varepsilon^{-1}$. Naive perturbation theory used in the proof suggests that the leading order transitions through coupling to the field are of order $\varepsilon^{\frac{3}{2} \beta-1}$ on this time scale. However, we will see that the transitions through spontaneous emission are actually smaller, namely of order $\varepsilon^{\frac{3}{2} \beta-\frac{1}{2}}$. Since we aim at a 
leading order expression for these transitions, we need to control the full time evolution up to errors which are smaller than $\varepsilon^{\frac{3}{2} \beta-\frac{1}{2}}$. To this end we construct superadiabatic subspaces corresponding to definite dressed electronic states containing so-called virtual but no free photons. Note that the adiabatic subspaces of Corollary 2 correspond to definite electronic states with arbitrary state of the field.

For the sake of clarity we formulate our results about the dressed projector $P_{j \text {,vac }}^{\varepsilon}$ with an additional small parameter $\delta>0$. Later on we will choose a $\delta$ that is fixed by $\varepsilon$ and $\beta$.

Proposition 3. Fix an arbitrary energy cutoff $E<\infty$ and let $E_{j}$ be an isolated electronic energy band. There is $\varepsilon_{0}>0$ such that for $\varepsilon<\varepsilon_{0}$ and any $\delta \geq \varepsilon^{\frac{1}{2}}$ there are operators $P_{j \text {,vac }}^{\varepsilon}(\delta) \in \mathcal{L}(\mathcal{H}) \cap \mathcal{L}\left(D_{0}\right)$ with the following properties:

$P_{j \text {,vac }}^{\varepsilon}$ are orthogonal projections with

$$
\begin{gathered}
\left\|\left[H^{\varepsilon}, P_{j, \mathrm{vac}}^{\varepsilon}\right]\right\|_{\mathcal{L}\left(D_{0}, \mathcal{H}\right)}=\mathcal{O}(\varepsilon), \\
\left\|\left[H^{\varepsilon}, P_{j, \mathrm{vac}}^{\varepsilon}\right] \mathbf{1}_{E+1}\left(H_{0}^{\varepsilon}\right)\right\|_{\mathcal{L}\left(\mathcal{H}, D_{0}\right)}=\mathcal{O}\left(\varepsilon^{\frac{3}{2} \beta} \delta^{\frac{1}{2}}\right),
\end{gathered}
$$

and, as a consequence,

$$
\left\|\left[\tilde{\chi}\left(H^{\varepsilon}\right), P_{j, \mathrm{vac}}^{\varepsilon}\right]\right\|_{\mathcal{L}\left(\mathcal{H}, D_{0}\right)}=\mathcal{O}\left(\varepsilon^{\frac{3}{2} \beta} \delta^{\frac{1}{2}}\right),
$$

for any smooth $\tilde{\chi}$ with compact support in $(-\infty, E+1)$.

Moreover, for $n=0,1$

$$
\left\|P_{j, \text { vac }}^{\varepsilon}-P_{j}^{\varepsilon} \otimes Q_{0}\right\|_{\mathcal{L}\left(D_{0}^{n}\right)}=\mathcal{O}\left(\varepsilon^{\frac{3}{2} \beta} \delta^{-\frac{1}{2}}\right),
$$

where $P_{j}^{\varepsilon}$ is the superadiabatic projection from Proposition 2 and $Q_{0}$ is the vacuum projection in Fock space.

The importance of the dressed vacuum projection $P_{j \text {,vac }}^{\varepsilon}$ lies in the fact that its range is invariant under the full dynamics with a smaller error than $P_{j}^{\varepsilon} \otimes Q_{0}$. This is because it contains also the dressing of the electrons by virtual photons, which is crucial for computing the leading order transitions through spontaneous emission. The transitions from and into the subspace $P_{j \text {,vac }}^{\varepsilon}$ are generated by the commutator $\left[P_{j \text {,vac }}^{\varepsilon}, H^{\varepsilon}\right]$, which we can compute at leading order.

Proposition 4. For $\varepsilon^{\frac{1}{2}} \leq \delta \leq 1$ and $\frac{2}{3}<\beta \leq \frac{4}{3}$

$$
\left[P_{j, \mathrm{vac}}^{\varepsilon}, H^{\varepsilon}\right] \mathbf{1}_{E+\frac{1}{2}}\left(H_{0}^{\varepsilon}\right)=\varepsilon^{\frac{3}{2} \beta} \delta^{\frac{1}{2}} \mathcal{T}_{j} \mathbf{1}_{E+\frac{1}{2}}\left(H_{0}^{\varepsilon}\right)+\mathcal{O}\left(\varepsilon^{\frac{3}{2} \beta+1}\left(1+\varepsilon \delta^{-5 / 2}\right)\right)
$$

in $\mathcal{L}(\mathcal{H})$ with

$$
\mathcal{T}_{j}=\mathrm{i} \delta^{\frac{1}{2}}\left(T_{j}+T_{j}^{*}\right)+2 \delta^{-\frac{1}{2}} \varepsilon\left(\nabla T_{j}+\nabla T_{j}^{*}\right) \cdot \varepsilon \nabla_{x}
$$

and

$$
T_{j}=-\left(H_{\mathrm{f}}+H_{\mathrm{el}}-E_{j}+\mathrm{i} \delta\right)^{-1} H_{1}^{\varepsilon}\left(P_{j} \otimes Q_{0}\right) .
$$

For $n=0,1$ it holds

$$
\left\|\mathcal{T}_{j}\right\|_{\mathcal{L}\left(D_{0}^{n+1}, D_{0}^{n}\right)}=\mathcal{O}(1)
$$


We can now compute the leading order expression for the piece of the evolution that makes a transition from $P_{j}^{\varepsilon} \otimes Q_{0}$ to $P_{i}^{\varepsilon}$, which is the first of our two main results. For the sake of brevity we consider two non-degenerate levels $E_{i}(x)$ and $E_{j}(x)$ with associated normalized eigenfunctions $\varphi_{i}(x) \in \operatorname{Ran} P_{i}(x)$ and $\varphi_{j}(x) \in \operatorname{Ran} P_{j}(x)$. The results can be generalized to degenerate bands in a straightforward manner.

Theorem 1 (Leading order spontaneous emission). Fix an arbitrary energy cutoff $E<\infty$ and let $E_{j}$ and $E_{i}$ be isolated electronic energy bands with $E_{j}-E_{i}>0$ and let $\frac{5}{6}<\beta \leq \frac{4}{3}$. Then

$$
\lim _{\varepsilon \rightarrow 0}\left(\varepsilon^{\frac{1}{2}-\frac{3}{2} \beta} P_{i}^{\varepsilon} \mathrm{e}^{-\mathrm{i} \frac{t}{\varepsilon} H^{\varepsilon}}-\mathrm{i} \int_{0}^{t} \mathrm{e}^{-\mathrm{i} \frac{t-s}{\varepsilon} H_{i, \text { field }}} \mathcal{T}_{j \rightarrow i} \mathrm{e}^{-\mathrm{i} \frac{s}{\varepsilon} H_{j, \text { field }}^{\varepsilon}} \mathrm{d} s\right)\left(P_{j}^{\varepsilon} \otimes Q_{0}\right) \mathbf{1}_{E}\left(H^{\varepsilon}\right)=0
$$

in the norm of bounded operators and uniformly on bounded time intervals. Here

$$
\mathcal{T}_{j \rightarrow i}=\frac{\mathrm{i} \delta}{2 \pi \varepsilon^{\frac{1}{2}}} \sum_{\lambda=1,2} \int_{|k|<\Lambda_{0}} \frac{e_{\lambda}(k) \cdot D_{i j}(x) \Delta_{E}(x)}{\sqrt{|k|}\left(|k|-\Delta_{E}(x)+\mathrm{i} \delta\right)}\left|\varphi_{i}(x)\right\rangle\left\langle\varphi_{j}(x)\right| \otimes a^{*}(k, \lambda) \mathrm{d} k
$$

with $\delta=\varepsilon^{\frac{1}{2}-\left(\beta-\frac{5}{6}\right) / 5}, \Delta_{E}(x):=E_{j}(x)-E_{i}(x)$ and $D_{i j}(x):=\sum_{\ell=1}^{r}\left\langle\varphi_{i}(x) \mid y_{\ell} \varphi_{j}(x)\right\rangle_{\mathcal{H}_{\mathrm{el}}}$.

In Theorem 2 we will show that the integral in (29) is of order one (with a norm independent of the precise choice for $\delta(\varepsilon)$ ), although this is not obvious from the prefactor and the norm of $\mathcal{T}_{j \rightarrow i}$. However, this observation justifies the interpretation of the integral as the leading order piece of the wave function that makes a transition from level $j$ to level $i$.

Proof. (of Theorem 1) In order to interchange energy cutoffs with unitary groups it turns out useful, that replacing energy cutoffs in terms of $H_{0}^{\varepsilon}$ by cutoffs in terms of $H^{\varepsilon}$ and vice versa only adds an error of order $\varepsilon^{\frac{3}{2} \beta}$. More precisely, the graph norms induced by $H_{0}^{\varepsilon}$ and $H^{\varepsilon}$ are equivalent and we have that

$$
\left\|\tilde{\chi}\left(H^{\varepsilon}\right)-\tilde{\chi}\left(H_{0}^{\varepsilon}\right)\right\|_{\mathcal{L}\left(\mathcal{H}, D_{0}\right)}=\mathcal{O}\left(\varepsilon^{\frac{3}{2} \beta}\right) .
$$

Both claims follow from the following lemma.

Lemma 3. Let $\left(H_{0}, D\left(H_{0}\right)\right)$ be self-adjoint and equip $D:=D\left(H_{0}\right)$ with the graph norm $\|\cdot\|_{D_{0}}$. If $A \in \mathcal{L}(D, \mathcal{H})$ satisfies

$$
\|A\|_{\mathcal{L}(D, \mathcal{H})} \leq \delta<1
$$

then $H=H_{0}+A$ is self-adjoint on $D(H)=D$ and the graph-norm $\|\cdot\|_{D_{H}}$ induced by $H$ on $D$ is equivalent to $\|\cdot\|_{D_{0}}$. More precisely, for $\psi \in D$

$$
(1-\delta)\|\psi\|_{D_{0}} \leq\|\psi\|_{D_{H}} \leq(1+\delta)\|\psi\|_{D_{0}} .
$$

Moreover, for any $\tilde{\chi} \in C_{0}^{\infty}(\mathbb{R})$ there is a constant $C<\infty$ (depending only on $\tilde{\chi}$, but not on $H, A$ or $\delta$ ) such that

$$
\left\|\tilde{\chi}\left(H_{0}\right)-\tilde{\chi}(H)\right\|_{\mathcal{L}(\mathcal{H}, D)} \leq C \delta
$$


As another useful consequence of this lemma we note that the full unitary group is uniformly bounded in $\mathcal{L}\left(D_{0}\right)$, i.e.

$$
\left\|\mathrm{e}^{-\mathrm{i} \frac{\underline{t}}{\varepsilon} H^{\varepsilon}}\right\|_{\mathcal{L}\left(D_{0}\right)} \leq C\left\|\mathrm{e}^{-\mathrm{i} \frac{\underline{t}}{\varepsilon} H^{\varepsilon}}\right\|_{\mathcal{L}\left(D_{H^{\varepsilon}}\right)}=C,
$$

where $D_{H^{\varepsilon}}$ denotes $D_{0}$ equipped with the graph norm of $H^{\varepsilon}$.

We now fix some $\tilde{\chi} \in C_{0}^{\infty}$ with $\tilde{\chi} \mathbf{1}_{E}=\mathbf{1}_{E}$ and $\tilde{\chi} \mathbf{1}_{E+\frac{1}{2}}=\tilde{\chi}$ and abbreviate $P_{j, \mathrm{v}}^{\varepsilon}:=$ $P_{j \text {,vac }}^{\varepsilon}$. Then (using Lemma 2 from now on implicitly)

$$
\begin{aligned}
P_{i}^{\varepsilon} P_{j, \mathrm{v}}^{\varepsilon} \tilde{\chi}\left(H^{\varepsilon}\right) \stackrel{26}{=} P_{i}^{\varepsilon} P_{j}^{\varepsilon} \otimes Q_{0} \tilde{\chi}\left(H^{\varepsilon}\right)+\mathcal{O}\left(\varepsilon^{\frac{3}{2} \beta} \delta^{-\frac{1}{2}}\right) \\
\stackrel{311}{=} P_{i}^{\varepsilon} P_{j}^{\varepsilon} \otimes Q_{0} \tilde{\chi}\left(H_{0}^{\varepsilon}\right)+\mathcal{O}\left(\varepsilon^{\frac{3}{2} \beta}\left(1+\delta^{-\frac{1}{2}}\right)\right) \stackrel{17}{=} \mathcal{O}\left(\varepsilon^{\frac{3}{2} \beta} \delta^{-\frac{1}{2}}\right)
\end{aligned}
$$

and, hence,

$$
P_{i}^{\varepsilon} P_{j, \mathrm{v}}^{\varepsilon} \mathrm{e}^{-\mathrm{i} \frac{t}{\varepsilon} H^{\varepsilon}} P_{j, \mathrm{v}}^{\varepsilon} \tilde{\chi}\left(H^{\varepsilon}\right) \stackrel{255}{=} P_{i}^{\varepsilon} P_{j, \mathrm{v}}^{\varepsilon} \tilde{\chi}\left(H^{\varepsilon}\right) \mathrm{e}^{-\mathrm{i} \frac{t}{\varepsilon} H^{\varepsilon}} P_{j, \mathrm{v}}^{\varepsilon}+\mathcal{O}\left(\varepsilon^{\frac{3}{2} \beta} \delta^{\frac{1}{2}}\right)=\mathcal{O}\left(\varepsilon^{\frac{3}{2} \beta} \delta^{-\frac{1}{2}}\right) .
$$

In the following computation we make explicit the size of the error term in the line where it first appears and collect all of them only in the final line. We use (28), (32) and $P_{j, \mathrm{v}}^{\varepsilon} \in \mathcal{L}\left(D_{0}\right)$ throughout without noting it explicitly. 


$$
\begin{aligned}
& P_{i}^{\varepsilon} \mathrm{e}^{-\mathrm{i} \frac{t}{\varepsilon} H^{\varepsilon}} P_{j}^{\varepsilon} \otimes Q_{0} \mathbf{1}_{E}\left(H^{\varepsilon}\right) \stackrel{26}{=} P_{i}^{\varepsilon} \mathrm{e}^{-\mathrm{i} \frac{t}{\varepsilon} H^{\varepsilon}} P_{j, \mathrm{v}}^{\varepsilon} \tilde{\chi}\left(H^{\varepsilon}\right) \mathbf{1}_{E}\left(H^{\varepsilon}\right)+\mathcal{O}\left(\varepsilon^{\frac{3}{2} \beta} \delta^{-\frac{1}{2}}\right) \\
& \stackrel{33}{=} P_{i}^{\varepsilon}\left[\mathrm{e}^{-\mathrm{i} \frac{t}{\varepsilon} H^{\varepsilon}}, P_{j, \mathrm{v}}^{\varepsilon}\right] P_{j, \mathrm{v}}^{\varepsilon} \tilde{\chi}\left(H^{\varepsilon}\right) \mathbf{1}_{E}\left(H^{\varepsilon}\right)+\mathcal{O}\left(\varepsilon^{\frac{3}{2} \beta} \delta^{-\frac{1}{2}}\right) \\
& \stackrel{25}{=} P_{i}^{\varepsilon}\left[\mathrm{e}^{-\mathrm{i} \frac{t}{\varepsilon} H^{\varepsilon}}, P_{j, \mathrm{v}}^{\varepsilon}\right] \tilde{\chi}\left(H^{\varepsilon}\right) P_{j, \mathrm{v}}^{\varepsilon} \mathbf{1}_{E}\left(H^{\varepsilon}\right)+\mathcal{O}\left(\varepsilon^{\frac{3}{2} \beta} \delta^{\frac{1}{2}}\right) \\
& =\quad-\frac{\mathrm{i}}{\varepsilon} \int_{0}^{t} P_{i}^{\varepsilon} \mathrm{e}^{-\mathrm{i} \frac{t-s}{\varepsilon} H^{\varepsilon}}\left[H^{\varepsilon}, P_{j, \mathrm{v}}^{\varepsilon}\right] \tilde{\chi}\left(H^{\varepsilon}\right) \mathrm{e}^{-\mathrm{i} \frac{s}{\varepsilon} H^{\varepsilon}} P_{j, \mathrm{v}}^{\varepsilon} \mathrm{d} s \mathbf{1}_{E}\left(H^{\varepsilon}\right) \\
& \text { 231, [31] }-\frac{\mathrm{i}}{\varepsilon} \int_{0}^{t} P_{i}^{\varepsilon} \mathrm{e}^{-\mathrm{i} \frac{t-s}{\varepsilon} H^{\varepsilon}}\left[H^{\varepsilon}, P_{j, \mathrm{v}}^{\varepsilon}\right] \tilde{\chi}\left(H_{0}^{\varepsilon}\right) \mathrm{e}^{-\mathrm{i} \frac{s}{\varepsilon} H^{\varepsilon}} P_{j, \mathrm{v}}^{\varepsilon} \mathrm{d} s \mathbf{1}_{E}\left(H^{\varepsilon}\right)+\mathcal{O}\left(\varepsilon^{\frac{3}{2} \beta}|t|\right) \\
& \stackrel{277}{=} \quad \mathrm{i} \varepsilon^{\frac{3}{2} \beta-1} \delta^{\frac{1}{2}} \int_{0}^{t} P_{i}^{\varepsilon} \mathrm{e}^{-\mathrm{i} \frac{t-s}{\varepsilon} H^{\varepsilon}} \mathcal{T}_{j} \tilde{\chi}\left(H_{0}^{\varepsilon}\right) \mathrm{e}^{-\mathrm{i} \frac{s}{\varepsilon} H^{\varepsilon}} P_{j, \mathrm{v}}^{\varepsilon} \mathrm{d} s \mathbf{1}_{E}\left(H^{\varepsilon}\right)+\mathcal{O}\left(\varepsilon^{\frac{3}{2} \beta}\left(1+\varepsilon \delta^{-\frac{5}{2}}\right)|t|\right) \\
& \stackrel{19}{=} \quad \mathrm{i} \varepsilon^{\frac{3}{2} \beta-1} \delta^{\frac{1}{2}} \int_{0}^{t} P_{i} \mathrm{e}^{-\mathrm{i} \frac{t-s}{\varepsilon} H^{\varepsilon}} \mathcal{T}_{j} \tilde{\chi}\left(H_{0}^{\varepsilon}\right) \mathrm{e}^{-\mathrm{i} \frac{s}{\varepsilon} H^{\varepsilon}} P_{j, \mathrm{v}}^{\varepsilon} \mathrm{d} s \mathbf{1}_{E}\left(H^{\varepsilon}\right)+\mathcal{O}\left(\varepsilon^{\frac{3}{2} \beta} \delta^{\frac{1}{2}}|t|\right) \\
& \stackrel{12}{=} \quad \mathrm{i} \varepsilon^{\frac{3}{2} \beta-1} \delta^{\frac{1}{2}} \int_{0}^{t} P_{i} \mathrm{e}^{-\mathrm{i} \frac{t-s}{\varepsilon} H_{i, \text { field }}} \mathcal{T}_{j} \tilde{\chi}\left(H_{0}^{\varepsilon}\right) \mathrm{e}^{-\mathrm{i} \frac{s}{\varepsilon} H^{\varepsilon}} P_{j, \mathrm{v}}^{\varepsilon} \mathrm{d} s \mathbf{1}_{E}\left(H^{\varepsilon}\right) \\
& +\mathcal{O}\left(\varepsilon^{3 \beta-2} \delta^{\frac{1}{2}}|t|^{2}+\varepsilon^{\frac{3}{2} \beta} \delta^{\frac{1}{2}}|t|\right)
\end{aligned}
$$

231, $=\mathrm{i} \varepsilon^{\frac{3}{2} \beta-1} \delta^{\frac{1}{2}} \int_{0}^{t} P_{i} \mathrm{e}^{-\mathrm{i} \frac{t-s}{\varepsilon} H_{i, \text { field }}^{\varepsilon}} \mathcal{T}_{j} \mathrm{e}^{-\mathrm{i} \frac{s}{\varepsilon} H^{\varepsilon}} P_{j, \mathrm{v}}^{\varepsilon} \tilde{\chi}\left(H_{0}^{\varepsilon}\right) \mathrm{d} s \mathbf{1}_{E}\left(H^{\varepsilon}\right)+\mathcal{O}\left(\varepsilon^{3 \beta-1} \delta^{\frac{1}{2}}|t|\right)$

$\stackrel{260,(19)}{=} \mathrm{i} \varepsilon^{\frac{3}{2} \beta-1} \delta^{\frac{1}{2}} \int_{0}^{t} P_{i} \mathrm{e}^{-\mathrm{i} \frac{t-s}{\varepsilon} H_{i, \text { field }}} \mathcal{T}_{j} \mathrm{e}^{-\mathrm{i} \frac{s}{\varepsilon} H^{\varepsilon}}\left(P_{j} \otimes Q_{0}\right) \tilde{\chi}\left(H_{0}^{\varepsilon}\right) \mathrm{d} s \mathbf{1}_{E}\left(H^{\varepsilon}\right)$

$$
+\mathcal{O}\left(\varepsilon^{3 \beta-1}|t|+\varepsilon^{\frac{3}{2} \beta} \delta^{\frac{1}{2}}|t|\right)
$$

$\stackrel{122}{=} \quad \mathrm{i} \varepsilon^{\frac{3}{2} \beta-1} \delta^{\frac{1}{2}} \int_{0}^{t} P_{i} \mathrm{e}^{-\mathrm{i} \frac{t-s}{\varepsilon} H_{i, \text { field }}^{\varepsilon}} \mathcal{T}_{j} \mathrm{e}^{-\mathrm{i} \frac{s}{\varepsilon} H_{j, \text { field }}^{\varepsilon}}\left(P_{j} \otimes Q_{0}\right) \tilde{\chi}\left(H_{0}^{\varepsilon}\right) \mathrm{d} s \mathbf{1}_{E}\left(H^{\varepsilon}\right)$

$$
\begin{gathered}
+\mathcal{O}\left(\varepsilon^{3 \beta-2} \delta^{\frac{1}{2}}|t|^{2}+\varepsilon^{\frac{3}{2} \beta} \delta^{\frac{1}{2}}|t|\right) \\
=\quad \mathrm{i} \varepsilon^{\frac{3}{2} \beta-1} \delta^{\frac{1}{2}} \int_{0}^{t} \mathrm{e}^{-\mathrm{i} \frac{t-s}{\varepsilon} H_{i, \text { field }}^{\varepsilon}} P_{i} \mathcal{T}_{j}\left(P_{j} \otimes Q_{0}\right) \mathrm{e}^{-\mathrm{i} \frac{s}{\varepsilon} H_{j, \text { field }}^{\varepsilon}} \mathrm{d} s \mathbf{1}_{E}\left(H^{\varepsilon}\right)
\end{gathered}
$$$$
+\mathcal{O}\left(\varepsilon^{3 \beta-2} \delta^{\frac{1}{2}}|t|^{2}+\varepsilon^{\frac{3}{2} \beta}\left(1+\varepsilon \delta^{-\frac{5}{2}}\right)|t|+\varepsilon^{\frac{3}{2} \beta} \delta^{-\frac{1}{2}}\right) .
$$

For our choice of $\delta=\varepsilon^{\frac{1}{2}-\left(\beta-\frac{5}{6}\right) / 5}$ the error term is $o\left(\varepsilon^{\frac{3}{2} \beta-\frac{1}{2}}\right)$ and we can neglect it in the following.

By using that $\left\|\left[\varepsilon \nabla_{x}, P_{j}\right]\right\|=\mathcal{O}(\varepsilon), P_{i} P_{j}=0, T_{j}^{*} Q_{0}=0, \nabla T_{j}^{*} Q_{0}=0$ and $\left\|\nabla T_{j}\right\|=$ $\mathcal{O}\left(\delta^{-\frac{3}{2}}\right)$, we see that

$$
\begin{aligned}
& \varepsilon^{-\frac{1}{2}} \delta^{\frac{1}{2}} P_{i} \mathcal{T}_{j}\left(P_{j} \otimes Q_{0}\right)= \\
& \quad=P_{i}\left(\mathrm{i} \varepsilon^{-\frac{1}{2}} \delta\left(T_{j}+T_{j}^{*}\right)+2 \varepsilon^{\frac{1}{2}}\left(\nabla T_{j}+\nabla T_{j}^{*}\right) \cdot \varepsilon \nabla_{x}\right)\left(P_{j} \otimes Q_{0}\right) \\
& \quad=\mathrm{i} \varepsilon^{-\frac{1}{2}} \delta P_{i} T_{j}\left(P_{j} \otimes Q_{0}\right)+2 \varepsilon^{\frac{1}{2}} P_{i} \nabla T_{j}\left(P_{j} \otimes Q_{0}\right) \cdot \varepsilon \nabla_{x}+\mathcal{O}\left(\varepsilon^{\frac{3}{2}} \delta^{-\frac{3}{2}}\right) .
\end{aligned}
$$


Thus the corresponding replacement in the integrand contributes an error of order $\mathcal{O}\left(\varepsilon^{\frac{3}{2} \beta+\frac{1}{2}} \delta^{-\frac{3}{2}}|t|\right)$. Since $\nabla_{x} P_{i}$ and $\nabla_{x} P_{j} \otimes Q_{0}$ are bounded independently of $\varepsilon$ and, since

$$
\left\|\left(H_{\mathrm{f}}+H_{\mathrm{el}}(x)-E_{j}(x)+\mathrm{i} \delta\right)^{-1}\right\| \leq \delta^{-1}
$$

we have

$$
\begin{aligned}
\varepsilon^{\frac{1}{2}} & P_{i} \nabla T_{j}\left(P_{j} \otimes Q_{0}\right) \\
& =-\varepsilon^{\frac{1}{2}} P_{i} \nabla\left(\left(H_{\mathrm{f}}+H_{\mathrm{el}}(x)-E_{j}(x)+\mathrm{i} \delta\right)^{-1} H_{1}^{\varepsilon}\left(P_{j} \otimes Q_{0}\right)\right)\left(P_{j} \otimes Q_{0}\right) \\
& =-\varepsilon^{\frac{1}{2}} \nabla\left(P_{i}(x)\left(H_{\mathrm{f}}+H_{\mathrm{el}}(x)-E_{j}(x)+\mathrm{i} \delta\right)^{-1} H_{1}^{\varepsilon}\right)\left(P_{j} \otimes Q_{0}\right)+\mathcal{O}\left(\varepsilon^{\frac{1}{2}} \delta^{-1}\right) \\
& =-\varepsilon^{\frac{1}{2}} P_{i} \nabla\left(\left(H_{\mathrm{f}}+E_{i}(x)-E_{j}(x)+\mathrm{i} \delta\right)^{-1} H_{1}^{\varepsilon}\right)\left(P_{j} \otimes Q_{0}\right)+\mathcal{O}\left(\varepsilon^{\frac{1}{2}} \delta^{-1}\right) \\
& =\varepsilon^{\frac{1}{2}} P_{i}\left(\nabla E_{i}(x)-\nabla E_{j}(x)\right)\left(H_{\mathrm{f}}+E_{i}(x)-E_{j}(x)+\mathrm{i} \delta\right)^{-2} H_{1}^{\varepsilon}\left(P_{j} \otimes Q_{0}\right)+\mathcal{O}\left(\varepsilon^{\frac{1}{2}} \delta^{-1}\right)
\end{aligned}
$$

in the norm of bounded operators. Recall that

$$
H_{1}^{\varepsilon}=-4 \sqrt{\pi} \sum_{\ell=1}^{r} A\left(\varepsilon^{\beta} y_{\ell}\right) \cdot p_{\ell, y}
$$

and thus on the one particle sector of Fock space, abbreviating $\hat{\rho}(k):=(2 \pi)^{-\frac{3}{2}} \mathbf{1}_{\left[0, \Lambda_{0}\right]}(k)$,

$$
\begin{aligned}
& P_{i} H_{1}^{\varepsilon}\left(P_{j} \otimes Q_{0}\right)(x, k, \lambda)= \\
& =-4 \sqrt{\pi} \sum_{\ell=1}^{r} \frac{\hat{\rho}(k)}{\sqrt{2|k|}}\left|\varphi_{i}(x)\right\rangle\left\langle\varphi_{i}(x), \mathrm{e}^{-\mathrm{i} \varepsilon^{\beta} k \cdot y_{\ell}} e_{\lambda}(k) \cdot \nabla_{y_{\ell}} \varphi_{j}(x)\right\rangle_{\mathcal{H}_{\mathrm{el}}}\left\langle\varphi_{j}(x)\right| \\
& =-4 \sqrt{\pi} \sum_{\ell=1}^{r} \frac{\hat{\rho}(k)}{\sqrt{2|k|}}\left|\varphi_{i}(x)\right\rangle\left\langle\varphi_{i}(x), e_{\lambda}(k) \cdot \nabla_{y_{\ell}} \varphi_{j}(x)\right\rangle_{\mathcal{H}_{\mathrm{el}}}\left\langle\varphi_{j}(x)\right|+\mathcal{O}\left(\varepsilon^{\beta}\right) \\
& =2 \sqrt{\pi} \sum_{\ell=1}^{r} \frac{\hat{\rho}(k)}{\sqrt{2|k|}}\left|\varphi_{i}(x)\right\rangle e_{\lambda}(k) \cdot\left\langle\varphi_{i}(x),\left[H_{\mathrm{el}}(x), y_{\ell}\right] \varphi_{j}(x)\right\rangle_{\mathcal{H}_{\mathrm{el}}}\left\langle\varphi_{j}(x)\right|+\mathcal{O}\left(\varepsilon^{\beta}\right) \\
& =2 \sqrt{\pi}\left(E_{i}(x)-E_{j}(x)\right) \frac{\hat{\rho}(k)}{\sqrt{2|k|}} e_{\lambda}(k) \cdot \sum_{\ell=1}^{r}\left\langle\varphi_{i}(x), y_{\ell} \varphi_{j}(x)\right\rangle_{\mathcal{H}_{\mathrm{el}}}\left|\varphi_{i}(x)\right\rangle\left\langle\varphi_{j}(x)\right|+\mathcal{O}\left(\varepsilon^{\beta}\right) \\
& =: \quad \sqrt{2 \pi}\left(E_{i}(x)-E_{j}(x)\right) \frac{\hat{\rho}(k)}{\sqrt{|k|}} e_{\lambda}(k) \cdot D_{i j}(x)\left|\varphi_{i}(x)\right\rangle\left\langle\varphi_{j}(x)\right|+\mathcal{O}\left(\varepsilon^{\beta}\right) .
\end{aligned}
$$

Collecting the previous observations, we showed (29) with a transition operator given by

$$
\begin{aligned}
\tilde{\mathcal{T}}_{j \rightarrow i}= & \frac{\mathrm{i} \delta}{2 \pi \varepsilon^{\frac{1}{2}}} \sum_{\lambda=1,2} \int_{|k|<\Lambda_{0}} \frac{e_{\lambda}(k) \cdot D_{i j}(x) \Delta_{E}(x)}{\sqrt{|k|}\left(|k|-\Delta_{E}(x)+\mathrm{i} \delta\right)} \times \\
& \left(1+\frac{2 \mathrm{i} \varepsilon \nabla_{x} \Delta_{E}(x)}{\delta \sqrt{|k|}\left(|k|-\Delta_{E}(x)+\mathrm{i} \delta\right)} \cdot\left(-\mathrm{i} \varepsilon \nabla_{x}\right)\right) \times \\
& \left|\varphi_{i}(x)\right\rangle\left\langle\varphi_{j}(x)\right| \otimes a^{*}(k) \mathrm{d} k+\mathcal{O}\left(\varepsilon^{\beta-\frac{1}{2}}+\varepsilon^{\frac{1}{2}} \delta^{-1}\right) \\
=: & t_{1}+t_{2}+\mathcal{O}\left(\varepsilon^{\beta-\frac{1}{2}}+\varepsilon^{\frac{1}{2}} \delta^{-1}\right) .
\end{aligned}
$$


The error term is $o(1)$ and we can neglect it. While the norm of $t_{2}$ is of order $\left(\frac{\varepsilon}{\delta^{3}}\right)^{\frac{1}{2}}$ and thus slightly smaller than that of $t_{1}$, which is of order $\left(\frac{\delta}{\varepsilon}\right)^{\frac{1}{2}}$, both grow as $\varepsilon \rightarrow 0$. In order to show that the contribution of $t_{1}$ to the integral in 29 is of order one and that of $t_{2}$ is strictly smaller, one has to perform the time integration and use cancellations due to oscillations. In order not to duplicate the corresponding arguments, we skip the proof that the contribution of $t_{2}$ is negligible at this point and comment on it instead after the proof of Theorem 2. At that point we will have introduced the necessary machinery in order to explain the argument.

\subsection{Spontaneous emission: rate of decay}

In order to obtain an explicit formula for the leading order rate of spontaneous decay from $\operatorname{Ran} P_{j}^{\varepsilon}$ to $\operatorname{Ran} P_{i}^{\varepsilon}$ we evaluate the norm of the leading order wave function in Theorem 1.

Theorem 2 (Probability for spontaneous decay). Under the same hypotheses as in Theorem 1 for

$$
\Psi=\psi \otimes \Omega \in\left(P_{j}^{\varepsilon} \otimes Q_{0}\right) \mathbf{1}_{E}\left(H^{\varepsilon}\right) \mathcal{H}
$$

it holds that

$$
\lim _{\varepsilon \rightarrow 0}\left(\varepsilon^{1-3 \beta}\left\|P_{i}^{\varepsilon} \mathrm{e}^{-\mathrm{i} \frac{t}{\varepsilon} H^{\varepsilon}} \Psi\right\|_{\mathcal{H}}^{2}-\int_{0}^{t} \frac{4}{3}\left\|\left|D_{i j}\right| \Delta_{E}^{3 / 2} \mathrm{e}^{-\mathrm{i} \frac{s}{\varepsilon} H_{j}^{\varepsilon}} P_{j} \psi\right\|_{\mathcal{H}_{\mathrm{mol}}}^{2} \mathrm{~d} s\right)=0
$$

uniformly on compact time intervals and uniformly in $\psi$.

Before we come to the proof, we collect some remarks on the result:

1. Note that the subtracted term in $(36)$ is of order 1 and therefore the same is true for $\varepsilon^{1-3 \beta}\left\|P_{i}^{\varepsilon} \mathrm{e}^{-\mathrm{i} \frac{t}{\varepsilon} H^{\varepsilon}} \Psi\right\|^{2}$. Now recall that $\varepsilon^{\beta}$ is equal to the coupling constant $\alpha$ by choice of $\beta$. So $\left\|P_{i}^{\varepsilon} \mathrm{e}^{-\mathrm{i} \frac{t}{\varepsilon} H^{\varepsilon}} \Psi\right\|^{2}$ is proportional to $\alpha^{3}$ and grows linearly in time. Observing that in our units time is scaled with $\alpha^{2}$, we see that Theorem 2 is the generalization to molecules of the physics textbook result that for atoms the decay rate is $\frac{4}{3} \alpha^{5}\left|D_{i j}\right|^{2} \Delta_{E}^{3}$.

2. For $\beta<1$ we may replace $P_{i}^{\varepsilon}$ and $P_{j}^{\varepsilon}$ by $P_{i}$ and $P_{j}$ respectively in the theorem because $P_{k}^{\varepsilon}-P_{k}=\mathcal{O}(\varepsilon)$ for $k=i, j$ and $\varepsilon^{\frac{3}{2} \beta-\frac{1}{2}}>\varepsilon$.

3. Practically the result (36) means that the decay rate can be computed at leading order within the Born-Oppenheimer approximation: while $\psi \in \mathcal{H}_{\text {mol }}$ still contains the electronic degrees of freedom, $P_{j} \psi$ is of the form $\phi(x) \varphi_{j}(x, y)$ and therefore

$$
\left(\mathrm{e}^{-\mathrm{i} \frac{s}{\varepsilon} H_{j}^{\varepsilon}} P_{j} \psi\right)(x, y)=\left(\mathrm{e}^{-\mathrm{i} \frac{s}{\varepsilon} h_{j}^{\varepsilon}} \phi\right)(x) \varphi_{j}(x, y),
$$

where the effective Born-Oppenheimer Hamiltonian is just

$$
h_{\mathrm{el}}^{\varepsilon}=\varepsilon^{2}\left(-\mathrm{i} \nabla_{x}+\mathcal{A}_{j}(x)\right)^{2}+E_{j}(x)+\mathcal{O}\left(\varepsilon^{2}\right),
$$


where $\mathcal{A}_{j}(x):=\mathrm{i}\left\langle\varphi_{j}(x), \nabla \varphi_{j}(x)\right\rangle_{\mathcal{H}_{\mathrm{el}}}$ the connection coefficient of the Berry-connection $\nabla_{x}^{j}:=P_{j} \nabla_{x} P_{j}$. Hence the decay rate can be written as

$$
\frac{4}{3}\left\|\left|D_{i j}\right| \Delta_{E}^{3 / 2} \mathrm{e}^{-\mathrm{i} \frac{s}{\varepsilon} H_{j}^{\varepsilon}} P_{j} \psi\right\|_{\mathcal{H}_{\text {mol }}}^{2}=\frac{4}{3}\left\|\left|D_{i j}\right| \Delta_{E}^{3 / 2} \mathrm{e}^{-\mathrm{i} \frac{s}{\varepsilon} h_{j}^{\varepsilon}} \phi\right\|_{\mathcal{H}_{\text {nuc }}}^{2}
$$

and one only needs to solve an effective Schrödinger equation for the nuclei.

Proof. (of Theorem 2) According to Theorem 1 we have that

$$
\lim _{\varepsilon \rightarrow 0}\left(\varepsilon^{1-3 \beta}\left\|P_{i}^{\varepsilon} \mathrm{e}^{-\mathrm{i} \frac{t}{\varepsilon} H^{\varepsilon}} \Psi\right\|^{2}-\Theta_{i j}(t)\right)=0
$$

with

$$
\Theta_{i j}(t):=\left\|\int_{0}^{t} \mathrm{~d} r \mathrm{e}^{\mathrm{i} \frac{r}{\varepsilon} H_{i, \mathrm{f}}^{\varepsilon}} \mathcal{T}_{j \rightarrow i} \mathrm{e}^{-\mathrm{i} \frac{r}{\varepsilon} H_{j, \mathrm{f}}^{\varepsilon}} \Psi\right\|^{2}
$$

and we need to show that $\lim _{\varepsilon \rightarrow 0}\left(\Theta_{i j}(t)-\int_{0}^{t} \frac{4}{3}\left\|\left|D_{i j}\right| \Delta_{E}^{3 / 2} \mathrm{e}^{-\mathrm{i} \frac{s}{\varepsilon} H_{j}^{\varepsilon}} \psi\right\|^{2} \mathrm{~d} s\right)=0$. First note that because of the explicit form $(30)$ of $\mathcal{T}_{j \rightarrow i}$ this state lives only the one-particle sector of Fock space. Writing $k=\omega|k|$, the only dependence on the angular variable $\omega$ appears in the polarization vectors $e_{\lambda}(\omega)$. Using that for any symmetric $3 \times 3$-matrix $A$ it holds that

$$
\sum_{\lambda=1,2} \int_{S^{2}} \mathrm{~d} \omega\left\langle e_{\lambda}(\omega), A e_{\lambda}(\omega)\right\rangle=\frac{8 \pi}{3} \operatorname{tr} A
$$

one can perform the angular integration in 37 and obtains a factor $\frac{8 \pi}{3}$. However, to not overburden notation, we will make this explicit only later on. First we rewrite (37) as

$$
\Theta_{i j}(t)=\varepsilon^{2} \int_{0}^{\frac{t}{\varepsilon}} \mathrm{d} s \int_{-a(s)}^{a(s)} \mathrm{d} s^{\prime} \underbrace{\left\langle\Psi, \mathrm{e}^{\mathrm{i}\left(s+\frac{s^{\prime}}{2}\right) H_{j, \mathrm{f}}^{\varepsilon}} \mathcal{T}_{j \rightarrow i}^{*} \mathrm{e}^{-\mathrm{i} s^{\prime} H_{i, \mathrm{f}}^{\varepsilon}} \mathcal{T}_{j \rightarrow i} \mathrm{e}^{-\mathrm{i}\left(s-\frac{s^{\prime}}{2}\right) H_{j, \mathrm{f}}^{\varepsilon} \Psi}\right\rangle}_{=: \tilde{I}\left(s, s^{\prime}\right)}
$$

where $s=\left(r+r^{\prime}\right) /(2 \varepsilon), s^{\prime}=\left(r-r^{\prime}\right) / \varepsilon, a(s):=\min \left\{2 s, 2\left(\frac{t}{\varepsilon}-s\right)\right\}$. Let

$$
\tilde{H}_{j}:=P_{j}\left(-\varepsilon^{2} \Delta_{x}\right) P_{j}+P_{i}\left(-\varepsilon^{2} \Delta_{x}\right) P_{i}+E_{j}
$$

and

$$
\tilde{H}_{i}:=P_{j}\left(-\varepsilon^{2} \Delta_{x}\right) P_{j}+P_{i}\left(-\varepsilon^{2} \Delta_{x}\right) P_{i}+E_{i}
$$

which satisfy

$$
P_{j} \tilde{H}_{j}=\tilde{H}_{j} P_{j}=P_{j} H_{j}^{\varepsilon} P_{j}, \quad P_{i} \tilde{H}_{i}=\tilde{H}_{i} P_{i}=P_{i} H_{i}^{\varepsilon} P_{i} \quad \text { and } \quad \tilde{H}_{j}-\tilde{H}_{i}=\Delta .
$$

Then with $\mathcal{T}_{j \rightarrow i}=P_{i} \mathcal{T}_{j \rightarrow i} P_{j}$ and $\Psi=\psi \otimes \Omega$ we have that

$$
\begin{aligned}
\tilde{I}\left(s, s^{\prime}\right) & =\left\langle\Psi, \mathrm{e}^{\mathrm{i}\left(s+\frac{s^{\prime}}{2}\right) H_{j}^{\varepsilon}} P_{j} \mathcal{T}_{j \rightarrow i}^{*} P_{i} \mathrm{e}^{-\mathrm{i} s^{\prime} H_{i, \mathrm{f}}^{\varepsilon}} P_{i} \mathcal{T}_{j \rightarrow i} P_{j} \mathrm{e}^{-\mathrm{i}\left(s-\frac{s^{\prime}}{2}\right) H_{j}^{\varepsilon}} \Psi\right\rangle \\
& =\left\langle\Psi, \mathrm{e}^{\mathrm{i}\left(s+\frac{s^{\prime}}{2}\right) \tilde{H}_{j}} P_{j} \mathcal{T}_{j \rightarrow i}^{*} P_{i} \mathrm{e}^{-\mathrm{i} s^{\prime}\left(\tilde{H}_{i}+H_{\mathrm{f}}\right)} P_{i} \mathcal{T}_{j \rightarrow i} P_{j} \mathrm{e}^{-\mathrm{i}\left(s-\frac{s^{\prime}}{2}\right) \tilde{H}_{j}} \Psi\right\rangle .
\end{aligned}
$$


Making the angular integration explicit, we can thus replace $\tilde{I}\left(s, s^{\prime}\right)$ in 38 by

$$
\begin{gathered}
I\left(s, s^{\prime}\right)=\frac{8 \pi}{3} \frac{1}{(2 \pi)^{2}} \frac{\delta^{2}}{\varepsilon} \int_{0}^{\Lambda_{0}} \mathrm{~d} R R \mathrm{e}^{-\mathrm{i} s^{\prime} R}\left\langle\psi, \mathrm{e}^{\mathrm{i}\left(s+\frac{s^{\prime}}{2}\right) \tilde{H}_{j}} \frac{D^{*}(x) \Delta(x)}{R-\Delta(x)-\mathrm{i} \delta} \mathrm{e}^{-\mathrm{i}\left(s+\frac{s^{\prime}}{2}\right) \tilde{H}_{j}}\right. \\
\times \underbrace{\mathrm{i}^{\mathrm{i}\left(s+\frac{s^{\prime}}{2}\right) \tilde{H}_{j}} \mathrm{e}^{-\mathrm{i} s^{\prime}\left(\tilde{H}_{j}-\Delta(x)\right)} \mathrm{e}^{-\mathrm{i}\left(s-\frac{s^{\prime}}{2}\right) \tilde{H}_{j}}}_{=: U\left(s, s^{\prime}\right)} \mathrm{e}^{\mathrm{i}\left(s-\frac{s^{\prime}}{2}\right) \tilde{H}_{j}} \frac{D(x) \Delta(x)}{R-\Delta(x)+\mathrm{i} \delta} \mathrm{e}^{-\mathrm{i}\left(s-\frac{s^{\prime}}{2}\right) \tilde{H}_{j}} \psi\rangle \\
=\frac{2 \delta^{2}}{3 \pi \varepsilon} \int_{0}^{\Lambda_{0}} \mathrm{~d} R R \mathrm{e}^{-\mathrm{i} s^{\prime} R}\left\langle\psi, \frac{D^{*}\left(s+\frac{s^{\prime}}{2}\right) \Delta\left(s+\frac{s^{\prime}}{2}\right)}{R-\Delta\left(s+\frac{s^{\prime}}{2}\right)-\mathrm{i} \delta} U\left(s, s^{\prime}\right) \frac{D\left(s-\frac{s^{\prime}}{2}\right) \Delta\left(s-\frac{s^{\prime}}{2}\right)}{R-\Delta\left(s-\frac{s^{\prime}}{2}\right)+\mathrm{i} \delta} \psi\right\rangle .
\end{gathered}
$$

Here and in the following we denote for any operator $O$ the Heisenberg operator $\mathrm{e}^{\mathrm{i} s \tilde{H}_{j}} O \mathrm{e}^{-\mathrm{i} s \tilde{H}_{j}}$ by $O(s)$ and for better readability we abbreviate $R:=|k|$ and $D:=D_{i j}$. Moreover, we will still write out $\delta$ in the expressions and in most remainder estimates, but keep in mind that in the end we put $\delta=\varepsilon^{\frac{1}{2}-\left(\beta-\frac{5}{6}\right) / 5}$.

Next we show by a stationary phase argument that $I$ is small for large $s^{\prime}$. Integration by parts in

$$
I\left(s, s^{\prime}\right)=\frac{2 \delta^{2}}{3 \pi \varepsilon} \int_{0}^{\Lambda_{0}} \mathrm{~d} R R\left(\left(\frac{\mathrm{i}}{s^{\prime}} \frac{\mathrm{d}}{\mathrm{d} R}\right)^{l} \mathrm{e}^{-\mathrm{i} s^{\prime} R}\right) \frac{D^{*}\left(s+\frac{s^{\prime}}{2}\right) \Delta\left(s+\frac{s^{\prime}}{2}\right)}{R-\Delta\left(s+\frac{s^{\prime}}{2}\right)-\mathrm{i} \delta} U\left(s, s^{\prime}\right) \frac{D\left(s-\frac{s^{\prime}}{2}\right) \Delta\left(s-\frac{s^{\prime}}{2}\right)}{R-\Delta\left(s-\frac{s^{\prime}}{2}\right)+\mathrm{i} \delta},
$$

shows that

$$
I=\mathcal{O}\left(\delta^{-l} \varepsilon^{-1} \tau^{-l}+\delta^{2} \varepsilon^{-1} \tau^{-1}\right) \quad \text { for } \quad\left|s^{\prime}\right| \geq \tau \text { and } \tau \geq \delta^{-1} .
$$

Instead of giving the detailed computation we just mention that the boundary terms contain the operators

$$
\frac{1}{-\Delta\left(s \pm \frac{s^{\prime}}{2}\right) \pm \mathrm{i} \delta} \quad \text { and } \quad \frac{1}{\Lambda_{0}-\Delta\left(s \pm \frac{s^{\prime}}{2}\right) \pm \mathrm{i} \delta},
$$

which are all uniformly bounded, since $0<\Delta(x)<\Lambda_{0}$ uniformly in $x$. So the first boundary term, which is of order $\delta^{2} \varepsilon^{-1} \tau^{-1}$, is indeed the worst.

Next, for $\left|s^{\prime}\right| \leq \tau$ we expand the operators around $s^{\prime}=0$. Clearly

$$
\Delta\left(s \pm \frac{s^{\prime}}{2}\right)=\Delta(s) \pm \frac{\mathrm{i}}{2} \int_{0}^{s^{\prime}}\left[H_{j}^{\varepsilon}, \Delta\left(s \pm \frac{s^{\prime \prime}}{2}\right)\right] \mathrm{d} s^{\prime \prime}=\Delta(s)+\mathcal{O}(\varepsilon \tau)
$$

in $\mathcal{L}\left(D_{0}, \mathcal{H}\right)$ because the gradient of $\Delta$ is bounded independently of $\varepsilon$. The same is true for $\Delta$ replaced by $D$ because $\varphi_{i}$ and $\varphi_{j}$ as well as their derivatives with respect to $x$ decay exponentially in $y$ (the proof in WaTe is easily adapted to unbounded potentials whose derivatives with respect to $x$ are bounded). By using the so-called Strang splitting (see [JaLu]) we see that

$$
\begin{aligned}
U\left(s, s^{\prime}\right) & =\mathrm{e}^{\mathrm{i}\left(s+\frac{s^{\prime}}{2}\right) H_{j}^{\varepsilon}} \mathrm{e}^{-\mathrm{i} s^{\prime}\left(H_{j}^{\varepsilon}-\Delta(x)\right)} \mathrm{e}^{-\mathrm{i}\left(s-\frac{s^{\prime}}{2}\right) H_{j}^{\varepsilon}} \\
& =\mathrm{e}^{\mathrm{i}\left(s+\frac{s^{\prime}}{2}\right) H_{j}^{\varepsilon}} \mathrm{e}^{-\mathrm{i} \frac{s^{\prime}}{2} H_{j}^{\varepsilon}} \mathrm{e}^{\mathrm{i} s^{\prime} \Delta(x)} \mathrm{e}^{-\mathrm{i} \frac{s^{\prime}}{2} H_{j}^{\varepsilon}} \mathrm{e}^{-\mathrm{i}\left(s-\frac{s^{\prime}}{2}\right) H_{j}^{\varepsilon}}+\mathcal{O}\left(\tau^{3}\left\|\left[\Delta,\left[H_{j}^{\varepsilon}, \Delta\right]\right]\right\|\right) \\
& =\mathrm{e}^{\mathrm{i} s^{\prime} \Delta(s)}+\mathcal{O}\left(\varepsilon^{2} \tau^{3}\right)
\end{aligned}
$$


in $\mathcal{L}\left(D_{0}, \mathcal{H}\right)$. Plugging these expansions of $\Delta, D$, and $U$ into $I$, which is allowed because $\Psi \in D_{0}$ due to the energy cutoff and all operators involved are in $\mathcal{L}\left(D_{0}\right)$ with a norm bounded independently of $\varepsilon, \delta, s$ and $s^{\prime}$, we find that for $\left|s^{\prime}\right| \leq \tau$

$$
\begin{aligned}
I\left(s, s^{\prime}\right)= & \frac{2 \delta^{2}}{3 \pi \varepsilon} \int_{0}^{\Lambda_{0}} \mathrm{~d} R R \mathrm{e}^{-\mathrm{i} s^{\prime} R} \underbrace{\frac{D^{*}(s) \Delta(s)}{R-\Delta\left(s+\frac{s^{\prime}}{2}\right)-\mathrm{i} \delta} \mathrm{e}^{\mathrm{i} s^{\prime} \Delta(s)} \frac{D(s) \Delta(s)}{R-\Delta\left(s-\frac{s^{\prime}}{2}\right)+\mathrm{i} \delta}}_{=: J\left(s, s^{\prime}, R, \delta\right)} \\
& +\mathcal{O}\left(\tau+\varepsilon \tau^{3}\right) .
\end{aligned}
$$

While this is not small, note that the contribution of the error term to $\Theta_{i j}$ is of order

$$
\varepsilon^{2} \int_{0}^{\frac{t}{\varepsilon}} \mathrm{d} s \int_{-\tau}^{\tau} \mathrm{d} s^{\prime} \mathcal{O}\left(\tau+\varepsilon \tau^{3}\right)=\mathcal{O}\left(\varepsilon \tau^{2}+\varepsilon^{2} \tau^{4}\right),
$$

which is indeed small for our choice of $\tau=\varepsilon^{-\frac{1}{2}+\left(\beta-\frac{5}{6}\right) / 10}$.

When expanding the denominators in (41), we have to be more careful, since e.g.

$$
\begin{aligned}
& \frac{1}{R-\Delta\left(s+\frac{s^{\prime}}{2}\right)-\mathrm{i} \delta}-\frac{1}{R-\Delta(s)-\mathrm{i} \delta} \\
& \quad=\frac{1}{R-\Delta\left(s+\frac{s^{\prime}}{2}\right)-\mathrm{i} \delta}\left(\Delta\left(s+\frac{s^{\prime}}{2}\right)-\Delta(s)\right) \frac{1}{R-\Delta(s)-\mathrm{i} \delta}
\end{aligned}
$$

is only $\mathcal{O}\left(\frac{\varepsilon \tau}{\delta^{2}}\right)$ when naively estimating the norm, which yields a term of order $\mathcal{O}\left(\frac{\varepsilon \tau^{2}}{\delta}\right)$ to $\Theta_{i j}$. This will be large for our choice of $\delta(\varepsilon)$ and $\tau(\varepsilon)$ and thus we need a better estimate. For this we have to evaluate the integral explicitly. In order to prepare for the residue calculus, we first show that we can extend the $R$-integration to all of $\mathbb{R}$ with a negligible error. Adding the integral to $+\infty$ yields

$$
\begin{aligned}
\frac{2 \delta^{2}}{3 \pi \varepsilon} \int_{-\tau}^{\tau} \varepsilon \mathrm{d} s^{\prime} & \lim _{\rho \rightarrow \infty} \int_{\Lambda_{0}}^{\rho} \mathrm{d} R R \mathrm{e}^{-\mathrm{i} s^{\prime} R} J\left(s, s^{\prime}, R, \delta\right) \\
= & \frac{2 \delta^{2}}{3 \pi \varepsilon} \lim _{\rho \rightarrow \infty} \int_{-\tau}^{\tau} \varepsilon \mathrm{d} s^{\prime} \int_{\Lambda_{0}}^{\rho} \mathrm{d} R\left(\mathrm{i} \frac{\mathrm{d}}{\mathrm{d} s^{\prime}} \mathrm{e}^{-\mathrm{i} s^{\prime} R}\right) J\left(s, s^{\prime}, R, \delta\right) \\
= & -\frac{2 \mathrm{i} \delta^{2}}{3 \pi \varepsilon} \lim _{\rho \rightarrow \infty} \int_{-\tau}^{\tau} \varepsilon \mathrm{d} s^{\prime} \int_{\Lambda_{0}}^{\rho} \mathrm{d} R \mathrm{e}^{-\mathrm{i} s^{\prime} R} \frac{\mathrm{d}}{\mathrm{d} s^{\prime}} J\left(s, s^{\prime}, R, \delta\right) \\
& +\frac{2 \mathrm{i} \delta^{2}}{3 \pi \varepsilon} \varepsilon \lim _{\rho \rightarrow \infty} \int_{\Lambda_{0}}^{\rho} \mathrm{d} R\left(\mathrm{e}^{-\mathrm{i} \tau R} J(s, \tau, R, \delta)-\mathrm{e}^{\mathrm{i} \tau R} J(s,-\tau, R, \delta)\right) \\
= & \mathcal{O}\left(\delta^{2} \tau+\delta^{2}\right),
\end{aligned}
$$

since $0<\Delta(x)<\Lambda_{0}$ uniformly in $x$ implies that $J\left(s, s^{\prime}, R, \delta\right)$ and $\frac{\mathrm{d}}{\mathrm{d} s^{\prime}} J\left(s, s^{\prime}, R, \delta\right)$ are uniformly bounded by $\frac{1}{R^{2}}$ in $\mathcal{L}\left(D_{0}, \mathcal{H}\right)$ for $R \geq \Lambda_{0}$ and analogously for $R \leq 0$. Thus we can integrate from $-\infty$ to $\infty$ in $R$ while adding an error of order $\delta^{2} \tau$ to $\Theta_{i j}$.

Now we want to replace $J\left(s, s^{\prime}, R, \delta\right)$ in (41) by

$$
\tilde{J}\left(s, s^{\prime}, R, \delta\right):=\frac{D^{*}(s) \Delta(s)}{R-\Delta(s)-\mathrm{i} \delta} \mathrm{e}^{\mathrm{i} s^{\prime} \Delta(s)} \frac{D(s) \Delta(s)}{R-\Delta(s)+\mathrm{i} \delta} .
$$


One of the two terms appearing in the difference is

$$
\frac{2 \delta^{2}}{3 \pi \varepsilon} \lim _{\rho \rightarrow \infty} \int_{-\rho}^{\rho} \mathrm{d} R R \mathrm{e}^{-\mathrm{i} s^{\prime} R}\left(\frac{1}{R-\Delta_{+}-\mathrm{i} \delta}-\frac{1}{R-\Delta_{0}-\mathrm{i} \delta}\right) f(s) \frac{1}{R-\Delta_{-}+\mathrm{i} \delta},
$$

where we abbreviate

$$
\Delta_{ \pm}:=\Delta\left(s \pm \frac{s^{\prime}}{2}\right), \quad \Delta_{0}:=\Delta(s) \quad \text { and } \quad f(s):=D^{*}(s) D(s) \Delta(s)^{2} \mathrm{e}^{\mathrm{i} s^{\prime} \Delta(s)} .
$$

To show that this term (and analogously the other one) gives only a negligible contribution to $\Theta_{i j}$, we use the residue calculus. For $s^{\prime}<0$ we need to close the contour in the upper complex plane. Writing the spectral representation of the self-adjoint operators $\Delta_{ \pm}$resp. $\Delta_{0}$ with spectrum contained in $\left(0, \Lambda_{0}\right)$ as

$$
\Delta_{ \pm, 0}=: \int_{0}^{\Lambda_{0}} \lambda \mathrm{d} P_{\lambda}^{ \pm, 0}
$$

the residue theorem yields for $s^{\prime}<0$

$$
\begin{aligned}
& \frac{2 \delta^{2}}{3 \pi \varepsilon} \lim _{\rho \rightarrow \infty} \int_{-\rho}^{\rho} \mathrm{d} R R \mathrm{e}^{-\mathrm{i} s^{\prime} R}\left(\frac{1}{R-\Delta_{+}-\mathrm{i} \delta}-\frac{1}{R-\Delta_{0}-\mathrm{i} \delta}\right) f(s) \frac{1}{R-\Delta_{-}+\mathrm{i} \delta} \\
& \quad=\frac{2 \delta^{2}}{3 \pi \varepsilon} \lim _{\rho \rightarrow \infty} \int_{0}^{\Lambda_{0}} \int_{-\rho}^{\rho} \mathrm{d} R R \mathrm{e}^{-\mathrm{i} s^{\prime} R}\left(\frac{\mathrm{d} P_{\lambda}^{+}}{R-\lambda-\mathrm{i} \delta}-\frac{\mathrm{d} P_{\lambda}^{0}}{R-\lambda-\mathrm{i} \delta}\right) f(s) \frac{1}{R-\Delta_{-}+\mathrm{i} \delta} \\
& =\frac{2 \delta^{2}}{3 \pi \varepsilon} \int_{0}^{\Lambda_{0}} \lambda \mathrm{e}^{-\mathrm{i} s^{\prime}(\lambda+\mathrm{i} \delta)}\left(\mathrm{d} P_{\lambda}^{+}-\mathrm{d} P_{\lambda}^{0}\right) f(s) \frac{1}{\lambda-\Delta_{-}+2 \mathrm{i} \delta}+\mathcal{O}\left(\frac{\delta^{2}}{\varepsilon}\right)=\quad(*) .
\end{aligned}
$$

According to 42 ) we can replace $\Delta_{-}$by $\Delta_{0}$ in the resolvent at the price of a term of order $\frac{\delta^{2}}{\varepsilon} \cdot \frac{\varepsilon \tau}{\delta^{2}}=\tau$. Then one can commute the resolvent with $f(s)$ and afterwards replace by the same reasoning $\Delta_{0}$ by $\Delta_{+}$for the first summand. Now one can integrate the spectral measures explicitly again and obtains

$$
\begin{aligned}
(*) & =\frac{2 \delta^{2}}{3 \pi \varepsilon} \int_{0}^{\Lambda_{0}} \lambda \mathrm{e}^{-\mathrm{i} s^{\prime}(\lambda+\mathrm{i} \delta)}\left(\frac{\mathrm{d} P_{\lambda}^{+}}{\lambda-\Delta_{+}+2 \mathrm{i} \delta}-\frac{\mathrm{d} P_{\lambda}^{0}}{\lambda-\Delta_{0}+2 \mathrm{i} \delta}\right) f(s)+\mathcal{O}\left(\frac{\delta^{2}}{\varepsilon}+\tau\right) \\
& =\frac{2 \delta^{2}}{3 \pi \varepsilon} \mathrm{e}^{-\left|s^{\prime}\right| \delta}\left(\frac{\mathrm{e}^{-\mathrm{i} s^{\prime} \Delta_{+}} \Delta_{+}}{2 \mathrm{i} \delta}-\frac{\mathrm{e}^{-\mathrm{i} s^{\prime} \Delta_{0}} \Delta_{0}}{2 \mathrm{i} \delta}\right) f(s)+\mathcal{O}\left(\frac{\delta^{2}}{\varepsilon}+\tau\right) \\
& =\mathcal{O}\left(\tau^{2} \delta+\frac{\delta^{2}}{\varepsilon}+\tau\right),
\end{aligned}
$$

where we used that by exactly the same reasoning as in 40 we have

$$
\mathrm{e}^{-\mathrm{i} s^{\prime} \Delta_{+}} \Delta_{+}-\mathrm{e}^{-\mathrm{i} s^{\prime} \Delta_{0}} \Delta_{0}=\mathcal{O}\left(\varepsilon \tau^{2}\right)
$$

for $\left|s^{\prime}\right| \leq \tau$. The additional factor of $\tau$ comes from the fact that derivatives of $\mathrm{e}^{\mathrm{i} s^{\prime} \Delta(x)}$ are of order $\left|s^{\prime}\right| \leq \tau$. After integration over $s$ and $s^{\prime}$ this adds an error of order $\varepsilon \tau^{3} \delta+\tau \delta^{2}+\varepsilon \tau^{2}$ 
to $\Theta_{i j}$. To estimate (44) for $s^{\prime}>0$, one closes the contour in the lower complex plane and proceeds along the same lines as above.

Let $a_{\tau}(s):=\min \{a(s), \tau\}$, then collecting once more all the estimates we obtain

$$
\begin{aligned}
\varepsilon^{2} \int_{0}^{\frac{t}{\varepsilon}} \mathrm{d} s \int_{-\frac{t}{\varepsilon}}^{\frac{t}{\varepsilon}} \mathrm{d} s^{\prime} I\left(s, s^{\prime}\right)-\frac{2 \delta^{2} \varepsilon}{3 \pi} \int_{0}^{\frac{t}{\varepsilon}} \mathrm{d} s \int_{-a_{\tau}(s)}^{a_{\tau}(s)} \mathrm{d} s^{\prime} \lim _{\rho \rightarrow \infty} \int_{-\rho}^{\rho} \mathrm{d} R R \mathrm{e}^{-\mathrm{i} s^{\prime} R} \tilde{J}\left(s, s^{\prime}, R, \delta\right) \\
\quad \varepsilon_{0}^{\frac{t}{\varepsilon}} \mathrm{d} s \int_{-\frac{t}{\varepsilon}}^{\frac{t}{\varepsilon}} \mathrm{d} s^{\prime} \mathcal{O}\left((\delta \tau)^{-l} \varepsilon^{-1}+\delta^{2} \varepsilon^{-1} \tau^{-1}\right) \\
+\varepsilon^{2} \int_{0}^{\frac{t}{\varepsilon}} \mathrm{d} s \int_{-\tau}^{\tau} \mathrm{d} s^{\prime} \mathcal{O}\left(\tau+\varepsilon \tau^{3}\right) \\
+\varepsilon \int_{0}^{\frac{t}{\varepsilon}} \mathrm{d} s \mathcal{O}\left(\delta^{2} \tau+\delta^{2}\right) \\
+43+\varepsilon^{2} \int_{0}^{\frac{t}{\varepsilon}} \mathrm{d} s \int_{-\tau}^{\tau} \mathrm{d} s^{\prime} \mathcal{O}\left(\tau^{2} \delta+\frac{\delta^{2}}{\varepsilon}+\tau\right) \\
=\mathcal{O}\left((\delta \tau)^{-l} \varepsilon^{-1}+\delta^{2} \varepsilon^{-1} \tau^{-1}+\delta^{2} \tau+\delta^{2}+\varepsilon \tau^{2}+\varepsilon^{2} \tau^{4}+\varepsilon \tau^{3} \delta\right) .
\end{aligned}
$$

Recall that $\delta=\varepsilon^{\frac{1}{2}-\left(\beta-\frac{5}{6}\right) / 5}$ and $\tau=\varepsilon^{-\frac{1}{2}+\left(\beta-\frac{5}{6}\right) / 10}$ and thus $\delta \tau=\varepsilon^{-\left(\beta-\frac{5}{6}\right) / 10} \gg 1$ for $\frac{5}{6}<\beta \leq \frac{4}{3}$. Then for $l$ big enough, the error is $o(1)$ for all $\beta$ with $\frac{5}{6}<\beta \leq \frac{4}{3}$.

Finally, we compute the main term using again the residue calculus. We close the integral depending on the sign of $s^{\prime}$ and get

$$
\begin{aligned}
& \frac{2 \delta^{2} \varepsilon}{3 \pi} \int_{0}^{\frac{t}{\varepsilon}} \mathrm{d} s \int_{-a_{\tau}(s)}^{a_{\tau}(s)} \mathrm{d} s^{\prime} \lim _{\rho \rightarrow \infty} \int_{-\rho}^{\rho} \mathrm{d} R R \mathrm{e}^{-\mathrm{i} s^{\prime} R} \tilde{J}\left(s, s^{\prime}, R, \delta\right)= \\
& =\frac{2 \delta^{2} \varepsilon}{3 \pi} \int_{0}^{\frac{t}{\varepsilon}} \mathrm{d} s \int_{-a_{\tau}(s)}^{a_{\tau}(s)} \mathrm{d} s^{\prime} \lim _{\rho \rightarrow \infty} \int_{-\rho}^{\rho} \mathrm{d} R R \mathrm{e}^{-\mathrm{i} s^{\prime} R} \frac{D^{*}(s) \Delta(s)}{R-\Delta(s)-\mathrm{i} \delta} \mathrm{e}^{\mathrm{i} s^{\prime} \Delta(s)} \frac{D(s) \Delta(s)}{R-\Delta(s)+\mathrm{i} \delta} \\
& =\frac{2 \delta \varepsilon}{3} \int_{0}^{\frac{t}{\varepsilon}} \mathrm{d} s \int_{-a_{\tau}(s)}^{a_{\tau}(s)} \mathrm{d} s^{\prime} \mathrm{e}^{-\left|s^{\prime}\right| \delta}|D|^{2}(s) \Delta^{3}(s)+\mathcal{O}\left(\delta^{2} \tau\right) \\
& =\frac{2 \delta \varepsilon}{3} \int_{0}^{\frac{t}{\varepsilon}} \mathrm{d} s \int_{-\infty}^{\infty} \mathrm{d} s^{\prime} \mathrm{e}^{-\left|s^{\prime}\right| \delta}|D|^{2}(s) \Delta^{3}(s)+\mathcal{O}\left(\delta^{2} \tau+\mathrm{e}^{-\tau \delta}+\varepsilon \tau\right) \\
& =\frac{4 \varepsilon}{3} \int_{0}^{\frac{t}{\varepsilon}} \mathrm{d} s|D|^{2}(s) \Delta^{3}(s)+\mathcal{O}\left(\delta^{2} \tau+\mathrm{e}^{-\tau \delta}+\varepsilon \tau\right) .
\end{aligned}
$$

So we end up with

$$
\begin{aligned}
\Theta_{i j}(t) & =\varepsilon^{3 \beta-1}\left\langle\Psi, \varepsilon^{2} \int_{0}^{\frac{t}{\varepsilon}} \mathrm{d} s \int_{-a(s)}^{a(s)} \mathrm{d} s^{\prime} I\left(s, s^{\prime}\right) \Psi\right\rangle \\
& =\varepsilon^{3 \beta} \int_{0}^{t / \varepsilon} \frac{4}{3}\left\langle\Psi,|D|^{2}(s) \Delta^{3}(s) \Psi\right\rangle \mathrm{d} s+o\left(\varepsilon^{3 \beta-1}\right) \\
& =\varepsilon^{3 \beta-1} \int_{0}^{t} \frac{4}{3}\left\|\left|D_{i j}\right| \Delta_{E}^{3 / 2} \mathrm{e}^{-\mathrm{i} \frac{s}{\varepsilon} H_{j}^{\varepsilon}} P_{j} \psi\right\|^{2} \mathrm{~d} s+o\left(\varepsilon^{3 \beta-1}\right) .
\end{aligned}
$$


We still need to show that the contribution of $t_{2}$ from 35 to the transitions is negligible at leading order. More precisely, we need to show that

$$
\begin{aligned}
\tilde{\Theta}_{i j}(t) & :=\left\langle\Psi, \int_{0}^{t} \mathrm{~d} r \int_{0}^{t} \mathrm{~d} r^{\prime} \mathrm{e}^{\mathrm{i} \frac{r}{\varepsilon} H_{j, \mathrm{f}}^{\varepsilon}} t_{2}^{*} \mathrm{e}^{-\mathrm{i} \frac{r}{\varepsilon} H_{i, \mathrm{f}}^{\varepsilon}} \mathrm{e}^{\mathrm{i} \frac{r^{\prime}}{\varepsilon} H_{i, \mathrm{f}}^{\varepsilon}} t_{2} \mathrm{e}^{-\mathrm{i} \frac{r^{\prime}}{\varepsilon} H_{j, \mathrm{f}}^{\varepsilon} \Psi}\right\rangle \\
& =\left\langle\Psi, \varepsilon^{2} \int_{0}^{\frac{t}{\varepsilon}} \mathrm{d} s \int_{-a(s)}^{a(s)} \mathrm{d} s^{\prime} \mathrm{e}^{\mathrm{i}\left(s+\frac{s^{\prime}}{2}\right) H_{j, \mathrm{f}}^{\varepsilon}} t_{2}^{*} \mathrm{e}^{-\mathrm{i} s^{\prime} H_{i, \mathrm{f}}^{\varepsilon}} t_{2} \mathrm{e}^{-\mathrm{i}\left(s-\frac{s^{\prime}}{2}\right) H_{j, \mathrm{f}}^{\varepsilon}} \Psi\right\rangle,
\end{aligned}
$$

is $o\left(\varepsilon^{3 \beta-1}\right)$. With the same type of arguments as in the previous proof one can now show that the main contribution to $\tilde{\Theta}_{i j}(t)$ comes from the integral

$$
\frac{8 \varepsilon^{3}}{3 \pi} \int_{0}^{\frac{t}{\varepsilon}} \mathrm{d} s\left\langle\Psi_{\ell}(s), \int_{-\tau}^{\tau} \mathrm{d} s^{\prime} \lim _{\rho \rightarrow \infty} \int_{-\rho}^{\rho} \mathrm{d} R \mathrm{e}^{-\mathrm{i} s^{\prime} R} \frac{\partial_{\ell} \Delta(s) D^{*}(s) \Delta(s)}{(R-\Delta(s)-\mathrm{i} \delta)^{2}} \mathrm{e}^{\mathrm{i} s^{\prime} \Delta(s)} \frac{\partial_{i} \Delta(s) D(s) \Delta(s)}{(R-\Delta(s)+\mathrm{i} \delta)^{2}} \Psi_{i}(s)\right\rangle
$$

which is easily seen to be $\mathcal{O}\left(\varepsilon^{2}\left(\frac{\tau}{\delta^{3}}+\frac{\tau^{2}}{\delta^{2}}\right)\right)$ after performing the $R$ integration. Here $\Psi_{\ell}(s):=\mathrm{e}^{\mathrm{i} s H_{j}^{\varepsilon}}\left(-\mathrm{i} \varepsilon \partial_{x_{\ell}}\right) \mathrm{e}^{-\mathrm{i} s H_{j}^{\varepsilon}} \Psi \in D_{0}$. This concludes to proof of Theorem 1 .

\section{Proofs of the main propositions}

Before giving the details of the proofs let us shortly comment on the relation and differences between the Propositions 1, 2 and 3. In some sense they are all "adiabatic theorems", however, of slightly different spirit. In Proposition 1 we adapt and simplify arguments from [SpTe, which in turn were motivated by Kato's proof of the adiabatic theorem of quantum mechanics for Hamiltonians slowly depending on time. The basic idea is to show that the transitions between adiabatic subspaces are small even for long times by explicitly evaluating an oscillatory integral. In Proposition 2 we use the idea of superadiabatic perturbation theory: the adiabatic subspaces are replaced by slightly tilted superadiabatic subspaces. The coupling between the superadiabatic subspaces is so small that the transitions between them can be estimated even for long times by a crude norm-estimate of the integrand. The technical reason that forces us to include the weaker statement of Proposition 1 is that it can be easily proven without energy cutoffs. This is crucial when replacing the full time-evolution by its adiabatic approximation in the computation (34) in the proof of Theorem 1 .

An essential input for adiabatic decoupling and thus for all proofs in this section is the fact that the smoothness of $H_{\mathrm{el}}(x)$ and the gap assumption imply the smoothness of the map $P_{j}: \mathbb{R}^{3 l} \rightarrow \mathcal{L}\left(\mathcal{H}_{\mathrm{el}}\right), x \mapsto P_{j}(x)$.

Lemma 4. Let $E_{j}$ be an isolated energy band and $P_{j}$ the corresponding band projection. Then $P_{j} \in C_{\mathrm{b}}^{\infty}\left(\mathbb{R}^{3 l}, \mathcal{L}\left(\mathcal{H}_{\mathrm{el}}\right)\right)$ and $E_{j} \in C_{\mathrm{b}}^{\infty}\left(\mathbb{R}^{3 l}\right)$. Moreover, for any $\alpha \in \mathbb{N}_{0}^{3 l}$ and any $n \in \mathbb{N}_{0}$ one has $\partial_{x}^{\alpha} P_{j}(x) \in \mathcal{L}\left(\mathcal{H}_{\mathrm{el}}, D\left(H_{\mathrm{el}}^{n}\right)\right)$.

Apart from $P_{j}(x)$ there will appear numerous operator-valued multiplication operators of this type and in addition also differential operators $\partial_{x}^{\alpha}$ with operator-valued coefficients. The following lemma will turn out useful when working with these kind of operators. 
Lemma 5. Let $A_{\alpha}: \mathbb{R}^{3 l} \rightarrow \mathcal{L}\left(\mathcal{H}_{\mathrm{el}}\right)$ be bounded, smooth and with bounded derivatives, i.e. $A_{\alpha} \in C_{\mathrm{b}}^{\infty}\left(\mathbb{R}^{3 l}, \mathcal{L}\left(\mathcal{H}_{\mathrm{el}}\right)\right)$. Then $A_{\alpha}=\int^{\oplus} A_{\alpha}(x) \mathrm{d} x$ defines a bounded operator on $\mathcal{H}_{\mathrm{mol}}=L^{2}\left(\mathbb{R}^{3 l} ; \mathcal{H}_{\mathrm{el}}\right)$ and we call the differential operator

$$
A^{\varepsilon}=\sum_{|\alpha|=0}^{n} A_{\alpha} \varepsilon^{\alpha} \partial_{x}^{\alpha}
$$

an admissible operator of order $n$.

(i) An admissible operator of order $n$ is bounded in $\mathcal{L}\left(D_{\mathrm{mol}}^{m}, \mathcal{H}_{\mathrm{mol}}\right)$ for $m=\lceil n / 2\rceil$ uniformly in $\varepsilon>0$.

(ii) If all coefficients $A_{\alpha}$ of an admissible operator $A^{\varepsilon}$ of order $n$ have the property that $\left[\left(H_{\mathrm{mol}}^{\varepsilon}\right)^{k}, A_{\alpha}\right]$ is an admissible operator of order $2 k-1$, then $A^{\varepsilon}$ is uniformly bounded in $\mathcal{L}\left(D_{\mathrm{mol}}^{k+m}, D_{\mathrm{mol}}^{k}\right)$ for $m=\lceil n / 2\rceil$.

As a first simple application we note the following corollary.

Corollary 3. $\partial_{x}^{\beta} P_{j}$ is uniformly bounded in $\mathcal{L}\left(D_{\text {mol }}^{n}\right)$ for all $n \in \mathbb{N}_{0}$ and $\beta \in \mathbb{N}_{0}^{3 l}$.

Proof. According to Lemma $4 . \partial_{x}^{\beta} P_{j}$ is an admissible operator of order 0 for any $\beta \in$ $\mathbb{N}_{0}^{3 l}$. Statement (ii) of Lemma 5 implies the claim of the corollary once we show that $\left[\left(H_{\mathrm{mol}}^{\varepsilon}\right)^{n}, \partial_{x}^{\beta} P_{j}\right]$ are admissible operators of order $2 n-1$ for any $n \in \mathbb{N}$. This in turn follows from direct computation and the fact that according to Lemma 4 we have $\partial_{x}^{\alpha} P_{j}(x) \in \mathcal{L}\left(\mathcal{H}_{\mathrm{el}}, D\left(H_{\mathrm{el}}^{n}\right)\right)$.

\subsection{Proof of Proposition 1}

Since $\varepsilon \partial_{x_{i}}$ has norm one in $\mathcal{L}\left(D_{\text {mol }}^{n+1}, D_{\text {mol }}^{n}\right)$, Corollary 3 implies that the commutator

$$
\left[H_{\mathrm{mol}}^{\varepsilon}, P_{j}\right]=\left[-\varepsilon^{2} \Delta_{x}, P_{j}\right]=-\varepsilon^{2}\left(\Delta_{x} P_{j}\right)-2 \varepsilon \nabla_{x} P_{j} \cdot \varepsilon \nabla_{x}=\mathcal{O}(\varepsilon)
$$

is of order $\varepsilon$ in $\mathcal{L}\left(D_{\text {mol }}^{n+1}, D_{\text {mol }}^{n}\right)$ for all $n \in \mathbb{N}_{0}$. Set $P_{j}^{\perp}(x):=1-P_{j}(x)$. Since

$$
H_{\mathrm{mol}}^{\varepsilon}-H_{j}^{\varepsilon}=P_{j} H_{\mathrm{mol}}^{\varepsilon} P_{j}^{\perp}+P_{j}^{\perp} H_{\mathrm{mol}}^{\varepsilon} P_{j}=\left(1-2 P_{j}\right)\left[H_{\mathrm{mol}}^{\varepsilon}, P_{j}\right],
$$

the self-adjointness of $\left(H_{j}^{\varepsilon}, D_{\mathrm{mol}}\right)$ for $\varepsilon$ small enough follows from Lemma 3 .

We notice that $\left\|\mathrm{e}^{-\mathrm{i} \frac{t}{\varepsilon} H_{\mathrm{mol}}^{\varepsilon}}\right\|_{\mathcal{L}\left(D_{\mathrm{mol}}\right)}=1$ and $\left\|\mathrm{e}^{-\mathrm{i} \frac{t}{\varepsilon} H_{j}^{\varepsilon}}\right\|_{\mathcal{L}\left(D\left(H_{j}^{\varepsilon}\right)\right)}=1$ for all $t \in \mathbb{R}$, when $D\left(H_{j}^{\varepsilon}\right)$ is equipped with the graph norm. Then the equivalence of the graph norms due to Lemma 3 implies that $\left\|\mathrm{e}^{-\mathrm{i} \frac{t}{\varepsilon} H_{j}^{\varepsilon}}\right\|_{\mathcal{L}\left(D_{\mathrm{mol}}\right)}$ is bounded independently of $\varepsilon$.

Now set $R_{j}(x):=P_{j}^{\perp}(x)\left(H_{\mathrm{el}}(x)-E_{j}(x)\right)^{-1} P_{j}^{\perp}(x)$ and

$$
\begin{aligned}
K_{j}(x) & :=R_{j}(x) H_{\mathrm{mol}}^{\varepsilon} P_{j}(x)+P_{j}(x) H_{\mathrm{mol}}^{\varepsilon} R_{j}(x) \\
& =R_{j}(x)\left[H_{\mathrm{mol}}^{\varepsilon}, P_{j}(x)\right] P_{j}(x)-P_{j}(x)\left[H_{\mathrm{mol}}^{\varepsilon}, P_{j}(x)\right] R_{j}(x) .
\end{aligned}
$$

Due to (46) we have that $\left[H_{\mathrm{mol}}^{\varepsilon}, P_{j}\right] K_{j},\left[E_{j}, K_{j}\right]$, and $\left[-\varepsilon^{2} \Delta_{x}, K_{j}\right]$ are of order $\varepsilon^{2}$ in $\mathcal{L}\left(D_{\text {mol }}^{n+1}, \overrightarrow{D_{\text {mol }}^{n}}\right)$. Thus it holds that

$$
\left[H_{\mathrm{mol}}^{\varepsilon}, K_{j}\right]=\left[H_{\mathrm{el}}, K_{j}\right]+\mathcal{O}\left(\varepsilon^{2}\right)=P_{j}^{\perp} H_{\mathrm{mol}}^{\varepsilon} P_{j}+P_{j} H_{\mathrm{mol}}^{\varepsilon} P_{j}^{\perp}+\mathcal{O}\left(\varepsilon^{2}\right)
$$


and therefore

$$
\begin{aligned}
& \frac{\mathrm{d}}{\mathrm{d} s}\left(\mathrm{e}^{-\mathrm{i} \frac{t-s}{\varepsilon} H_{j}^{\varepsilon}} K_{j} \mathrm{e}^{-\mathrm{i} \frac{s}{\varepsilon} H_{\mathrm{mol}}^{\varepsilon}}\right) \\
& =\frac{\mathrm{i}}{\varepsilon} \mathrm{e}^{-\mathrm{i} \frac{t-s}{\varepsilon} H_{j}^{\varepsilon}}\left(H_{j}^{\varepsilon} K_{j}-K_{j} H_{\mathrm{mol}}^{\varepsilon}\right) \mathrm{e}^{-\mathrm{i} \frac{s}{\varepsilon} H_{\mathrm{mol}}^{\varepsilon}} \\
& =\frac{\mathrm{i}}{\varepsilon} \mathrm{e}^{-\mathrm{i} \frac{t-s}{\varepsilon} H_{j}^{\varepsilon}}\left(\left(H_{\mathrm{mol}}^{\varepsilon}+\left(2 P_{j}-1\right)\left[H_{\mathrm{mol}}^{\varepsilon}, P_{j}\right]\right) K_{j}-K_{j} H_{\mathrm{mol}}^{\varepsilon}\right) \mathrm{e}^{-\mathrm{i} \frac{s}{\varepsilon} H_{\mathrm{mol}}^{\varepsilon}} \\
& =\frac{\mathrm{i}}{\varepsilon} \mathrm{e}^{-\mathrm{i} \frac{t-s}{\varepsilon} H_{j}^{\varepsilon}}\left(\left[H_{\mathrm{mol}}^{\varepsilon}, K_{j}\right]\right) \mathrm{e}^{-\mathrm{i} \frac{s}{\varepsilon} H_{\mathrm{mol}}^{\varepsilon}}+\mathcal{O}(\varepsilon) \\
& =\frac{\mathrm{i}}{\varepsilon} \mathrm{e}^{-\mathrm{i} \frac{t-s}{\varepsilon} H_{j}^{\varepsilon}}\left(P_{j}^{\perp} H_{\mathrm{mol}}^{\varepsilon} P_{j}+P_{j} H_{\mathrm{mol}}^{\varepsilon} P_{j}^{\perp}\right) \mathrm{e}^{-\mathrm{i} \frac{s}{\varepsilon} H_{\mathrm{mol}}^{\varepsilon}}+\mathcal{O}(\varepsilon)
\end{aligned}
$$

in $\mathcal{L}\left(D_{\text {mol }}^{n+1}, D_{\text {mol }}^{n}\right)$. Hence the difference in the unitary groups is

$$
\begin{aligned}
\mathrm{e}^{-\mathrm{i} \frac{t}{\varepsilon} H_{j}^{\varepsilon}}-\mathrm{e}^{-\mathrm{i} \frac{t}{\varepsilon} H_{\mathrm{mol}}^{\varepsilon}} & =\frac{\mathrm{i}}{\varepsilon} \int_{0}^{t} \mathrm{e}^{-\mathrm{i} \frac{t-s}{\varepsilon} H_{j}^{\varepsilon}}\left(H_{\mathrm{mol}}^{\varepsilon}-H_{j}^{\varepsilon}\right) \mathrm{e}^{-\mathrm{i} \frac{s}{\varepsilon} H_{\mathrm{mol}}^{\varepsilon}} \mathrm{d} s \\
& =\frac{\mathrm{i}}{\varepsilon} \int_{0}^{t} \mathrm{e}^{-\mathrm{i} \frac{t-s}{\varepsilon} H_{j}^{\varepsilon}}\left(P_{j}^{\perp} H_{\mathrm{mol}}^{\varepsilon} P_{j}+P_{j} H_{\mathrm{mol}}^{\varepsilon} P_{j}^{\perp}\right) \mathrm{e}^{-\mathrm{i} \frac{s}{\varepsilon} H_{\mathrm{mol}}^{\varepsilon}} \mathrm{d} s \\
& =\int_{0}^{t} \frac{\mathrm{d}}{\mathrm{d} s}\left(\mathrm{e}^{-\mathrm{i} \frac{t-s}{\varepsilon} H_{j}^{\varepsilon}} K_{j} \mathrm{e}^{-\mathrm{i} \frac{s}{\varepsilon} H_{\mathrm{mol}}^{\varepsilon}}\right) \mathrm{d} s+\mathcal{O}(\varepsilon|t|) \\
& =K_{j} \mathrm{e}^{-\mathrm{i} \frac{t}{\varepsilon} H_{\mathrm{mol}}^{\varepsilon}}-\mathrm{e}^{-\mathrm{i} \frac{t}{\varepsilon} H_{j}^{\varepsilon}} K_{j}+\mathcal{O}(\varepsilon|t|) .
\end{aligned}
$$

Since $K_{j}$ is of order $\varepsilon$ in $\mathcal{L}\left(D_{\text {mol }}^{n+1}, D_{\text {mol }}^{n}\right)$, we obtain 10 .

\subsection{Proof of Proposition 2}

This construction has been done in different places using different techniques. For the most general treatment of the Born-Oppenheimer approximation allowing even nuclei that are point charges we refer to the recent work of Martinez and Sordoni $\mathrm{MaSo}_{2}$ based on a twisted pseudo-differential calculus. Since the precise statements we need for treating the coupling to the field do not follow from their results, we give a more elementary proof for the case of smeared nuclei here. It is partly an adaption of the arguments used in WaTe in a different context.

For better readability we now drop the index $j$ and write $P_{0}:=P_{j}$ and $E_{*}:=E_{j}$. To have some margin to play with we use first the characteristic function $\mathbf{1}_{E+1}$ on $(-\infty, E+1]$ and recall that with $e$ denoting the infimum of the spectrum of $H_{\mathrm{mol}}^{\varepsilon}$ we have that $\mathbf{1}_{E+1}\left(H_{\mathrm{mol}}^{\varepsilon}\right)=\mathbf{1}_{[e, E+1]}\left(H_{\mathrm{mol}}^{\varepsilon}\right)$.

Starting from the orthogonal projection $P_{0}$ we want to construct a self-adjoint operator $P^{\varepsilon} \in \mathcal{L}\left(\mathcal{H}_{\text {mol }}\right)$ with

$$
P^{\varepsilon} P^{\varepsilon}=P^{\varepsilon} \quad \text { and } \quad\left[H_{\mathrm{mol}}^{\varepsilon}, P^{\varepsilon}\right] \mathbf{1}_{E+1}\left(H_{\mathrm{mol}}^{\varepsilon}\right)=\mathcal{O}\left(\varepsilon^{3}\right)
$$

The first statement just means that $P^{\varepsilon}$ is a projection. The basic idea for constructing $P^{\varepsilon}$ is to determine first the coefficients in an asymptotic expansion

$$
P^{\varepsilon}=P_{0}+\varepsilon P_{1}+\varepsilon^{2} P_{2}+\mathcal{O}\left(\varepsilon^{3}\right),
$$


where we recall that according to 46 the commutator $\left[H_{\mathrm{mol}}^{\varepsilon}, P_{0}\right]$ with the choice $P_{0}=$ $P_{j}$ is of order $\varepsilon$ as an operator in $\mathcal{L}\left(D_{\text {mol }}^{n+1}, D_{\text {mol }}^{n}\right)$. As shown in many instances, the requirements that $P^{(2)}:=P_{0}+\varepsilon P_{1}+\varepsilon^{2} P_{2}$ satisfies

$$
P^{(2)} P^{(2)}-P^{(2)}=\mathcal{O}\left(\varepsilon^{3}\right) \quad \text { and } \quad\left[P^{(2)}, H_{\mathrm{mol}}^{\varepsilon}\right]=\mathcal{O}\left(\varepsilon^{3}\right)
$$

fix $P^{(2)}$ uniquely modulo terms of order $\varepsilon^{3}$. We will not repeat the construction here, but only give the result: Let

$$
\left[P_{0}\right]:=\frac{1}{\varepsilon}\left[H_{\mathrm{mol}}^{\varepsilon}, P_{0}\right],
$$

then $\left[P_{0}\right]$ is, according to 46 , a uniformly bounded operator in $\mathcal{L}\left(D_{\text {mol }}^{n+1}, D_{\text {mol }}^{n}\right)$. We put

$$
S_{1}:=P_{0}\left[P_{0}\right] R
$$

with the reduced resolvent $R(x)=P_{0}(x)^{\perp}\left(H_{\mathrm{el}}(x)-E_{*}(x)\right)^{-1} P_{0}(x)^{\perp}$. Since $\partial_{x}^{\alpha} H_{\mathrm{el}}(x)$ and thus also $\partial_{x}^{\alpha} R(x)$ are bounded operator on $\mathcal{H}_{\text {el }}$ for any $\alpha \in \mathbb{N}^{3 l}, S_{1}$ is an admissible operator of order one in the sense of Lemma 5. This is where smearing out the nuclear charge distribution is essential. By the same reasoning as in (47) this choice makes

$$
\left[P_{0}\right]+\left[H_{\mathrm{el}}, S_{1}+S_{1}^{*}\right]=\mathcal{O}(\varepsilon) .
$$

Now let

$$
P_{1}:=S_{1}+S_{1}^{*} \quad \text { and } \quad P^{(1)}:=P_{0}+\varepsilon P_{1},
$$

then

$$
\begin{aligned}
P^{(1)} P^{(1)}-P^{(1)} & =\varepsilon\left(P_{0} P_{1}+P_{1} P_{0}-P_{1}\right)+\varepsilon^{2} P_{1} P_{1} \\
& =\varepsilon\left(S_{1}+S_{1}^{*}-P_{1}\right)+\varepsilon^{2}\left(S_{1} S_{1}^{*}+S_{1}^{*} S_{1}\right)=\varepsilon^{2}\left(S_{1} S_{1}^{*}+S_{1}^{*} S_{1}\right)
\end{aligned}
$$

and

$$
\left[H_{\mathrm{mol}}^{\varepsilon}, P^{(1)}\right]=\varepsilon\left[P_{0}\right]+\varepsilon\left[H_{\mathrm{el}}, S_{1}+S_{1}^{*}\right]+\varepsilon^{2}\left[-\varepsilon \Delta, P_{1}\right]=\mathcal{O}\left(\varepsilon^{2}\right)
$$

in $\mathcal{L}\left(D_{\text {mol }}^{n+1}, D_{\text {mol }}^{n}\right)$. Now we simply iterate this construction: first we modify $P^{(1)}$ in order to make it a projection to higher order by putting

$$
\tilde{P}^{(1)}:=P^{(1)}+\varepsilon^{2}\left(S_{1}^{*} S_{1}-S_{1} S_{1}^{*}\right) .
$$

This gives

$$
\tilde{P}^{(1)} \tilde{P}^{(1)}-\tilde{P}^{(1)}=P^{(1)} P^{(1)}-P^{(1)}-2 \varepsilon^{2} S_{1} S_{1}^{*}-\varepsilon^{2}\left(S_{1}^{*} S_{1}-S_{1} S_{1}^{*}\right)+\mathcal{O}\left(\varepsilon^{3}\right)=\mathcal{O}\left(\varepsilon^{3}\right) .
$$

Then we put

$$
\left[\tilde{P}^{(1)}\right]:=\frac{1}{\varepsilon^{2}}\left[H_{\mathrm{mol}}^{\varepsilon}, \tilde{P}^{(1)}\right]
$$

and

$$
S_{2}=P_{0}\left[\tilde{P}^{(1)}\right] R,
$$

which makes

$$
\left[\tilde{P}^{(1)}\right]+\left[H_{\mathrm{el}}, S_{2}+S_{2}^{*}\right]=\mathcal{O}(\varepsilon) .
$$


Defining

$$
P_{2}=S_{2}+S_{2}^{*}+S_{1}^{*} S_{1}-S_{1} S_{1}^{*}
$$

we find that

$$
\left[H_{\mathrm{mol}}^{\varepsilon}, P^{(2)}\right]=\varepsilon^{2}\left[\tilde{P}^{(1)}\right]+\varepsilon^{2}\left[H_{\mathrm{el}}, S_{2}+S_{2}^{*}\right]+\varepsilon^{3}\left[-\varepsilon \Delta, S_{2}+S_{2}^{*}\right]=: \varepsilon^{3} R_{1}^{\varepsilon},
$$

and still

$$
P^{(2)} P^{(2)}-P^{(2)}=\tilde{P}^{(1)} \tilde{P}^{(1)}+\varepsilon^{2}\left(S_{2}+S_{2}^{*}\right)-\left(\tilde{P}^{(1)}+\varepsilon^{2}\left(S_{2}+S_{2}^{*}\right)\right)+\mathcal{O}\left(\varepsilon^{3}\right)=: \varepsilon^{3} R_{2}^{\varepsilon} .
$$

Note that $R_{1}^{\varepsilon}$ is an admissible operator of order three and $R_{2}^{\varepsilon}$ is an admissible operator of order four. The following lemma shows that all the operators appearing in the construction can be bounded by appropriate powers of $H_{\mathrm{mol}}^{\varepsilon}$.

Lemma 6. The operators $P_{0}, P_{1}, P_{2}, R_{1}^{\varepsilon}$ and $R_{2}^{\varepsilon}$ are admissible operators of order 0 , $1,2,3$, and 4 respectively. Their coefficients have commutators with $\left(H_{\mathrm{mol}}^{\varepsilon}\right)^{k}$ that are admissible operators of order $2 k-1$ for any $k \in \mathbb{N}$. Thus they are uniformly bounded operators from $D_{\mathrm{mol}}^{n+m}$ to $D_{\mathrm{mol}}^{n}$ for any $n \in \mathbb{N}_{0}$ and $m=0,1,2,3$, and 4 respectively.

In order to make sense of (48) as a bounded operator in $\mathcal{L}\left(\mathcal{H}_{\mathrm{mol}}\right)$ and to get uniform bounds on $\left[H_{\mathrm{mol}}^{\varepsilon}, P^{\varepsilon}\right]$ we thus need to cut off large energies. To do so we fix $E<\infty$ and choose $\chi_{E} \in C_{0}^{\infty}(\mathbb{R},[0,1])$ such that $\left.\chi_{E}\right|_{[e-1, E+1]}=1$ and $\operatorname{supp} \chi_{E} \subset(e-2, E+2)$. Then we define

$$
\tilde{P}^{\varepsilon}:=\varepsilon P_{1}+\varepsilon^{2} P_{2}
$$

and

$$
P_{\chi_{E}}^{\varepsilon}:=P_{0}+\varepsilon P_{1}+\varepsilon^{2} P_{2}-\left(1-\chi_{E}\left(H_{\mathrm{mol}}^{\varepsilon}\right)\right) \tilde{P}^{\varepsilon}\left(1-\chi_{E}\left(H_{\mathrm{mol}}^{\varepsilon}\right)\right),
$$

i.e. we cut off the corrections to $P_{0}$ at high energies. To see that $P_{\chi_{E}}^{\varepsilon}$ is indeed a bounded operator in $\mathcal{L}\left(D_{\text {mol }}^{n}\right)$ for any $n \in \mathbb{N}_{0}$, note that

$$
P_{\chi_{E}}^{\varepsilon}=P_{0}+\tilde{P}^{\varepsilon} \chi_{E}\left(H_{\mathrm{mol}}^{\varepsilon}\right)+\chi_{E}\left(H_{\mathrm{mol}}^{\varepsilon}\right) \tilde{P}^{\varepsilon}\left(1-\chi_{E}\left(H_{\mathrm{mol}}^{\varepsilon}\right)\right)
$$

and that $P_{0}$ and $\tilde{P}^{\varepsilon} \chi_{E}\left(H_{\text {mol }}^{\varepsilon}\right)$ are bounded independently of $\varepsilon$ in $\mathcal{L}\left(D_{\text {mol }}^{n}\right)$ by Lemma 6 and the fact that $\chi_{E}\left(H_{\text {mol }}^{\varepsilon}\right) \in \mathcal{L}\left(\mathcal{H}_{\text {mol }}, D_{\text {mol }}^{n}\right)$ with norm bounded independently of $\varepsilon$. In particular we have also

$$
\left\|P_{\chi_{E}}^{\varepsilon}-P_{0}\right\|_{\mathcal{L}\left(D_{\text {mol }}^{n}\right)}=\mathcal{O}(\varepsilon)
$$

We first proof that the operator $P_{\chi_{E}}^{\varepsilon}$ has all the properties claimed in the proposition modulo the fact that it is not a projection. In a second step we turn it into a projection without loosing the desired properties.

Now by Lemma 6 it follows that

$$
\left[H_{\mathrm{mol}}^{\varepsilon}, P_{\chi_{E}}^{\varepsilon}\right]=\left[H_{\mathrm{mol}}^{\varepsilon}, P_{0}\right]+\mathcal{O}(\varepsilon)=\mathcal{O}(\varepsilon)
$$

as a bounded operator from $D_{\mathrm{mol}}^{n+1}$ to $D_{\mathrm{mol}}^{n}$. With $\chi_{E}\left(H_{\mathrm{mol}}^{\varepsilon}\right) \mathbf{1}_{E+1}\left(H_{\mathrm{mol}}^{\varepsilon}\right)=\mathbf{1}_{E+1}\left(H_{\mathrm{mol}}^{\varepsilon}\right)$ this implies

$$
\begin{aligned}
{\left[H_{\mathrm{mol}}^{\varepsilon}, P_{\chi_{E}}^{\varepsilon}\right] \mathbf{1}_{E+1}\left(H_{\mathrm{mol}}^{\varepsilon}\right) } & =\left[H_{\mathrm{mol}}^{\varepsilon}, P_{0}+\varepsilon P_{1}+\varepsilon^{2} P_{2}\right] \mathbf{1}_{E+1}\left(H_{\mathrm{mol}}^{\varepsilon}\right) \\
& =\varepsilon^{3} R_{1}^{\varepsilon} \mathbf{1}_{E+1}\left(H_{\mathrm{mol}}^{\varepsilon}\right)=\mathcal{O}\left(\varepsilon^{3}\right)
\end{aligned}
$$


as an operator from $\mathcal{H}_{\text {mol }}$ to $D_{\text {mol }}^{n}$. Note that, by taking adjoints, this implies that

$$
\left\|\mathbf{1}_{E+1}\left(H_{\mathrm{mol}}^{\varepsilon}\right)\left[H_{\mathrm{mol}}^{\varepsilon}, P_{\chi_{E}}^{\varepsilon}\right]\right\|_{\mathcal{L}\left(\mathcal{H}_{\mathrm{mol}}\right)}=\mathcal{O}\left(\varepsilon^{3}\right)
$$

and with $\left\|\mathbf{1}_{E+1}\left(H_{\text {mol }}^{\varepsilon}\right)\right\|_{\mathcal{L}\left(\mathcal{H}_{\mathrm{mol}}, D_{\text {mol }}^{n}\right)}=\mathcal{O}(1)$ also

$$
\left\|\mathbf{1}_{E+1}\left(H_{\mathrm{mol}}^{\varepsilon}\right)\left[H_{\mathrm{mol}}^{\varepsilon}, P_{\chi_{E}}^{\varepsilon}\right]\right\|_{\mathcal{L}\left(\mathcal{H}_{\mathrm{mol}}, D_{\mathrm{mol}}^{n}\right)}=\mathcal{O}\left(\varepsilon^{3}\right) .
$$

For later use we also show that this implies the smallness of the commutator of $P_{\chi_{E}}^{\varepsilon}$ with a smooth energy cutoff $\tilde{\chi}$ supported in $\left(e-\frac{3}{4}, E+\frac{3}{4}\right)$

$$
\left\|\left[\tilde{\chi}\left(H_{\mathrm{mol}}^{\varepsilon}\right), P_{\chi_{E}}^{\varepsilon}\right]\right\|_{\mathcal{L}\left(\mathcal{H}_{\mathrm{mol}}, D_{\mathrm{mol}}^{n}\right)}=\mathcal{O}\left(\varepsilon^{3}\right)
$$

Since the argument will be used several times in the remainder of the paper, we formulate it as a lemma.

Lemma 7. Let $I \subset \mathbb{R}$ be a compact interval, $\tilde{I} \subset I$ another interval with different endpoints and $\tilde{\chi} \in C_{0}^{\infty}(\mathbb{R})$ with supp $\tilde{\chi} \subset \tilde{I}$. Then for any $n \in \mathbb{N}_{0}$ there exists $C<\infty$ depending only on $n$ and $\tilde{\chi}$ with the following property: Let $(H, D(H))$ be self-adjoint and $A \in \mathcal{L}(\mathcal{H})$ be bounded and self-adjoint. Then

$$
\left\|[H, A] \mathbf{1}_{I}(H)\right\|_{\mathcal{L}\left(\mathcal{H}, D\left(H^{n}\right)\right)} \leq \delta
$$

implies that

$$
\|[\tilde{\chi}(H), A]\|_{\mathcal{L}\left(\mathcal{H}, D\left(H^{n}\right)\right)} \leq C \delta .
$$

Now we need to turn the "almost projection" $P_{\chi_{E}}^{\varepsilon}$ into a true projection. Since we will use this trick as well several times, we formulate it again as a lemma.

Lemma 8. There are constants $C_{n}<\infty, n \in \mathbb{N}$, such that the following holds:

Let $(H, D(H))$ be a self-adjoint operator and let $D^{n}:=D\left(H^{n}\right)$ be equipped with the norm

$$
\|\psi\|_{D^{n}}:=\sum_{i=0}^{n}\left\|H^{i} \psi\right\| .
$$

For some $N \in \mathbb{N}$ let $\tilde{Q}$ be an operator that is bounded in $\mathcal{L}\left(D^{n}\right)$ for all $0 \leq n \leq N$ and self-adjoint in $\mathcal{L}(\mathcal{H})$ with the following properties:

$$
\|\tilde{Q} \tilde{Q}-\tilde{Q}\|_{\mathcal{L}\left(D^{n}\right)} \leq \delta
$$

for all $0 \leq n \leq N$ and some $\delta<\frac{1}{4}$ and

$$
\|[H, \tilde{Q}]\|_{\mathcal{L}\left(D^{n}, D^{n-1}\right)} \leq \delta_{n}
$$

for all $1 \leq n \leq N$ and some $\delta_{n}<\frac{1}{2} \frac{1}{2^{2 n}}$.

Then there is an orthogonal projection $Q \in \mathcal{L}(\mathcal{H})$ with $\|Q\|_{\mathcal{L}\left(D^{n}\right)} \leq 4^{n+1}$ that satisfies

$$
\|Q-\tilde{Q}\|_{\mathcal{L}(\mathcal{H})} \leq \delta \quad \text { and } \quad\|Q-\tilde{Q}\|_{\mathcal{L}\left(D^{n}\right)} \leq C_{n} \delta
$$


and

$$
\|[H, Q]\|_{\mathcal{L}\left(D^{n}, D^{n-1}\right)} \leq C_{n} \delta_{n}
$$

for all $n \leq N$.

Moreover, there is a constant $C_{E}$ depending only on $E \in \mathbb{R}$ such that we have the following implications:

$$
\left\|[H, \tilde{Q}] \mathbf{1}_{E+1}(H)\right\|_{\mathcal{L}\left(\mathcal{H}, D^{n}\right)} \leq \beta_{1}
$$

for all $n \leq N$ implies

$$
\left\|[H, Q] \mathbf{1}_{E+\frac{1}{2}}(H)\right\|_{\mathcal{L}\left(\mathcal{H}, D^{n}\right)} \leq C_{E} C_{n} \beta_{1}
$$

for all $n \leq N$, and

$$
\left\|(\tilde{Q} \tilde{Q}-\tilde{Q}) \mathbf{1}_{E+\frac{1}{2}}(H)\right\|_{\mathcal{L}\left(\mathcal{H}, D^{n}\right)} \leq \beta_{2}
$$

for all $n \leq N$ implies

$$
\left\|(Q-\tilde{Q}) \mathbf{1}_{E+\frac{1}{2}}(H)\right\|_{\mathcal{L}\left(\mathcal{H}, D^{n}\right)} \leq C_{E} C_{n} \beta_{2}
$$

for all $n \leq N$.

We can now apply Lemma 8 to the almost projection $P_{\chi_{E}}^{\varepsilon}$ almost commuting with $H_{\mathrm{mol}}^{\varepsilon}$, where now $\delta$ and $\delta_{n}$ are of order $\varepsilon$ and $\beta_{1}$ of order $\varepsilon^{3}$. To be able to use also the last implication of Lemma 8 with $\beta_{2}$ of order $\varepsilon^{3}$, we still need to show (54). To this end observe that we have by construction that

$$
\left\|\left(P^{(2)} P^{(2)}-P^{(2)}\right) \mathbf{1}_{E+\frac{1}{2}}\left(H_{\mathrm{mol}}^{\varepsilon}\right)\right\|_{\mathcal{L}\left(\mathcal{H}_{\mathrm{mol}}, D_{\mathrm{mol}}^{n}\right)}=\mathcal{O}\left(\varepsilon^{3}\right) .
$$

Hence for $\tilde{\chi} \in C_{0}^{\infty}(\mathbb{R})$ with $\tilde{\chi} \mathbf{1}_{E+\frac{1}{2}}=\mathbf{1}_{E+\frac{1}{2}}$ and $\operatorname{supp} \tilde{\chi} \subset\left(e-\frac{3}{4}, E+\frac{3}{4}\right)$ we have

$$
\begin{aligned}
\left(\left(P_{\chi_{E}}^{\varepsilon}\right)^{2}-P_{\chi_{E}}^{\varepsilon}\right) \mathbf{1}_{E+\frac{1}{2}}\left(H_{\mathrm{mol}}^{\varepsilon}\right) & =\left(\left(P_{\chi_{E}}^{\varepsilon}\right)^{2}-P_{\chi_{E}}^{\varepsilon}\right) \tilde{\chi}\left(H_{\mathrm{mol}}^{\varepsilon} \mathbf{1}_{E+\frac{1}{2}}\left(H_{\mathrm{mol}}^{\varepsilon}\right)\right. \\
& \stackrel{(49)}{=} \tilde{\chi}\left(H_{\mathrm{mol}}^{\varepsilon}\right)\left(\left(P_{\chi_{E}}^{\varepsilon}\right)^{2}-P_{\chi_{E}}^{\varepsilon}\right) \mathbf{1}_{E+\frac{1}{2}}\left(H_{\mathrm{mol}}^{\varepsilon}\right)+\mathcal{O}\left(\varepsilon^{3}\right) \\
& =\tilde{\chi}\left(H_{\mathrm{mol}}^{\varepsilon}\right)\left(\left(P^{(2)}\right)^{2}-P^{(2)}\right) \mathbf{1}_{E+\frac{1}{2}}\left(H_{\mathrm{mol}}^{\varepsilon}\right)+\mathcal{O}\left(\varepsilon^{3}\right) \\
& =\mathcal{O}\left(\varepsilon^{3}\right) .
\end{aligned}
$$

Thus we can use Lemma 8 to turn $P_{\chi_{E}}^{\varepsilon}$ into an orthogonal projection $P^{\varepsilon}$ with the desired properties.

Next we show that

$$
P_{j}^{(1)} P_{i}^{(1)}=\mathcal{O}\left(\varepsilon^{2}\right) \quad \text { for } i \neq j .
$$

To enhance readability we denote $P_{j}^{(1)}=P^{(1)}$ and $P_{i}^{(1)}=Q^{(1)}$ etc., i.e. we distinguish the different electronic levels by the letters $P$ and $Q$ instead of the indices $j$ and $i$. Then with $Q_{0} P_{0}=0$

$$
Q^{(1)} P^{(1)}=\left(Q_{0}+\varepsilon Q_{1}\right)\left(P_{0}+\varepsilon P_{1}\right)=\varepsilon\left(Q_{1} P_{0}+Q_{0} P_{1}\right)+\mathcal{O}\left(\varepsilon^{2}\right) .
$$


Denoting the $S_{1}$-operator associated to $Q^{(1)}$ by $R_{1}$ we find

$$
\begin{aligned}
Q_{1} P_{0}+Q_{0} P_{1} & =R_{1} P_{0}+Q_{0} S_{1}^{*} \\
& =Q_{0}\left[Q_{0}\right] R\left(E_{Q}\right) P_{0}-Q_{0} R\left(E_{P}\right)\left[P_{0}\right] P_{0} \\
& =\left(E_{P}-E_{Q}\right)^{-1}\left(Q_{0}\left[Q_{0}\right] P_{0}+Q_{0}\left[P_{0}\right] P_{0}\right)=0 .
\end{aligned}
$$

This implies that

$$
\begin{aligned}
P^{\varepsilon} Q^{\varepsilon} \mathbf{1}_{E+\frac{1}{2}}\left(H_{\mathrm{mol}}^{\varepsilon}\right) & =P^{\varepsilon} Q^{\varepsilon} \tilde{\chi}\left(H_{\mathrm{mol}}^{\varepsilon}\right) \mathbf{1}_{E+\frac{1}{2}}\left(H_{\mathrm{mol}}^{\varepsilon}\right) \\
& =\tilde{\chi}\left(H_{\mathrm{mol}}^{\varepsilon}\right) P^{\varepsilon} Q^{\varepsilon} \mathbf{1}_{E+\frac{1}{2}}\left(H_{\mathrm{mol}}^{\varepsilon}\right)+\mathcal{O}\left(\varepsilon^{3}\right) \\
& =\tilde{\chi}\left(H_{\mathrm{mol}}^{\varepsilon}\right) P^{(1)} Q^{(1)} \mathbf{1}_{E+\frac{1}{2}}\left(H_{\mathrm{mol}}^{\varepsilon}\right)+\mathcal{O}\left(\varepsilon^{2}\right) \\
& =\mathcal{O}\left(\varepsilon^{2}\right)
\end{aligned}
$$

in $\mathcal{L}\left(\mathcal{H}_{\mathrm{mol}}, D_{\mathrm{mol}}^{n}\right)$.

\subsection{Proof of Propositions 3 \& 4}

We first recall the perturbative form of $H^{\varepsilon}$ from (8):

$$
H^{\varepsilon}=: H_{0}^{\varepsilon}+\varepsilon^{\frac{3}{2} \beta} H_{1}^{\varepsilon}+\varepsilon^{\frac{3}{2} \beta+1} H_{2}^{\varepsilon} .
$$

The operators $H_{1}^{\varepsilon}$ and $H_{2}^{\varepsilon}$ satisfy

$$
\left\|H_{i}^{\varepsilon}\right\|_{\mathcal{L}\left(D\left(H_{0}^{\varepsilon}\right), \mathcal{H}\right)} \leq C_{i}
$$

with constants $C_{i}$ independent of $\varepsilon$. Hence Lemma 3 yields that for $\varepsilon$ small enough $H^{\varepsilon}$ is self-adjoint on $D\left(H_{0}^{\varepsilon}\right)$ and the graph norms induced by $H_{0}^{\varepsilon}$ and $H^{\varepsilon}$ are uniformly equivalent.

We write $P_{\text {vac }}^{\varepsilon}:=P_{j \text {,vac }}^{\varepsilon}$ and $P_{\text {vac }}:=P_{j} \otimes Q_{0}$, where $Q_{0}$ is the projection onto the vacuum state in $\mathscr{F}$. As before we first construct an almost projection $\tilde{P}_{\text {vac }}^{\varepsilon}$ with the desired properties and then apply Lemma 8. Since the first correction to $H_{0}^{\varepsilon}$ is of order $\varepsilon^{\frac{3}{2} \beta}$, it is natural to make the ansatz

$$
\tilde{P}_{\text {vac }}^{\varepsilon}:=P^{\varepsilon} \otimes Q_{0}+\varepsilon^{\frac{3}{2} \beta} P_{\frac{3}{2} \beta},
$$

where we assume that $P^{\varepsilon}$ is constructed as in Proposition 2 but with energy cut off at $E+1$. Computing the commutator with $H^{\varepsilon}$, we find that

$$
\begin{array}{ll}
{\left[\tilde{P}_{\mathrm{vac}}^{\varepsilon}, H^{\varepsilon}\right] \mathbf{1}_{E+1}\left(H_{0}^{\varepsilon}\right)=} \\
\stackrel{56]}{=} \quad\left[P^{\varepsilon} \otimes Q_{0}+\varepsilon^{\frac{3}{2} \beta} P_{\frac{3}{2} \beta}, H_{0}^{\varepsilon}+\varepsilon^{\frac{3}{2} \beta} H_{1}^{\varepsilon}\right] \mathbf{1}_{E+1}\left(H_{0}^{\varepsilon}\right)+\mathcal{O}\left(\varepsilon^{\frac{3}{2} \beta+1}\right) \\
=\quad\left[P^{\varepsilon} \otimes Q_{0}, H_{0}^{\varepsilon}\right] \mathbf{1}_{E+1}\left(H_{0}^{\varepsilon}\right)+\varepsilon^{3 \beta}\left[P_{\frac{3}{2} \beta}, H_{1}^{\varepsilon}\right] \mathbf{1}_{E+1}\left(H_{0}^{\varepsilon}\right) \\
& +\varepsilon^{\frac{3}{2} \beta}\left(\left[P^{\varepsilon} \otimes Q_{0}, H_{1}^{\varepsilon}\right]+\left[P_{\frac{3}{2} \beta}, H_{0}^{\varepsilon}\right]\right) \mathbf{1}_{E+1}\left(H_{0}^{\varepsilon}\right)+\mathcal{O}\left(\varepsilon^{\frac{3}{2} \beta+1}\right) \\
&
\end{array}
$$


in $\mathcal{L}(\mathcal{H})$. In this computation we made the following assumptions, which are clear on a formal level but need to be proved later on:

$$
\begin{gathered}
{\left[\tilde{P}_{\mathrm{vac}}^{\varepsilon}, H_{2}^{\varepsilon}\right] \mathbf{1}_{E+1}\left(H_{0}^{\varepsilon}\right)=\mathcal{O}(1)} \\
\varepsilon^{3 \beta}\left[P_{\frac{3}{2} \beta}, H_{1}^{\varepsilon}\right] \mathbf{1}_{E+1}\left(H_{0}^{\varepsilon}\right)=\mathcal{O}\left(\varepsilon^{\frac{3}{2} \beta+1}\right) \\
{\left[P^{\varepsilon} \otimes Q_{0}, H_{0}^{\varepsilon}\right] \mathbf{1}_{E+1}\left(H_{0}^{\varepsilon}\right)=\mathcal{O}\left(\varepsilon^{\frac{3}{2} \beta+1}\right)} \\
{\left[\left(P^{\varepsilon}-P_{j}\right) \otimes Q_{0}, H_{1}^{\varepsilon}\right] \mathbf{1}_{E+1}\left(H_{0}^{\varepsilon}\right)=\mathcal{O}(\varepsilon),}
\end{gathered}
$$

all in $\mathcal{L}(\mathcal{H})$. Whether 56 and (57) are satisfied depends on $P_{\frac{3}{2} \beta}$, which we now construct by the requirement that the commutator is of order $\varepsilon^{\frac{3}{2} \beta} \delta^{\frac{1}{2}}$, i.e. that

$$
\left(\left[P_{\mathrm{vac}}, H_{1}^{\varepsilon}\right]+\left[P_{\frac{3}{2} \beta}, H_{0}^{\varepsilon}\right]\right) \mathbf{1}_{E+1}\left(H_{0}^{\varepsilon}\right)=\mathcal{O}\left(\delta^{\frac{1}{2}}\right) .
$$

Dropping the energy cutoff for a moment this translates to

$$
\left[P_{\frac{3}{2} \beta}, H_{0}^{\varepsilon}\right]=-\left[P_{\text {vac }}, H_{1}^{\varepsilon}\right]+\mathcal{O}\left(\delta^{\frac{1}{2}}\right) .
$$

To solve this equation for $P_{\frac{3}{2} \beta}$ we cannot proceed as in adiabatic theory with spectral gap, since the reduced resolvent $\left(H_{\mathrm{el}}+H_{\mathrm{f}}-E_{j}\right)^{-1}\left(1-P_{\mathrm{vac}}\right)$ is not bounded without spectral gap. Therefore we proceed as in $\left[\mathrm{Teu}_{1}\right.$ and shift the resolvent into the complex plane by a small amount $\delta$,

$$
\begin{aligned}
P_{\frac{3}{2} \beta}^{\delta} & :=-\left(H_{\mathrm{f}}+H_{\mathrm{el}}-E_{j}+\mathrm{i} \delta\right)^{-1} H_{1}^{\varepsilon} P_{\mathrm{vac}}-P_{\mathrm{vac}} H_{1}^{\varepsilon}\left(H_{\mathrm{f}}+H_{\mathrm{el}}-E_{j}-\mathrm{i} \delta\right)^{-1} \\
& =: T_{\delta}+T_{\delta}^{*} .
\end{aligned}
$$

Note that $P_{\frac{3}{2}}^{\delta=0} \beta$ is exactly the first order correction one would obtain by formally applying standard perturbation theory to the electronic eigenprojection $P_{j}(x) \otimes Q_{0}$. With this definition we find that

$$
\begin{aligned}
& {\left[P_{\frac{3}{2} \beta}^{\delta}, H_{\mathrm{f}}+H_{\mathrm{el}}\right]} \\
& =H_{1}^{\varepsilon} P_{\mathrm{vac}}-P_{\mathrm{vac}} H_{1}^{\varepsilon}+\left(E_{j}-\mathrm{i} \delta\right)\left(H_{\mathrm{f}}+H_{\mathrm{el}}-E_{j}+\mathrm{i} \delta\right)^{-1} H_{1}^{\varepsilon} P_{\mathrm{vac}} \\
& -P_{\mathrm{vac}} H_{1}^{\varepsilon}\left(H_{\mathrm{f}}+H_{\mathrm{el}}-E_{j}-\mathrm{i} \delta\right)^{-1}\left(E_{j}+\mathrm{i} \delta\right) \\
& -\left(H_{\mathrm{f}}+H_{\mathrm{el}}-E_{j}+\mathrm{i} \delta\right)^{-1} H_{1}^{\varepsilon} P_{\mathrm{vac}} E_{j}+E_{j} P_{\mathrm{vac}} H_{1}^{\varepsilon}\left(H_{\mathrm{f}}+H_{\mathrm{el}}-E_{j}-\mathrm{i} \delta\right)^{-1} \\
& =-\left[P_{\mathrm{vac}}, H_{1}^{\varepsilon}\right]+\mathrm{i} \delta\left(T_{\delta}+T_{\delta}^{*}\right) .
\end{aligned}
$$

We will show that

$$
\mathrm{i} \delta\left(T_{\delta}+T_{\delta}^{*}\right)=\mathcal{O}\left(\delta^{1 / 2}\right) \quad \text { and } \quad\left[T_{\delta}, \varepsilon \nabla_{x}\right]=\mathcal{O}\left(\frac{\varepsilon}{\delta^{3 / 2}}\right)
$$

which indeed gives us 60 for $\delta \geq \varepsilon^{\frac{1}{2}}$. Note for the following that $P_{\frac{3}{2} \beta}^{\delta}$ is again a fibered operator,

$$
T_{\delta}(x)=-\left(H_{\mathrm{f}}(x)+H_{\mathrm{el}}-E_{j}(x)+\mathrm{i} \delta\right)^{-1} H_{1}^{\varepsilon} P_{\mathrm{vac}}(x) .
$$

This is important, since we will need to commute $P_{\frac{3}{2} \beta}^{\delta}$ through $H_{\text {mol }}^{\varepsilon}$ and thus to compute derivatives of $P_{\frac{3}{2} \beta}^{\delta}(x)$ with respect to $x$. 
Lemma 9. For $\delta>0$ small enough we have that $\mathbb{R}^{3 l} \rightarrow \mathcal{L}(\mathcal{H}), x \mapsto T_{\delta}(x)$ is smooth and there is a constant $C<\infty$ not depending on $\delta$ or $\varepsilon$ such that

$$
\begin{gathered}
\left\|T_{\delta}\right\| \leq \frac{C}{\sqrt{\delta}}, \\
\left\|\left(H_{\mathrm{f}}+H_{\mathrm{el}}\right) T_{\delta}\right\| \leq \frac{C}{\sqrt{\delta}}
\end{gathered}
$$

and for $|\alpha| \in \mathbb{N}_{0}^{3 l}$

$$
\left\|\partial_{x}^{\alpha} T_{\delta}\right\| \leq C\left(\frac{1}{\delta}\right)^{|\alpha|+\frac{1}{2}} \quad \text { and } \quad\left\|\left(H_{\mathrm{f}}+H_{\mathrm{el}}\right) \partial_{x}^{\alpha} T_{\delta}\right\| \leq C\left(\frac{1}{\delta}\right)^{|\alpha|+\frac{1}{2}} .
$$

Proof. Let $\left(\varphi_{1}(x), \ldots, \varphi_{s}(x)\right)$ be an orthonormal basis of $\operatorname{Ran} P_{j}(x)$ and write $\Psi \in$ $\operatorname{Ran} P_{j} \otimes Q_{0}$ as

$$
\Psi(x, y)=\sum_{m=1}^{s} \psi_{m}(x) \varphi_{m}(x, y)
$$

Then

$$
H_{1}^{\varepsilon} P_{\mathrm{vac}} \Psi=\mathrm{i} \sum_{i=1}^{r} A\left(\varepsilon^{\beta} y_{i}\right) \cdot \nabla_{y_{i}} \Psi=\mathrm{i} \sum_{m=1}^{s} \sum_{i=1}^{r} \sum_{\lambda=1}^{2} \psi_{m}(x) \frac{e_{\lambda}(k)}{\sqrt{2|k|}} \hat{\rho}(k) \mathrm{e}^{-\mathrm{i} \varepsilon^{\beta} k \cdot y_{i}} \nabla_{y_{i}} \varphi_{m}(x, y) .
$$

Since the sum is finite, it suffices to estimate the resolvent acting on each summand. We split

$$
1=\mathbf{1}_{[e, \infty)}\left(H_{\mathrm{el}}\right)=\mathbf{1}_{\left[e, E_{*}\right]}\left(H_{\mathrm{el}}\right)+\mathbf{1}_{\left(E_{*}, \infty\right)}\left(H_{\mathrm{el}}\right)=: P_{\leq}+P_{>}
$$

and observe that on the range of $P_{>}$the resolvent is indeed uniformly bounded also for $\delta=0$ because of the gap condition. So it remains to look at the resolvent acting on the range of

$$
P_{\leq}:=\sum_{\ell=1}^{j} P_{\ell}
$$

Using $H_{\mathrm{el}} P_{\ell}=E_{\ell} P_{\ell}$ we get that

$$
\begin{aligned}
& \left\|\left(|k|+H_{\mathrm{el}}(x)\right)\left(|k|+H_{\mathrm{el}}(x)-E_{j}(x)+\mathrm{i} \delta\right)^{-1} \frac{\hat{\rho}(k)}{\sqrt{2|k|}} P_{\leq}(x) \mathrm{e}^{-\mathrm{i} \varepsilon^{\beta} k \cdot y_{i}} \nabla_{y_{i}} \varphi_{m}(x, y)\right\|^{2} \\
& =\left\|\sum_{\ell=1}^{j}\left(|k|+E_{\ell}(x)\right)\left(E_{\ell}(x)-E_{j}(x)+|k|+\mathrm{i} \delta\right)^{-1} \frac{\hat{\rho}(k)}{\sqrt{2|k|}} P_{\ell}(x) \mathrm{e}^{-\mathrm{i} \varepsilon^{\beta} k \cdot y_{i}} \nabla_{y_{i}} \varphi_{m}(x, y)\right\|^{2} \\
& \leq C \sum_{\ell=1}^{j} \int_{0}^{\Lambda_{0}} \frac{\left(E_{\ell}(x)+|k|\right)^{2}|k|}{\left(|k|-\left(E_{j}(x)-E_{\ell}(x)\right)\right)^{2}+\delta^{2}} \mathrm{~d}|k| \leq \frac{C}{\delta} .
\end{aligned}
$$

This shows 62 and 63 . 
To get the bounds for the derivatives first observe that whenever a derivative hits a resolvent, we get

$$
\begin{aligned}
& \partial_{x_{j}}\left(|k|+H_{\mathrm{el}}(x)-E_{j}(x)+\mathrm{i} \delta\right)^{-1} \\
& \quad=\left(|k|+H_{\mathrm{el}}(x)-E_{j}(x)+\mathrm{i} \delta\right)^{-1} \partial_{x_{j}}\left(E_{j}(x)-H_{\mathrm{el}}(x)\right)\left(|k|+H_{\mathrm{el}}(x)-E_{j}(x)+\mathrm{i} \delta\right)^{-1}
\end{aligned}
$$

where $\partial_{x_{j}}\left(E_{j}(x)-H_{\mathrm{el}}(x)\right)$ is uniformly bounded. By Lemma 4 derivatives of $P_{j}$ map into the domain of $H_{\mathrm{el}}$ and thus into the domain of $H_{1}^{\varepsilon}$. Hence, whenever at least one derivative hits $P_{j}$ there will be at most $|\alpha|$ resolvents left and such a term can be estimated by $\delta^{-|\alpha|}$. When all the derivatives hit the resolvent, the worst term has $|\alpha|+1$ resolvents, which can be estimated by $\delta^{-|\alpha|}$ times the norm of $T_{\delta}$.

Corollary 4. Let $\varepsilon^{\frac{1}{2}} \leq \delta \leq 1$. With

$$
P_{\frac{3}{2} \beta}^{\delta}:=T_{\delta}+T_{\delta}^{*}
$$

we have that for $n=0,1$

$$
\left\|P_{\frac{3}{2} \beta}^{\delta}\right\|_{\mathcal{L}\left(D\left(\left(H_{0}^{\varepsilon}\right)^{n}\right)\right)}=\mathcal{O}\left(\delta^{-\frac{1}{2}}\right)
$$

and

$$
\begin{aligned}
&\left\|\tilde{P}_{\text {vac }}^{\varepsilon}-P_{j}^{\varepsilon} \otimes Q_{0}\right\|_{\mathcal{L}\left(D\left(\left(H_{0}^{\varepsilon}\right)^{n}\right)\right)}=\mathcal{O}\left(\varepsilon^{\frac{3}{2} \beta} \delta^{-\frac{1}{2}}\right), \\
&\left\|\tilde{P}_{\text {vac }}^{\varepsilon} \tilde{P}_{\text {vac }}^{\varepsilon}-\tilde{P}_{\text {vac }}^{\varepsilon}\right\|_{\mathcal{L}\left(D\left(\left(H_{0}^{\varepsilon}\right)^{n}\right)\right)}=\mathcal{O}\left(\varepsilon^{3 \beta} \delta^{-1}\right) .
\end{aligned}
$$

Moreover,

$$
\left[P_{\frac{3}{2} \varepsilon}^{\delta}, H_{0}^{\varepsilon}\right]+\left[P_{0} \otimes Q_{0}, H_{1}^{\varepsilon}\right]=\delta^{\frac{1}{2}} \mathcal{T}+R
$$

where

$$
\mathcal{T}=\mathrm{i} \delta^{\frac{1}{2}}\left(T_{\delta}+T_{\delta}^{*}\right)+2 \delta^{-\frac{1}{2}} \varepsilon \nabla_{x}\left(T_{\delta}+T_{\delta}^{*}\right) \cdot \varepsilon \nabla_{x}=\mathcal{O}(1)
$$

in $\mathcal{L}\left(D\left(\left(H_{0}^{\varepsilon}\right)^{n+1}\right), D\left(\left(H_{0}^{\varepsilon}\right)^{n}\right)\right)$ and

$$
\|R\|_{\mathcal{L}\left(D\left(\left(H_{0}^{\varepsilon}\right)^{n}\right)\right)}=\mathcal{O}\left(\varepsilon^{2} \delta^{-\frac{5}{2}}\right) .
$$

Proof. We will use $\delta \geq \varepsilon^{\frac{1}{2}}$ without noting it explicitly. It follows directly from 62 that $\left\|T_{\delta}\right\|=\left\|T_{\delta}^{*}\right\|=\mathcal{O}\left(\delta^{-\frac{1}{2}}\right)$, which yields 65) for $n=0$. For $n=1$ notice that 63), 64), and $T_{\delta}\left(H_{\mathrm{el}}+H_{\mathrm{f}}\right)=T_{\delta} E_{j}$ imply that

$$
\left[H_{0}^{\varepsilon}, T_{\delta}\right]=\left[-\varepsilon^{2} \Delta_{x}+H_{\mathrm{el}}+H_{\mathrm{f}}, T_{\delta}\right]=\left[-\varepsilon^{2} \Delta_{x}, T_{\delta}\right]-H_{1}^{\varepsilon} P_{\mathrm{vac}}+\mathrm{i} \delta T_{\delta}
$$

is $\mathcal{O}(1)$ in $\mathcal{L}\left(D\left(H_{0}^{\varepsilon}\right), \mathcal{H}\right)$ and thus by Lemma 12 also $T_{\delta}=\mathcal{O}\left(\delta^{-\frac{1}{2}}\right)$ in $\mathcal{L}\left(D\left(H_{0}^{\varepsilon}\right)\right)$. In a similar way we find that also $T_{\delta}^{*}$ is $\mathcal{O}\left(\delta^{-\frac{1}{2}}\right)$ in $\mathcal{L}\left(D\left(H_{0}^{\varepsilon}\right)\right)$. The estimate 66 follows immediately from (65). For (67) we note that

$$
P_{\mathrm{vac}} T_{\delta}=0=T_{\delta}^{*} P_{\mathrm{vac}}
$$


because $\left[P_{\text {vac }}, H_{\mathrm{el}}+H_{\mathrm{f}}\right]=0$ and $H_{1}^{\varepsilon}$ creates a photon when applied to $P_{\text {vac. Then we }}$ have

$$
\begin{aligned}
& \left(P^{\varepsilon} \otimes Q_{0}+\varepsilon^{\frac{3}{2} \beta} P_{\frac{3}{2}}^{\delta} \beta\right)\left(P^{\varepsilon} \otimes Q_{0}+\varepsilon^{\frac{3}{2} \beta} P_{\frac{3}{2}}^{\delta} \beta\right)= \\
& \quad=\quad P^{\varepsilon} \otimes Q_{0}+\varepsilon^{\frac{3}{2} \beta}\left(P_{\frac{3}{2} \beta}^{\delta} P^{\varepsilon} \otimes Q_{0}+P^{\varepsilon} \otimes Q_{0} P_{\frac{3}{2} \beta}^{\delta}\right)+\varepsilon^{3 \beta} P_{\frac{3}{2} \beta}^{\delta} P_{\frac{3}{2} \beta}^{\delta} \\
& \quad=\quad P^{\varepsilon} \otimes Q_{0}+\varepsilon^{\frac{3}{2} \beta}\left(P_{\frac{3}{2} \beta}^{\delta} P_{0} \otimes Q_{0}+P_{0} \otimes Q_{0} P_{\frac{3}{2} \beta}^{\delta}\right)+\mathcal{O}\left(\varepsilon^{3 \beta} \delta^{-1}\right) \\
& \stackrel{690}{=} P^{\varepsilon} \otimes Q_{0}+\varepsilon^{\frac{3}{2} \beta} P_{\frac{3}{2} \beta}^{\delta}+\mathcal{O}\left(\varepsilon^{3 \beta} \delta^{-1}\right) .
\end{aligned}
$$

For 68 note that according to 61

$$
\left[P_{\frac{3}{2} \beta}^{\delta}, H_{\mathrm{el}}+H_{\mathrm{f}}\right]+\left[P_{0} \otimes Q_{0}, H_{1}^{\varepsilon}\right]=\mathrm{i} \delta\left(T+T^{*}\right) .
$$

With

$$
\left[P_{\frac{3}{2} \beta}^{\delta},-\varepsilon^{2} \Delta_{x}\right]=2 \varepsilon\left(\nabla_{x} P_{\frac{3}{2} \varepsilon}^{\delta}\right) \cdot \varepsilon \nabla_{x}+\varepsilon^{2}\left(\Delta_{x} P_{\frac{3}{2} \beta}^{\delta}\right)
$$

and $\|R\|_{\mathcal{L}(\mathcal{H})}=\left\|\varepsilon^{2}\left(\Delta_{x} P_{\frac{3}{2} \beta}^{\delta}\right)\right\|_{\mathcal{L}(\mathcal{H})}=\mathcal{O}\left(\varepsilon^{2} \delta^{-\frac{5}{2}}\right)$ we directly obtain 68 for $n=0$. For $n=1$ it suffices to show $\left\|\left[\mathcal{T}, H_{0}^{\varepsilon}\right]\right\|_{\mathcal{L}\left(D\left(H_{0}^{\varepsilon}\right), \mathcal{H}\right)}=\mathcal{O}(1)$. It holds

$$
\left[\mathcal{T}, H_{0}^{\varepsilon}\right]=\mathrm{i} \delta^{\frac{1}{2}}\left[T_{\delta}+T_{\delta}^{*}, H_{0}^{\varepsilon}\right]+\delta^{-\frac{1}{2}} \varepsilon\left[\nabla_{x}\left(T_{\delta}+T_{\delta}^{*}\right), H_{0}^{\varepsilon}\right] .
$$

As shown in the proof of 65 the first term is of order $\delta^{\frac{1}{2}}$. Analogously, it follows that the second term is of order $\varepsilon \delta^{-2}$. Hence, both are $\mathcal{O}(1)$ because of $\varepsilon^{\frac{1}{2}} \leq \delta \leq 1$. Finally $\|R\|_{\mathcal{L}\left(D\left(H_{0}^{\varepsilon}\right)\right)}=\left\|\varepsilon^{2}\left(\Delta_{x} P_{\frac{3}{2} \beta}^{\delta}\right)\right\|_{\mathcal{L}\left(D\left(H_{0}^{\varepsilon}\right)\right)}=\mathcal{O}\left(\varepsilon^{2} \delta^{-\frac{5}{2}}+\varepsilon^{2} \delta^{-\frac{7}{2}}+\varepsilon^{4} \delta^{-\frac{9}{2}}\right)$ follows from Lemma 12 and (64).

Lemma 10. It holds that

$$
\left\|\left[H^{\varepsilon}, \tilde{P}_{\text {vac }}^{\varepsilon}\right]\right\|_{\mathcal{L}\left(D\left(H_{0}^{\varepsilon}\right), \mathcal{H}\right)}=\mathcal{O}\left(\varepsilon+\varepsilon^{\frac{3}{2} \beta} \delta^{\frac{1}{2}}\right)
$$

and

$$
\left[\tilde{P}_{\text {vac }}^{\varepsilon}, H^{\varepsilon}\right] \mathbf{1}_{E+\frac{1}{2}}\left(H_{0}^{\varepsilon}\right)=\varepsilon^{\frac{3}{2} \beta} \delta^{\frac{1}{2}} \mathcal{T} \mathbf{1}_{E+\frac{1}{2}}\left(H_{0}^{\varepsilon}\right)+\mathcal{O}\left(\varepsilon^{\frac{3}{2} \beta+2} \delta^{-\frac{5}{2}}\right)=\mathcal{O}\left(\varepsilon^{\frac{3}{2} \beta} \delta^{\frac{1}{2}}\right)
$$

in $\mathcal{L}(\mathcal{H})$. As a consequence,

$$
\left\|\left[\tilde{\chi}\left(H^{\varepsilon}\right), \tilde{P}_{\mathrm{vac}}^{\varepsilon}\right]\right\|_{\mathcal{L}(\mathcal{H})}=\mathcal{O}\left(\varepsilon^{\frac{3}{2} \beta} \delta^{\frac{1}{2}}\right)
$$

for any smooth $\tilde{\chi}$ with compact support in $\left(-\infty, E+\frac{1}{2}\right)$.

Proof. We first show (71). Due to (68) we only need to check (56)-(59). The estimates (58) and 59) follow from 20 and 119 respectively. Since $\varepsilon^{\frac{3}{2}} \beta \delta^{-\frac{1}{2}}<1$ for $\beta>5 / 6$ and $\delta>\varepsilon^{1 / 2}, P_{\text {vac }}^{\varepsilon}$ is $\mathcal{O}(1)$ in $\mathcal{L}(\overline{\mathcal{H}})$ as well as in $\mathcal{L}\left(D\left(H_{0}^{\varepsilon}\right)\right)$ by $\left.\sqrt[65]{ }\right)$. So $\sqrt{56}$ follows from the fact that $H_{2}^{\varepsilon}$ is uniformly bounded from $D\left(H_{0}^{\varepsilon}\right)$ to $\mathcal{H}$. Since $\varepsilon^{3 \beta} \delta^{-\frac{1}{2}}<\varepsilon^{\frac{3}{2} \beta+1}$ for $\beta>5 / 6$ and $\delta>\varepsilon^{1 / 2}$, (57) also follows from (65). Now (72) directly follows from Lemma 3 .

For (70) we apply exactly the same reasoning as for (71), however, with (21) instead of (20), which worsens the bound.

Now Lemma 8 applied to $\tilde{P}_{\text {vac }}^{\varepsilon}$ with $n=1, \delta \sim \varepsilon^{3 \beta} \delta^{-1}, \delta_{1} \sim \varepsilon+\varepsilon^{\frac{3}{2} \beta} \delta^{\frac{1}{2}}$ and $\beta_{1} \sim \varepsilon^{\frac{3}{2} \beta} \delta^{\frac{1}{2}}$ yields a projector $P_{\text {vac }}^{\varepsilon}$ with all the properties claimed in Propositions 3 and 4 . 


\section{Proofs of Lemmas}

\section{Proof of Lemma 1}

The statement about the potentials is standard. Using for examples the estimates contained in Proposition 1 and in the proof of Proposition 2 of [Ten] we can show easily that

$$
\left\|A^{(i)}\left(\alpha y_{j}\right) \cdot p_{j, y,(i)}\right\|_{\mathcal{L}\left(D_{0}, \mathcal{H}\right)}=\left\|\Phi\left(v_{\alpha y}^{(i)}\right) \cdot p_{j, y,(i)}\right\|_{\mathcal{L}\left(D_{0}, \mathcal{H}\right)} \leq C\left\|v_{\alpha y}^{(i)}\right\|_{\omega}
$$

where

$$
v_{\alpha y}^{(i)}(k, \lambda):=\frac{\hat{\varphi}(\mu k)}{|k|^{1 / 2}} e_{\lambda}^{(i)}(k) \mathrm{e}^{\mathrm{i} k \cdot \alpha y}
$$

and, given a function $f \in L^{2}\left(\mathbb{R}^{3} \otimes \mathbb{C}^{2}\right)$,

$$
\|f\|_{\omega}:=\left(\left\|f|k|^{-1 / 2}\right\|_{L^{2}\left(\mathbb{R}^{3} \otimes \mathbb{C}^{2}\right)}^{2}+\|f\|_{L^{2}\left(\mathbb{R}^{3} \otimes \mathbb{C}^{2}\right)}^{2}\right)^{1 / 2} .
$$

Using these explicit expressions we get then

$$
\left\|A^{(i)}\left(\alpha y_{j}\right) \cdot p_{j, y,(i)}\right\|_{\mathcal{L}\left(D_{0}, \mathcal{H}\right)} \leq C \Lambda \mu^{-1}=C \frac{\Lambda}{2 m_{\mathrm{el}} \alpha^{2}}=C \Lambda_{0} .
$$

In the same way we have

$$
\left\|: A\left(\alpha y_{j}\right)^{2}:\right\|_{\mathcal{L}\left(D_{0}, \mathcal{H}\right)}^{2} \leq C\left\|v_{\alpha y}^{(i)}\right\|_{\omega}^{2} \leq C\left(\Lambda \mu^{-1}\right)^{2}=C \Lambda_{0}^{2} .
$$

Identical results hold for the coefficients of the Hamiltonian containing the nuclear coordinates, so all the coefficients in $H^{\varepsilon}$ can be bounded with an $\varepsilon$-independent bound in terms of $H_{0}^{\varepsilon}$ or $H_{\text {free }}^{\varepsilon}$.

\section{Proof of Lemma 2}

Since $H_{f}$ is nonnegative and since $H_{\mathrm{mol}}^{\varepsilon} \otimes 1$ and $1 \otimes H_{\mathrm{f}}$ commute, we have that

$$
\left(H_{\mathrm{mol}}^{\varepsilon} \otimes 1\right)^{n} \leq\left(H_{\mathrm{mol}}^{\varepsilon} \otimes 1+1 \otimes H_{\mathrm{f}}\right)^{n}=\left(H_{0}^{\varepsilon}\right)^{n}
$$

for any $n \in \mathbb{N}$. To estimate tensor product operators in $\mathcal{L}\left(D_{0}^{n}\right)$, the following characterization of this operator norm will be useful.

Lemma 11. Let $(H, D(H))$ be self-adjoint and

$$
D^{n}:=\left\{\psi \in D(H) \mid H^{k} \psi \in D(H) \text { for } k=1, \ldots n-1\right\}
$$

be equipped with the graph norm

$$
\|\psi\|_{D^{n}}:=\sum_{j=0}^{n}\left\|H^{j} \psi\right\| .
$$


Then $\left(D^{n},\|\cdot\|_{D^{n}}\right)$ is a Banach space,

$$
D_{R}^{n}:=\left\{(H+\mathrm{i})^{-n} \psi \mid \psi \in \mathcal{H}\right\}=D^{n}
$$

and for $A \in \mathcal{L}\left(D^{n}, D^{m}\right)$ the operator norm $\|A\|_{\mathcal{L}\left(D^{n}, D^{m}\right)}$ is equivalent to the norm

$$
\|A\|_{R(n, m)}:=\sum_{j=0}^{m}\left\|H^{j} A(H+\mathrm{i})^{-n}\right\|_{\mathcal{L}(\mathcal{H})} .
$$

More precisely, there are constants $C_{n}$ depending only on $n$ (not on $H$ or $A$ ), such that

$$
\frac{1}{1+m}\|A\|_{R(n, m)} \leq\|A\|_{\mathcal{L}\left(D^{n}, D^{m}\right)} \leq C_{n}\|A\|_{R(n, m)} .
$$

Proof. Since $H$ is self-adjoint, it is closed and therefore $\left(D^{n},\|\cdot\|_{D^{n}}\right)$ is a Banach space. Let $\psi \in D^{n}$, then $(H+\mathrm{i})^{n} \psi \in \mathcal{H}$ and thus $\psi \in D_{R}^{n}$. Let conversely $\psi=(H+\mathrm{i})^{-n} \phi \in D_{R}^{n}$, then $H^{k} \psi \in \mathcal{H}$ for $k \leq n$ since $H(H+\mathrm{i})^{-1} \in \mathcal{L}(\mathcal{H})$. For the norms observe that

$$
\begin{aligned}
\|A \psi\|_{D^{m}} & =\sum_{j=0}^{m}\left\|H^{j} A \psi\right\|=\sum_{j=0}^{m}\left\|H^{j} A(H+\mathrm{i})^{-n}(H+\mathrm{i})^{n} \psi\right\| \\
& \leq \sum_{j=0}^{m}\left\|H^{j} A(H+\mathrm{i})^{-n}\right\|_{\mathcal{L}(\mathcal{H})}\left\|(H+\mathrm{i})^{n} \psi\right\| \leq C_{n}\|A\|_{R(n, m)}\|\psi\|_{D^{n}}
\end{aligned}
$$

and thus

$$
\|A\|_{\mathcal{L}\left(D^{n}, D^{m}\right)} \leq C_{n}\|A\|_{R(n, m)} .
$$

Conversely for $j \leq m$

$$
\begin{aligned}
\left\|H^{j} A(H+\mathrm{i})^{-n} \psi\right\| & \leq\left\|A(H+\mathrm{i})^{-n} \psi\right\|_{D^{m}} \leq\|A\|_{\mathcal{L}\left(D^{n}, D^{m}\right)}\left\|(H+\mathrm{i})^{-n} \psi\right\|_{D^{n}} \\
& \leq\|A\|_{\mathcal{L}\left(D^{n}, D^{m}\right)}\|\psi\|,
\end{aligned}
$$

where we use $\left\|(H+\mathrm{i})^{-1}\right\|_{\mathcal{L}\left(D^{j}, D^{j+1}\right)}=1$. Thus

$$
\|A\|_{R(n, m)} \leq(m+1)\|A\|_{\mathcal{L}\left(D^{n}, D^{m}\right)} .
$$

So the $\mathcal{L}\left(D_{0}^{n}, D_{0}^{m}\right)$-norm of an operator $B \otimes 1$ for $m \leq n$ is estimated by

$$
\|B \otimes 1\|_{\mathcal{L}\left(D_{0}^{n}, D_{0}^{m}\right)} \leq C_{n} \sum_{j=0}^{m}\left\|\left(H_{0}^{\varepsilon}\right)^{j}(B \otimes 1)\left(H_{0}^{\varepsilon}+\mathrm{i}\right)^{-n}\right\| .
$$

This will turn out useful, since for bounded operators on Hilbert spaces $\|A \otimes B\|=$ 
$\|A\| \cdot\|B\|$. Let's look at a single term in the sum more closely,

$$
\begin{gathered}
\left(H_{0}^{\varepsilon}\right)^{j}(B \otimes 1)\left(H_{0}^{\varepsilon}+\mathrm{i}\right)^{-n}=\left(H_{\mathrm{mol}}^{\varepsilon} \otimes 1+1 \otimes H_{\mathrm{f}}\right)^{j}(B \otimes 1)\left(H_{0}^{\varepsilon}+\mathrm{i}\right)^{-n} \\
=\sum_{\ell=0}^{j}\left(\begin{array}{l}
j \\
\ell
\end{array}\right)\left(\left(H_{\mathrm{mol}}^{\varepsilon}\right)^{\ell} \otimes 1\right)\left(1 \otimes H_{f}^{j-\ell}\right)(B \otimes 1)\left(H_{0}^{\varepsilon}+\mathrm{i}\right)^{-n} \\
=\sum_{\ell=0}^{j}\left(\begin{array}{l}
j \\
\ell
\end{array}\right)\left(\left(H_{\mathrm{mol}}^{\varepsilon}\right)^{\ell} B \otimes H_{f}^{j-\ell}\right)\left(\left(H_{\mathrm{mol}}^{\varepsilon}+\mathrm{i}\right)^{-(\ell+n-j)} \otimes\left(H_{\mathrm{f}}+\mathrm{i}\right)^{-(j-\ell)}\right) \times \\
\times\left(\left(H_{\mathrm{mol}}^{\varepsilon}+\mathrm{i}\right)^{\ell+n-j} \otimes\left(H_{\mathrm{f}}+\mathrm{i}\right)^{j-\ell}\right)\left(H_{0}^{\varepsilon}+\mathrm{i}\right)^{-n} \\
=\sum_{\ell=0}^{j}\left(\begin{array}{l}
j \\
\ell
\end{array}\right)\left(\left(H_{\mathrm{mol}}^{\varepsilon}\right)^{\ell} B\left(H_{\mathrm{mol}}^{\varepsilon}+\mathrm{i}\right)^{-(\ell+n-j)} \otimes H_{f}^{j-\ell}\left(H_{\mathrm{f}}+\mathrm{i}\right)^{-(j-\ell)}\right) \times \\
\times\left(\left(H_{\mathrm{mol}}^{\varepsilon}+\mathrm{i}\right)^{\ell+n-j} \otimes\left(H_{\mathrm{f}}+\mathrm{i}\right)^{j-\ell}\right)\left(H_{0}^{\varepsilon}+\mathrm{i}\right)^{-n} .
\end{gathered}
$$

Since

$$
\left(\left(H_{\mathrm{mol}}^{\varepsilon}+\mathrm{i}\right)^{\ell+n-j} \otimes\left(H_{\mathrm{f}}+\mathrm{i}\right)^{j-\ell}\right)\left(H_{0}^{\varepsilon}+\mathrm{i}\right)^{-n} \text { and } H_{\mathrm{f}}^{j-\ell}\left(H_{\mathrm{f}}+\mathrm{i}\right)^{-(j-\ell)}
$$

are bounded uniformly in $\varepsilon$ due to $(73)$, it suffices to control terms of the form

$$
\left(H_{\mathrm{mol}}^{\varepsilon}\right)^{\ell} B\left(H_{\mathrm{mol}}^{\varepsilon}+\mathrm{i}\right)^{-(\ell+n-j)} .
$$

By Lemma 11 these are controlled again in terms of $\|B\|_{\mathcal{L}\left(D_{\text {mol }}^{\ell+n-j}, D_{\text {mol }}^{\ell}\right)}$.

\section{Proof of Lemma 3}

The assumption $\|A\|_{\mathcal{L}\left(D_{0}, \mathcal{H}\right)} \leq \delta<1$ implies that for $\psi \in D$

$$
\|A \psi\| \leq \delta\|\psi\|_{D_{0}}=\delta\left(\left\|H_{0} \psi\right\|+\|\psi\|\right)
$$

and thus $A$ is $H_{0}$-bounded with relative bound smaller than 1 . The equivalence of the norms follows from

$$
\|\psi\|_{D_{H}}=\|H \psi\|+\|\psi\| \leq\left\|H_{0} \psi\right\|+\|\psi\|+\|A \psi\| \leq\|\psi\|_{D_{0}}(1+\delta)
$$

and

$$
\|\psi\|_{D_{0}}=\left\|H_{0} \psi\right\|+\|\psi\| \leq\|H \psi\|+\|\psi\|+\|A \psi\| \leq\|\psi\|_{D_{H}}+\delta\|\psi\|_{D_{0}} .
$$

The last claim follows from the Helffer-Sjöstrand formula

$$
\tilde{\chi}(H)=\frac{1}{\pi} \int_{\mathbb{C}} \partial_{\bar{z}} \hat{\chi}(z)(H-z)^{-1} \mathrm{~d} z
$$

where $\hat{\chi}$ is an appropriate almost-analytic extension of $\tilde{\chi}$, and the resolvent formula

$$
\begin{aligned}
\left\|\left(H_{0}-z\right)^{-1}-(H-z)^{-1}\right\|_{\mathcal{L}(\mathcal{H}, D)} & =\left\|(H-z)^{-1} A\left(H_{0}-z\right)^{-1}\right\|_{\mathcal{L}(\mathcal{H}, D)} \\
& \leq\left\|(H-z)^{-1}\right\|_{\mathcal{L}(\mathcal{H}, D)}\|A\|_{\mathcal{L}(D, \mathcal{H})}\left\|\left(H_{0}-z\right)^{-1}\right\|_{\mathcal{L}(\mathcal{H}, D)} \\
& \leq \delta(1+\delta)\left(1+\frac{|z|+1}{|\operatorname{Im} z|}\right)^{2}
\end{aligned}
$$




\section{Proof of Lemma 4}

Due to the smearing of the nuclear charge it holds $V_{\mathrm{nn}}, V_{\mathrm{en}} \in C_{\mathrm{b}}^{\infty}\left(\mathbb{R}^{3 l}, C_{\mathrm{b}}^{\infty}\left(\mathbb{R}^{3 r}\right)\right)$. Note that

$$
\left[\nabla_{x},\left(H_{\mathrm{el}}(x)-z\right)^{-1}\right]=\left(H_{\mathrm{el}}(x)-z\right)^{-1}\left(\nabla_{x} V_{\mathrm{nn}}(x)+\nabla_{x} V_{\mathrm{en}}(x)\right)\left(H_{\mathrm{el}}(x)-z\right)^{-1} .
$$

Thus the mapping $x \mapsto\left(H_{\mathrm{el}}(x)-z\right)^{-1}$ is in $C_{\mathrm{b}}^{1}\left(\mathbb{R}^{3 l}, \mathcal{L}\left(\mathcal{H}_{\mathrm{el}}\right)\right)$. Since $E_{j}$ is separated by a gap, the projection $P_{j}(x)$ associated to $E_{j}(x)$ is given via the Riesz formula:

$$
P_{j}(x)=\frac{\mathrm{i}}{2 \pi} \oint_{\gamma(x)}\left(H_{\mathrm{el}}(x)-z\right)^{-1} \mathrm{~d} z
$$

where $\gamma(x)$ is positively oriented closed curve encircling $E_{j}(x)$ once. It can be chosen independent of $x$ locally because the gap condition is uniform. Therefore $\left(H_{\mathrm{el}}(\cdot)-z\right)^{-1} \in$ $C_{\mathrm{b}}^{1}\left(\mathbb{R}^{3 l}, \mathcal{L}\left(\mathcal{H}_{\mathrm{el}}\right)\right)$ entails that $P_{j} \in C_{\mathrm{b}}^{1}\left(\mathbb{R}^{3 l}, \mathcal{L}\left(\mathcal{H}_{\mathrm{el}}\right)\right)$. By

$$
E_{j}(x) P_{j}(x)=H_{\mathrm{el}}(x) P_{j}(x)=\frac{\mathrm{i}}{2 \pi} \oint_{\gamma(x)} z\left(H_{\mathrm{el}}(x)-z\right)^{-1} \mathrm{~d} z
$$

we obtain $E_{j} P_{j} \in C_{\mathrm{b}}^{1}\left(\mathbb{R}^{3 l}, \mathcal{L}\left(\mathcal{H}_{\mathrm{el}}\right)\right)$. Then $E_{j}=\operatorname{tr}_{L^{2}\left(\mathbb{R}^{3 s}\right)}\left(E_{j} P_{j}\right) \in C_{\mathrm{b}}^{1}\left(\mathbb{R}^{3 l}\right)$. For it holds

$$
\begin{aligned}
\nabla_{x} \operatorname{tr}\left(E_{j} P_{j}\right) & =\nabla_{x} \operatorname{tr}\left(\left(E_{j} P_{j}\right) P_{j}\right)=\operatorname{tr}\left(\left(\nabla_{x} E_{j} P_{j}\right) P_{j}+\left(E_{j} P_{j}\right) \nabla_{x} P_{j}\right) \\
& =\operatorname{tr}\left(\left(\nabla_{x} E_{j} P_{j}\right) P_{j}\right)+\operatorname{tr}\left(\left(E_{j} P_{j}\right) \nabla_{x} P_{j}\right)<\infty
\end{aligned}
$$

because $P_{j}$ and $E_{j} P_{j}$ are trace-class operators and the product of a trace-class operator and a bounded operator is again a trace-class operator (see e.g. $\mathrm{ReSi}_{1}$, Theorem VI.19). The argument for higher derivatives goes along the same lines.

For the last claim we observe that $H_{\mathrm{el}}^{n} \partial_{x}^{\alpha} P_{j}$ is bounded for any $\alpha \in \mathbb{N}_{0}^{3 l}$ and any $n \in \mathbb{N}_{0}$. Since $H_{\mathrm{el}}, E_{j}$ and $P_{j}$ have bounded and smooth derivatives, this can be easily seen inductively by differentiating the identity

$$
0=\left(H_{\mathrm{el}}-E_{j}\right)^{n} P_{j}
$$

\section{Proof of Lemma 5}

We proceed by induction. For $m=1$, i.e. $|\alpha| \leq 2$, we have by standard elliptic estimates that $\varepsilon^{|\alpha|} \partial_{x}^{\alpha}$ is relatively bounded by $-\varepsilon^{2} \Delta_{x}$. Now $H_{\text {mol }}^{\varepsilon}$ has the form

$$
H_{\mathrm{mol}}^{\varepsilon}=-\varepsilon^{2} \Delta_{x} \otimes 1+H_{\mathrm{el}}(x)=-\varepsilon^{2} \Delta_{x} \otimes 1+1 \otimes \underbrace{\left(-\Delta_{y}+V_{\mathrm{ee}}(y)\right)}_{=: H_{\mathrm{e}, 0} \geq 0}+V_{\mathrm{en}}(x, y),
$$

where $V_{\text {en }}$ is bounded with bounded derivatives. Hence

$$
\left\|-\varepsilon^{2} \Delta_{x} \psi\right\| \leq\left\|H_{\mathrm{mol}}^{\varepsilon} \psi\right\|+\left\|V_{\mathrm{en}}\right\|_{\infty}\|\psi\|
$$

and thus

$$
\left\|A_{\alpha} \varepsilon^{\alpha} \partial_{x}^{\alpha} \psi\right\| \leq\left\|A_{\alpha}\right\|\left\|\varepsilon^{\alpha} \partial_{x}^{\alpha} \psi\right\| \leq C\left(\left\|H_{\mathrm{mol}}^{\varepsilon} \psi\right\|+\|\psi\|\right)=C\|\psi\|_{D\left(H_{\mathrm{mol}}^{\varepsilon}\right)}
$$


with a constant $C$ independent of $\varepsilon$.

Now assume that we proved the assertion for operators of order $n-1$ and let $|\alpha|=n$ and $m=\lceil n / 2\rceil$. Then $A(x) \varepsilon^{|\alpha|} \partial_{x}^{\alpha}$ is relatively bounded by $\left(-\varepsilon^{2} \Delta_{x}\right)^{m}$ again by standard elliptic estimates. Using the induction hypothesis we find that

$$
\begin{aligned}
\left\|\left(\varepsilon^{2} \Delta_{x}\right)^{m} \psi\right\| & \leq C\left(\left\|H_{\mathrm{mol}}^{\varepsilon}\left(\varepsilon^{2} \Delta_{x}\right)^{m-1} \psi\right\|+\left\|\left(\varepsilon^{2} \Delta_{x}\right)^{m-1} \psi\right\|\right) \\
& \leq C\left(\left\|\left(\varepsilon^{2} \Delta_{x}\right)^{m-1} H_{\mathrm{mol}}^{\varepsilon} \psi\right\|+\left\|\left[H_{\mathrm{mol}}^{\varepsilon},\left(\varepsilon^{2} \Delta_{x}\right)^{m-1}\right] \psi\right\|+\left\|\left(\varepsilon^{2} \Delta_{x}\right)^{m-1} \psi\right\|\right) \\
& \leq C\|\psi\|_{D\left(\left(H_{\mathrm{mol}}^{\varepsilon}\right)^{m}\right)},
\end{aligned}
$$

since $\left(\varepsilon^{2} \Delta_{x}\right)^{m-1}$ and $\left[H_{\mathrm{mol}}^{\varepsilon},\left(\varepsilon^{2} \Delta_{x}\right)^{m-1}\right]$ are both differential operators of order at most $2 m-2 \leq n-1$.

For the second claim note that

$$
\begin{aligned}
& \left\|\left(H_{\mathrm{mol}}^{\varepsilon}\right)^{k} A_{\alpha} \varepsilon^{\alpha} \partial_{x}^{\alpha} \psi\right\| \\
& \quad \leq\left\|\left[\left(H_{\mathrm{mol}}^{\varepsilon}\right)^{k}, A_{\alpha}\right] \varepsilon^{\alpha} \partial_{x}^{\alpha} \psi\right\|+\left\|A_{\alpha}\left[\left(H_{\mathrm{mol}}^{\varepsilon}\right)^{k}, \varepsilon^{\alpha} \partial_{x}^{\alpha}\right] \psi\right\|+\left\|A_{\alpha} \varepsilon^{\alpha} \partial_{x}^{\alpha}\left(H_{\mathrm{mol}}^{\varepsilon}\right)^{k} \psi\right\| \\
& \quad \leq C\|\psi\|_{D\left(\left(H_{\mathrm{mol}}^{\varepsilon}\right)^{m+k}\right)}
\end{aligned}
$$

since $\left[\left(H_{\mathrm{mol}}^{\varepsilon}\right)^{k}, A_{\alpha}\right] \varepsilon^{\alpha} \partial_{x}^{\alpha}$ and $\left[\left(H_{\mathrm{mol}}^{\varepsilon}\right)^{k}, \varepsilon^{\alpha} \partial_{x}^{\alpha}\right]$ are admissible of order $2 k-1+n \leq 2(k+m)$.

\section{Proof of Lemma 6}

All the operators appearing are differential operators with coefficients $A_{\alpha}$ that are composed of derivatives of $P_{0}$ and $R$, i.e. of $\partial_{x}^{\alpha} P_{0}$ and $\partial_{x}^{\beta} R$. So they are all admissible in the sense of Lemma 5. It remains to show that also commutators of the coefficients $A_{\alpha}$ with $\left(H_{\text {mol }}^{\varepsilon}\right)^{k}$ are admissible, which in turn follows if $H_{\mathrm{e}}^{k} \partial_{x}^{\beta} A_{\alpha}$ is bounded for any $\beta \in \mathbb{N}_{0}^{3 l}$. Now according to Lemma $4 \partial_{x}^{\beta} P_{0}(x) \in \mathcal{L}\left(\mathcal{H}_{\mathrm{el}}, D\left(H_{\mathrm{el}}^{n}\right)\right)$ for any $n$ and clearly also $\partial_{x}^{\beta} R(x) \in \mathcal{L}\left(D\left(H_{\mathrm{el}}^{n}\right)\right)$ for any $n$. Since every coefficient $A_{\alpha}$ appearing in the construction contains at least one factor of the type $\partial_{x}^{\beta} P_{0}$, the claim follows.

\section{Proof of Lemma 7}

First take any $\phi \in C_{0}^{\infty}(\mathbb{R})$ and $\hat{\phi}$ an appropriate almost analytic extension. Then the Helffer-Sjöstrand formula implies

$$
\begin{aligned}
& \left\|[\phi(H), A] \mathbf{1}_{I}(H)\right\|_{\mathcal{L}\left(\mathcal{H}, D\left(H^{n}\right)\right)} \\
& \quad=\left\|\frac{1}{\pi} \int_{\mathbb{C}} \partial_{\bar{z}} \hat{\phi}(z)(H-z)^{-1}[A, H] \mathbf{1}_{I}(H)(H-z)^{-1} \mathrm{~d} z\right\|_{\mathcal{L}\left(\mathcal{H}, D\left(H^{n}\right)\right)} \\
& \quad \leq \frac{\delta}{\pi} \int_{\mathbb{C}}\left|\partial_{\bar{z}} \hat{\phi}(z)\right|\left\|(H-z)^{-1}\right\|_{\mathcal{L}\left(D\left(H^{n}\right)\right)}\left\|(H-z)^{-1}\right\|_{\mathcal{L}(\mathcal{H})} \mathrm{d} z \\
& \quad \leq \frac{\delta}{\pi} \int_{\mathbb{C}}\left|\partial_{\bar{z}} \hat{\phi}(z)\right| \frac{1}{|\operatorname{Im}(z)|^{2}} \mathrm{~d} z \leq C_{\phi} \delta .
\end{aligned}
$$

Taking the adjoint shows that also $\left\|\mathbf{1}_{I}(H)[\phi(H), A]\right\|_{\mathcal{L}(\mathcal{H})} \leq C_{\phi} \delta$. With the bound $\left\|\mathbf{1}_{I}(H)\right\|_{\mathcal{L}\left(\mathcal{H}, D\left(H^{n}\right)\right)} \leq C_{n}$ we get also

$$
\left\|\mathbf{1}_{I}(H)[\phi(H), A]\right\|_{\mathcal{L}\left(\mathcal{H}, D\left(H^{n}\right)\right)} \leq C_{n} C_{\phi} \delta .
$$


Now choose $\chi \in C_{0}^{\infty}(\mathbb{R})$ with $\operatorname{supp} \chi \subset I$ and $\left.\chi\right|_{\tilde{I}}=1$. This implies $\tilde{\chi}=\tilde{\chi} \mathbf{1}_{I}(H)$, $\chi=\chi \mathbf{1}_{I}(H)$ and $\tilde{\chi} \chi=\tilde{\chi}$. Using the above estimate for $\phi=\chi$ and $\phi=\tilde{\chi}$, we get

$$
\begin{aligned}
\|\tilde{\chi}(H) A-A \tilde{\chi}(H)\| & =\|\tilde{\chi}(H) A-A \chi(H) \tilde{\chi}(H)\| \leq\|\tilde{\chi}(H) A-\chi(H) A \tilde{\chi}(H)\|+C_{\chi} \delta \\
& \leq\left(C_{\chi}+C_{n} C_{\tilde{\chi}}\right) \delta
\end{aligned}
$$

\section{Proof of Lemma 8}

We first state another lemma that will be used in the proof.

Lemma 12. Let $(H, D(H))$ be a self-adjoint operator and equip the domains $D^{n}:=$ $D\left(H^{n}\right)$ with the graph norms $\|\psi\|_{D^{n}}:=\sum_{i=0}^{n}\left\|H^{i} \psi\right\|$ and let $N \in \mathbb{N}$. If $A \in \mathcal{L}(\mathcal{H})$ satisfies

$$
\|[A, H]\|_{\mathcal{L}\left(D^{n}, D^{n-1}\right)} \leq \delta_{n}
$$

for all $1 \leq n \leq N$, then

$$
\|A\|_{\mathcal{L}\left(D^{n}\right)} \leq n\|A\|+\sum_{i=1}^{n} \delta_{i}
$$

for all $1 \leq n \leq N$. Moreover, if in addition

$$
\left\|(A-z)^{-1}\right\|_{\mathcal{L}(\mathcal{H})} \leq \alpha
$$

and $\delta_{n}<\frac{1}{2} \frac{1}{2^{2 n} \alpha}$, then

$$
\left\|(A-z)^{-1}\right\|_{\mathcal{L}\left(D^{n}\right)} \leq 2^{2 n} \alpha
$$

for all $n \leq N$.

Proof. We proceed by induction.

$$
\begin{aligned}
\|A \psi\|_{D^{n}} & =\sum_{i=0}^{n}\left\|H^{i} A \psi\right\| \leq\|A \psi\|+\sum_{i=0}^{n-1}\left\|H^{i} A H \psi\right\|+\sum_{i=0}^{n-1}\left\|H^{i}[H, A] \psi\right\| \\
& =\|A \psi\|+\|A H \psi\|_{D^{n-1}}+\|[H, A] \psi\|_{D^{n-1}} \\
& \leq\|A\|\|\psi\|+\|A\|_{\mathcal{L}\left(D^{n-1}\right)}\|\psi\|_{D^{n}}+\|[H, A]\|_{\mathcal{L}\left(D^{n}, D^{n-1}\right)}\|\psi\|_{D^{n}}
\end{aligned}
$$

and thus

$$
\|A\|_{\mathcal{L}\left(D^{n}\right)} \leq\|A\|_{\mathcal{L}\left(D^{n-1}\right)}+\|A\|+\delta_{n} .
$$

Since for $n=1$ the computation yields $\|A\|_{\mathcal{L}(D)} \leq\|A\|+\delta_{1}$, this implies (74). For (75) we proceed analogously and abbreviate $R:=(A-z)^{-1}$.

$$
\begin{aligned}
\|R \psi\|_{D^{n}} & =\sum_{i=0}^{n}\left\|H^{i} R \psi\right\| \leq\|R \psi\|+\sum_{i=0}^{n-1}\left\|H^{i} R H \psi\right\|+\sum_{i=0}^{n-1}\left\|H^{i}[H, R] \psi\right\| \\
& =\|R \psi\|+\|R H \psi\|_{D^{n-1}}+\|R[A, H] R \psi\|_{D^{n-1}} \\
& \leq\|R\|\|\psi\|+\|R\|_{\mathcal{L}\left(D^{n-1}\right)}\|\psi\|_{D^{n}}+\|R\|_{\mathcal{L}\left(D^{n-1}\right)} \delta_{n}\|R\|_{\mathcal{L}\left(D^{n}\right)}\|\psi\|_{D^{n}}
\end{aligned}
$$


and thus

$$
\|R\|_{\mathcal{L}\left(D^{n}\right)} \leq \frac{\|R\|+\|R\|_{\mathcal{L}\left(D^{n-1}\right)}}{1-\delta_{n}\|R\|_{\mathcal{L}\left(D^{n-1}\right)}}
$$

For $n=1$ the this yields $\|R\|_{\mathcal{L}(D)} \leq 4 \alpha$ if $1-\delta_{1} \alpha>\frac{1}{2}$ and by induction one obtains 75 .

Sine $\tilde{Q}$ is self-adjoint in $\mathcal{L}(\mathcal{H}), 50$ implies that the spectrum of $\tilde{Q}$ is located in balls of radius $\delta_{1}$ around 0 and 1 . Thus, for $\delta<\frac{1}{2}$ the curve $\gamma:[0,2 \pi) \rightarrow \mathbb{C}, \gamma(\theta)=1+\frac{1}{2} \mathrm{e}^{\mathrm{i} \theta}$, is contained in the resolvent set of $\tilde{Q} \in \mathcal{L}(\mathcal{H})$ and we can define

$$
Q:=\frac{\mathrm{i}}{2 \pi} \oint_{\gamma}(\tilde{Q}-z)^{-1} \mathrm{~d} z
$$

as a bounded operator in $\mathcal{L}(\mathcal{H})$. Note that $Q$ is just the spectral projection of $\tilde{Q}$ related to the spectrum near 1 . For simplicity write $R(z):=(\tilde{Q}-z)^{-1}$ and assume $\delta<\frac{1}{4}$. Then for $z \in \gamma$ we have $\|R(z)\| \leq 4$ and by Lemma 12 for $\delta_{n}<\frac{1}{2} \frac{1}{2^{2 n}}$ also

$$
\|R(z)\|_{\mathcal{L}\left(D^{n}\right)} \leq 4 \cdot 2^{2 n}=4^{n+1}
$$

is uniformly bounded on $\gamma$. Hence $\|Q\|_{\mathcal{L}\left(D^{n}\right)} \leq 4^{n+1}$ for all $n \leq N$. The fact that $\tilde{Q}-Q$ has spectrum only in a ball around 0 of size $\delta$ implies

$$
\|\tilde{Q}-Q\|_{\mathcal{L}(\mathcal{H})} \leq \delta .
$$

To estimate the difference also in $\mathcal{L}\left(D^{n}\right)$ we use Nenciu's formula Nen

$$
Q-\tilde{Q}=\frac{\mathrm{i}}{2 \pi} \oint_{\gamma} \frac{R(z)-R(1-z)}{1-z} \mathrm{~d} z(\tilde{Q} \tilde{Q}-\tilde{Q}) .
$$

Now (50) together with $(76)$ implies the second part of $(52)$ with $C_{n}=8 \cdot 4^{n+1}$. From

$$
[H, Q]=\frac{\mathrm{i}}{2 \pi} \oint_{\gamma} R(z)[H, \tilde{Q}] R(z) \mathrm{d} z,
$$

and (51) it follows that

$$
\|[H, Q]\|_{\mathcal{L}\left(D^{n}, D^{n-1}\right)} \leq C_{n} \delta_{n}
$$

with $C_{n}=4^{2 n+1}$. From now on we will not keep track of the exact value of $C_{n}$ and increase it as necessary in the following steps. But it should always be noted that it depends only on $n$.

Now pick $\tilde{\chi} \in C_{0}^{\infty}(\mathbb{R})$ with support in $(e-1, E+1)$ and with $\tilde{\chi} \mathbf{1}_{E+\frac{1}{2}}=\mathbf{1}_{E+\frac{1}{2}}$. Then (53) together with Lemma 7 implies that there is a constant $C$ depending only on $\tilde{\chi}$, which in turn can be fixed given $E$, such that

$$
\|[\tilde{\chi}(H), \tilde{Q}]\|_{\mathcal{L}\left(\mathcal{H}, D^{n}\right)} \leq C \beta_{1} .
$$

Thus for $z \in \gamma$

$$
\|[R(z), \tilde{\chi}(H)]\|_{\mathcal{L}\left(\mathcal{H}, D^{n}\right)}=\|R(z)[\tilde{\chi}(H), \tilde{Q}] R(z)\|_{\mathcal{L}\left(\mathcal{H}, D^{n}\right)} \leq C_{n} C \beta_{1},
$$


which shows that

$$
\begin{aligned}
{[H, Q] \mathbf{1}_{\mathrm{E}+\frac{1}{2}}(H)=} & \frac{\mathrm{i}}{2 \pi} \oint_{\gamma} R(z)[H, \tilde{Q}] R(z) \mathrm{d} z \tilde{\chi}(H) \mathbf{1}_{\mathrm{E}+\frac{1}{2}}(H) \\
= & \frac{\mathrm{i}}{2 \pi} \oint_{\gamma} R(z)[H, \tilde{Q}] \tilde{\chi}(H) R(z) \mathrm{d} z \mathbf{1}_{\mathrm{E}+\frac{1}{2}}(H) \\
& +\frac{\mathrm{i}}{2 \pi} \oint_{\gamma} R(z)[H, \tilde{Q}][R(z), \tilde{\chi}(H)] \mathrm{d} z \mathbf{1}_{\mathrm{E}+\frac{1}{2}}(H)
\end{aligned}
$$

implies

$$
\left\|[H, Q] \mathbf{1}_{\mathrm{E}+\frac{1}{2}}(H)\right\|_{\mathcal{L}\left(\mathcal{H}, D^{n}\right)} \leq C_{n} C_{E} \beta_{1}
$$

Finally we get (55) using again Nenciu's formula and (54).

\section{Acknowledgements}

We are grateful to Luca Tenuta for his engagement in the initial phase of this project. We thank Jürg Fröhlich, Marcel Griesemer, Christian Hainzl, Michael Sigal, Herbert Spohn, Hans-Michael Stiepan and Jan-Eric Sträng for helpful remarks and stimulating discussions. This work was supported by the German Science Foundation (DFG) and by the German Israeli Foundation (GIF).

\section{References}

[AFFS] W.K. Abou Salem, J. Faupin, J. Fröhlich and I. M. Sigal. On theory of resonances in non-relativistic QED and related models, Adv. in Appl. Math. 43, 201230 (2009).

[BFS] V. Bach, J. Fröhlich and I.M. Sigal. Spectral Analysis for Systems of Atoms and Molecules Coupled to the Quantized Radiation Field, Commun. Math. Phys. 207, 249-290 (1999).

[BGT] V. Betz, B. Goddard and S. Teufel. Superadiabatic transitions in quantum molecular dynamics, Proc. R. Soc. Lond. Ser. A Math. Phys. Eng. Sci. 465, 3553-3580 (2009).

[BeGo] V. Betz and B. Goddard. Accurate prediction of non-adiabatic transitions through avoided crossings, Phys. Rev. Lett. 103, 213001 (2009).

[Fa] J. Faupin. Resonances of the Confined Hydrogen Atom and the Lamb-Dicke Effect in Non-Relativistic QED, Ann. Henri Poincaré 9, 743-773 (2008).

[GLL] M. Griesemer, E.H. Lieb and M. Loss. Ground states in non-relativistic quantum electrodynamics, Invent. Math. 145, 557-595 (2001). 
[HaSe] C. Hainzl and R. Seiringer. Mass renormalization and energy level shift in nonrelativistic QED, Adv. Theor. Math. Phys. 6, 847-871 (2002).

[HaJo $\left.{ }_{1}\right]$ G.A. Hagedorn and A. Joye, A time-dependent Born-Oppenheimer approximation with exponentially small error estimates, Comm. Math. Phys. 223, 583-626 (2001).

[HaJo $\left.\mathrm{H}_{2}\right]$ G.A. Hagedorn and A. Joye, Determination of non-adiabatic scattering wave functions in a Born-Oppenheimer model, Ann. Henri Poincaré 6, 937-990 (2005).

[HiSi] P.D. Hislop and I.M. Sigal. Introduction to Spectral Theory with Applications to Schrödinger Operators, Applied Mathematical Sciences 113, Springer (1996).

[HHH] D. Hasler, I. Herbst and M. Huber. On the Lifetime of Quasi-stationary States in Non-Relativistic QED, Ann. Henri Poincaré 9, 1005-1028 (2008).

[Hu] W. Hunziker. Distortion analyticity and molecular resonance curves, Ann. Inst. H. Poincaré 45, 339-358 (1986).

[JaLu] T. Jahnke and C. Lubich. Error Bounds for Exponential Operator Splittings, BIT 40, 735-744 (2000).

[LaTe] C. Lasser and S. Teufel. Propagation through conical crossings: an asymptotic semigroup, Comm. Pure Appl. Math. 58, 1188-1230 (2005).

[LiLo] E.H. Lieb and M. Loss. Existence of Atoms and Molecules in Non-Relativistic Quantum Electrodynamics, Adv. Theor. Math. Phys. 7, 667-710 (2003).

[MaSo 1$]$ A. Martinez and V. Sordoni. On the time-dependent Born-Oppenheimer approximation with smooth potential, Comptes Rendu Math. 334, 185-188 (2002).

[MaSo 2$]$ A. Martinez and V. Sordoni. Twisted Pseudodifferential Calculus and Application to the Quantum Evolution of Molecules, Mem. AMS 200, no. 936 (2009).

[Nen] G. Nenciu. Linear Adiabatic Theory, Exponential Estimates, Commun. Math. Phys. 152, 479-496 (1993).

[PST 1 G. Panati, H. Spohn and S. Teufel. Space-adiabatic perturbation theory, Adv. Theor. Math. Phys. 7, 145-204 (2003).

$\left[\mathrm{PST}_{2}\right]$ G. Panati, H. Spohn and S. Teufel. The Time-Dependent Born-Oppenheimer Approximation, ESIAM: Math. Model. and Num. Anal. 41, 297-314 (2007).

[ReSi 1 ] M. Reed and B. Simon. Methods of Modern Mathematical Physics. I: Functional Analysis, Academic Press (1978).

[ReSi 4 ] M. Reed and B. Simon. Methods of Modern Mathematical Physics. IV: Analysis of Operators, Academic Press (1978). 
[Si] B. Simon. Resonances and Complex Scaling: A Rigorous Overview, Int. J. Quant. Chem. 14, 529-542 (1978).

[SpTe] H. Spohn and S. Teufel. Adiabatic decoupling and time-dependent BornOppenheimer theory, Commun. Math. Phys. 224, 113-132 (2001).

[Ten] L. Tenuta. Quasi-static Limits in Nonrelativistic Quantum Electrodynamics, Ann. Henri Poincaré 9, 553-593 (2008).

[TeTe] L. Tenuta and S. Teufel. Effective dynamics for particles coupled to a quantized scalar field, Commun. Math. Phys. 280, 751-805 (2008).

[Teu $\left.{ }_{1}\right]$ S. Teufel. A Note on the Adiabatic Theorem Without Gap Condition, Lett. Math. Phys. 58, 261-266 (2001).

$\left[\mathrm{Teu}_{2}\right]$ S. Teufel. Adiabatic Perturbation Theory in Quantum Dynamics, Lecture Notes in Mathematics 1821, Springer (2003).

[WaTe] J. Wachsmuth and S. Teufel. Effective Hamiltonians for Constrained Quantum Systems, e-print arXiv:0907.0351v3 [math-ph] (2009).

[Zi] G.M. Zhislin. Discussion of the spectrum of the Schrödinger operator for systems of many particles, Tr. Mosk. Mat. Obs. 9, 81-128 (1960). 